\title{
Reaction of Polyhaloalkyl-Substituted Chromones, Pyrones, and Furanones with Salicylaldehydes as a Direct Route to Fused $2 \mathrm{H}$-Chromenes
}

\author{
Vyacheslav Ya. Sosnovskikh, ${ }^{* \dagger}$ Vladislav Yu. Korotaev, ${ }^{\dagger}$ Dmitry L. Chizhov, ${ }^{\ddagger}$ Igor B. Kutyashev, ${ }^{\dagger}$
} Danil S. Yachevskii, ${ }^{\ddagger}$ Olga N. Kazheva, ${ }^{\S}$ Oleg A. Dyachenko, ${ }^{\S}$ and Valery N. Charushin

${ }^{\dagger}$ Department of Chemistry, Ural State University, 620083 Ekaterinburg, Russian Federation, ${ }^{*}$ Institute of Organic Synthesis, Ural Branch of the Russian Academy of Sciences, 620219 Ekaterinburg, Russian Federation, and ${ }^{\S}$ Institute of Problems of Chemical Physics of the Russian Academy of Sciences, 142432 Chernogolovka, Russian Federation

\section{Table of contents}

1. X-ray crystal structures of compounds trans-6a and cis-6a

2. Table S1. Bond lengths $[\AA]$ and angles $\left[{ }^{\circ}\right]$ in compounds cis-6a and trans-6a

3. Table S2. Selected torsional angles $\left(^{\circ}\right)$

4. Table S3. The deviations of the atoms of the central pyrone ring from the least-squares plane $(\AA)$

5. Experimental Section

6. Copy of ${ }^{1} \mathrm{H}$ NMR spectrum of $\mathbf{2 c}$

7. Copy of ${ }^{19} \mathrm{~F}$ NMR spectrum of $\mathbf{2 c}$

8. Copy of ${ }^{1} \mathrm{H}$ NMR spectrum of $\mathbf{2 e}$

9. Copy of ${ }^{19} \mathrm{~F}$ NMR spectrum of $\mathbf{2 e}$

10. Copy of ${ }^{1} \mathrm{H}$ NMR spectrum of $\mathbf{2 h}$

11. Copy of ${ }^{1} \mathrm{H}$ NMR spectrum of $\mathbf{2 i}$

12. Copy of ${ }^{19}$ F NMR spectrum of $\mathbf{2} \mathbf{i}$

13. Copy of ${ }^{1} \mathrm{H}$ NMR spectrum of $\mathbf{2} \mathbf{j}$

14. Copy of ${ }^{19} \mathrm{~F}$ NMR spectrum of $\mathbf{2} \mathbf{j}$

15. Copy of ${ }^{1} \mathrm{H}$ NMR spectrum of $\mathbf{2 0}$

16. Copy of ${ }^{1} \mathrm{H}$ NMR spectrum of $\mathbf{2 r}$

17. Copy of ${ }^{19} \mathrm{~F}$ NMR spectrum of $\mathbf{2 r}$

18. Copy of ${ }^{1} \mathrm{H}$ NMR spectrum of $\mathbf{2 t}$

19. Copy of ${ }^{19} \mathrm{~F}$ NMR spectrum of $\mathbf{2 t}$

20. Copy of ${ }^{1} \mathrm{H}$ NMR spectrum of $\mathbf{2 x}$

21. Copy of ${ }^{1} \mathrm{H}$ NMR spectrum of $\mathbf{3 c}$

22. Copy of ${ }^{19} \mathrm{~F}$ NMR spectrum of $\mathbf{3 c}$

23. Copy of ${ }^{13} \mathrm{C}$ NMR spectrum of $3 c$

24. Copy of ${ }^{1} \mathrm{H}$ NMR spectrum of $\mathbf{3 d}$

25. Copy of ${ }^{19} \mathrm{~F}$ NMR spectrum of $\mathbf{3 d}$

26. Copy of ${ }^{1} \mathrm{H}$ NMR spectrum of $\mathbf{3 f}$

27. Copy of ${ }^{19}$ F NMR spectrum of $\mathbf{3 f}$

28. Copy of ${ }^{1} \mathrm{H}$ NMR spectrum of trans-6a

29. Copy of ${ }^{13} \mathrm{C}$ NMR spectrum of trans-6a 


\section{Trans-6a}

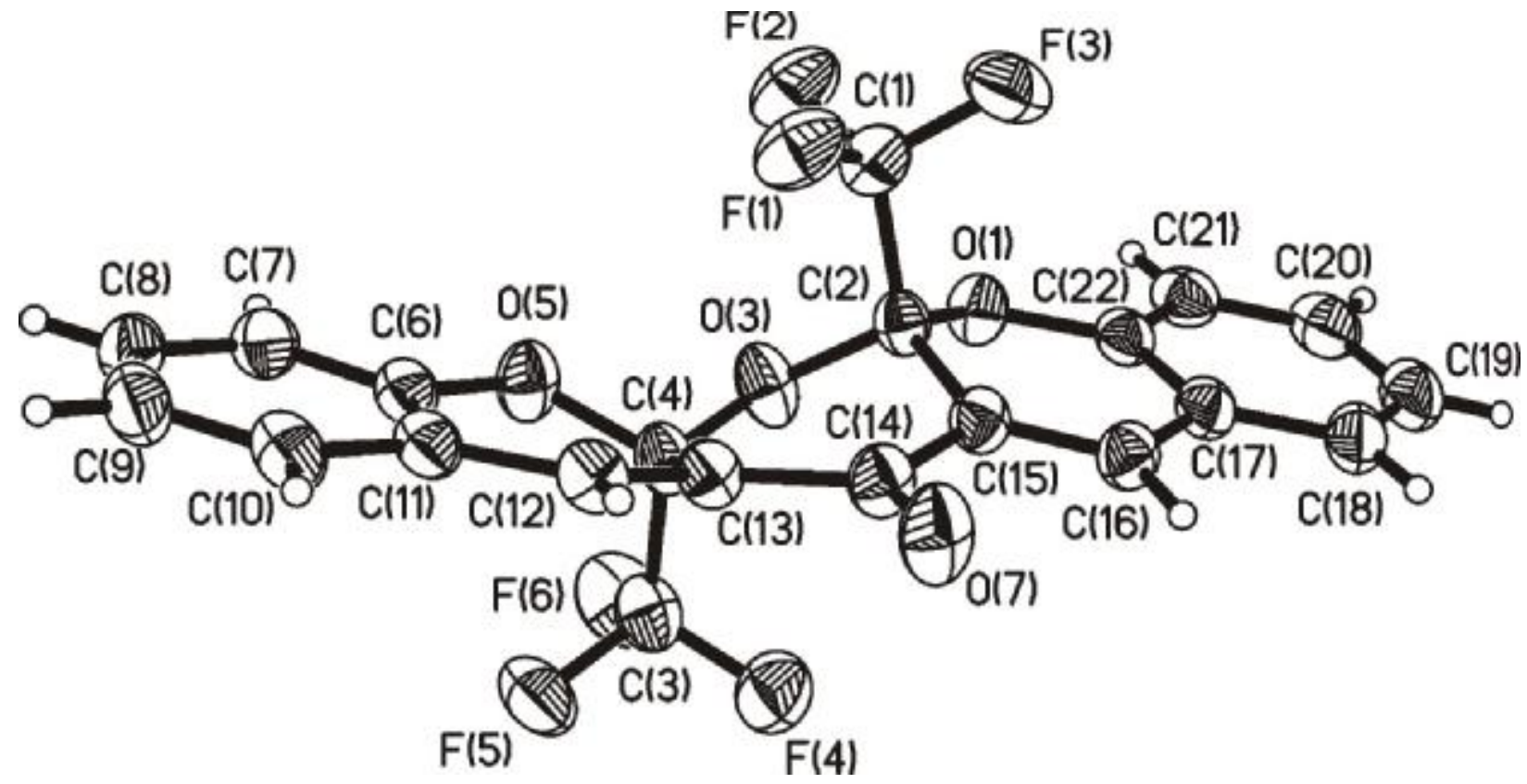

Cis-6a

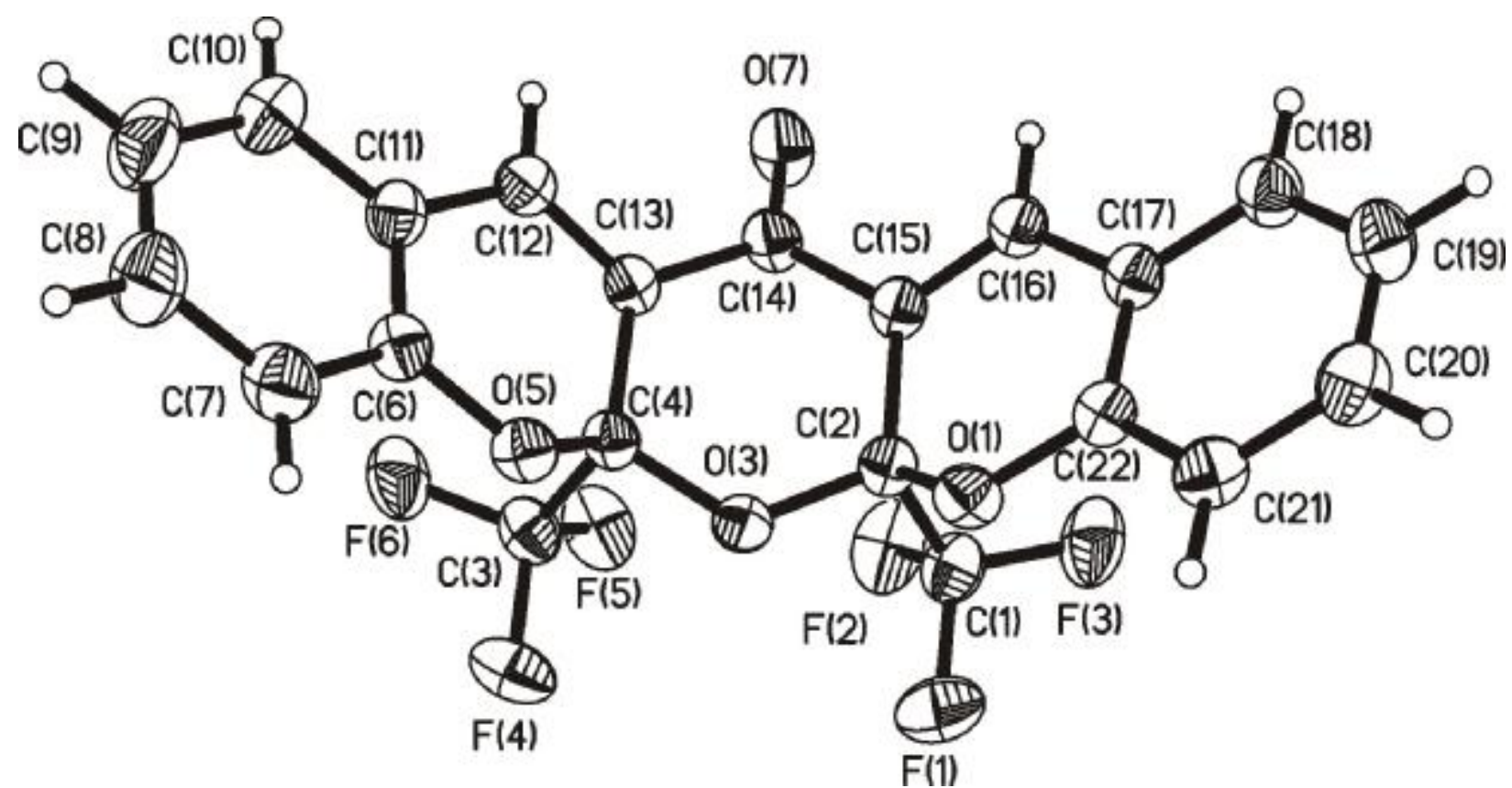


Trans-6a

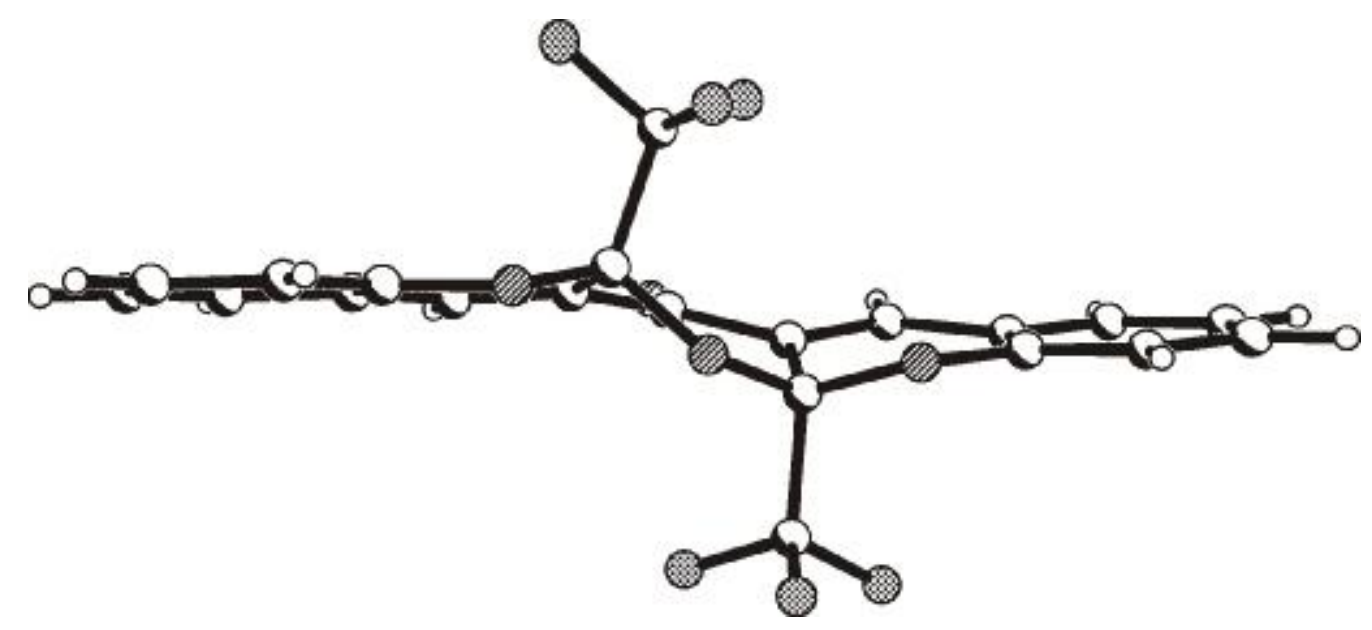

Cis-6a

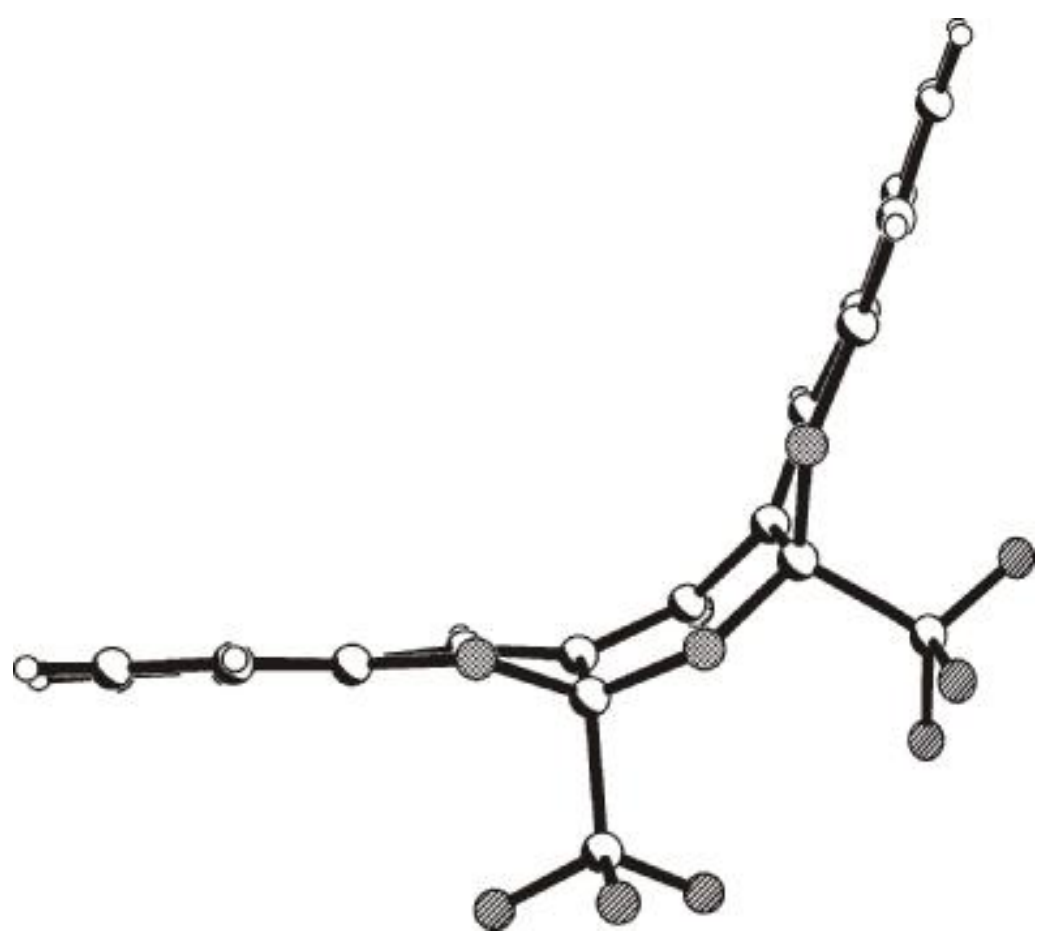


The X-ray studies were performed on a Enraf-Nonius CAD-4 diffractometer (for trans- and cis-6a) with $\omega / 2 \theta$ scanning and graphite monochromatic Mo-K $\alpha$ radiation. The crystal structures were solved by direct methods followed by Fourier synthesis with SHELXS-97 and refined by full-matrix least-squares methods for all non-hydrogen atoms with SHELXL-97 software packages [Sheldrick, G. M. SHELXS-97, SHELXL-97, Programs for Crystal Structure Determination and Refinement, University of Göttingen: Göttingen, Germany, 1997]. The coordinates of H atoms were found experimentally and refined in an isotropic approximation.

Crystallographic Data for trans-6a $\left(\mathrm{C}_{21} \mathrm{H}_{10} \mathrm{~F}_{6} \mathrm{O}_{4} ; M=440.29\right.$, CCDC no. 288999): triclinic, space group $P 1$ with $a=7.912(2), b=9.492(1), c=12.597(2) \AA$ 自 $\alpha=102.24(1), \beta=99.95(2), \gamma=$ 96.12(2) $)^{\circ}, V=900.5(3) \AA^{3} ; Z=2, D_{\text {calc }}=1.62 \mathrm{~g} \mathrm{~cm}^{-3} ; R=0.036\left[w R\left(F^{2}\right)=0.093\right]$ for 2706 reflections with $F_{0}>4 \sigma\left(F_{0}\right)$. The number of the parameters in the refinement was 291 .

Crystallographic Data for cis-6a $\left(\mathrm{C}_{21} \mathrm{H}_{10} \mathrm{~F}_{6} \mathrm{O}_{4} ; M=440.29\right.$, CCDC no. 289000): monoclinic,

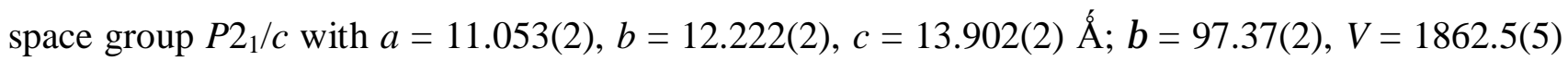
$\AA^{3} ; \mathrm{Z}=4, D_{\text {calc }}=1.57 \mathrm{~g} \mathrm{~cm}^{-3} ; R=0.034\left[w R\left(F^{2}\right)=0.093\right]$ for 2545 reflections with $F_{0}>4 \sigma\left(F_{0}\right)$. 
Table S1. Bond lengths $[\AA]$ and angles $\left[^{\circ}\right]$ in compounds cis-6a and trans-6a.

\begin{tabular}{|c|c|c|}
\hline Bond lengths $[\AA]$ & cis-6a & trans-6a \\
\hline $\mathrm{F}(1)-\mathrm{C}(1)$ & $1.327(2)$ & $1.326(2)$ \\
\hline $\mathrm{F}(2)-\mathrm{C}(1)$ & $1.326(2)$ & $1.327(2)$ \\
\hline $\mathrm{F}(3)-\mathrm{C}(1)$ & $1.332(2)$ & $1.331(2)$ \\
\hline $\mathrm{F}(4)-\mathrm{C}(3)$ & $1.328(2)$ & $1.333(2)$ \\
\hline $\mathrm{F}(5)-\mathrm{C}(3)$ & $1.319(2)$ & $1.311(2)$ \\
\hline $\mathrm{F}(6)-\mathrm{C}(3)$ & $1.335(2)$ & $1.335(2)$ \\
\hline $\mathrm{O}(1)-\mathrm{C}(22)$ & $1.388(2)$ & $1.386(2)$ \\
\hline $\mathrm{O}(1)-\mathrm{C}(2)$ & $1.410(2)$ & $1.402(2)$ \\
\hline $\mathrm{O}(3)-\mathrm{C}(4)$ & $1.405(2)$ & $1.409(2)$ \\
\hline $\mathrm{O}(3)-\mathrm{C}(2)$ & $1.409(2)$ & $1.414(2)$ \\
\hline $\mathrm{O}(5)-\mathrm{C}(6)$ & $1.389(2)$ & $1.384(2)$ \\
\hline $\mathrm{O}(5)-\mathrm{C}(4)$ & $1.415(2)$ & $1.402(2)$ \\
\hline $\mathrm{O}(7)-\mathrm{C}(14)$ & $1.208(2)$ & $1.220(2)$ \\
\hline $\mathrm{C}(1)-\mathrm{C}(2)$ & $1.538(2)$ & $1.527(2)$ \\
\hline $\mathrm{C}(2)-\mathrm{C}(15)$ & $1.499(2)$ & $1.504(2)$ \\
\hline $\mathrm{C}(3)-\mathrm{C}(4)$ & $1.547(2)$ & $1.534(2)$ \\
\hline $\mathrm{C}(4)-\mathrm{C}(13)$ & $1.499(2)$ & $1.507(2)$ \\
\hline $\mathrm{C}(6)-\mathrm{C}(7)$ & $1.376(2)$ & $1.371(2)$ \\
\hline$C(6)-C(11)$ & $1.387(2)$ & $1.396(2)$ \\
\hline $\mathrm{C}(7)-\mathrm{C}(8)$ & $1.378(3)$ & $1.383(3)$ \\
\hline $\mathrm{C}(8)-\mathrm{C}(9)$ & $1.375(3)$ & $1.369(3)$ \\
\hline $\mathrm{C}(9)-\mathrm{C}(10)$ & $1.374(3)$ & $1.373(3)$ \\
\hline $\mathrm{C}(10)-\mathrm{C}(11)$ & $1.396(3)$ & $1.397(2)$ \\
\hline $\mathrm{C}(11)-\mathrm{C}(12)$ & $1.443(2)$ & $1.438(2)$ \\
\hline $\mathrm{C}(12)-\mathrm{C}(13)$ & $1.335(2)$ & $1.337(2)$ \\
\hline $\mathrm{C}(13)-\mathrm{C}(14)$ & $1.475(2)$ & $1.473(2)$ \\
\hline $\mathrm{C}(14)-\mathrm{C}(15)$ & $1.473(2)$ & $1.473(2)$ \\
\hline $\mathrm{C}(15)-\mathrm{C}(16)$ & $1.343(2)$ & $1.332(2)$ \\
\hline $\mathrm{C}(16)-\mathrm{C}(17)$ & $1.437(2)$ & $1.441(2)$ \\
\hline $\mathrm{C}(17)-\mathrm{C}(22)$ & $1.394(2)$ & $1.383(2)$ \\
\hline $\mathrm{C}(17)-\mathrm{C}(18)$ & $1.398(2)$ & $1.400(2)$ \\
\hline $\mathrm{C}(18)-\mathrm{C}(19)$ & $1.375(2)$ & $1.375(3)$ \\
\hline $\mathrm{C}(19)-\mathrm{C}(20)$ & $1.378(3)$ & $1.381(3)$ \\
\hline $\mathrm{C}(20)-\mathrm{C}(21)$ & $1.378(3)$ & $1.378(3)$ \\
\hline $\mathrm{C}(21)-\mathrm{C}(22)$ & $1.376(2)$ & $1.378(2)$ \\
\hline \multicolumn{3}{|l|}{ Angles $\left[{ }^{\circ}\right]$} \\
\hline $\mathrm{C}(22)-\mathrm{O}(1)-\mathrm{C}(2)$ & $119.3(1)$ & $120.6(1)$ \\
\hline $\mathrm{C}(4)-\mathrm{O}(3)-\mathrm{C}(2)$ & $124.6(1)$ & $121.4(1)$ \\
\hline $\mathrm{C}(6)-\mathrm{O}(5)-\mathrm{C}(4)$ & $118.0(1)$ & $119.8(1)$ \\
\hline $\mathrm{F}(2)-\mathrm{C}(1)-\mathrm{F}(1)$ & $107.6(2)$ & $107.5(1)$ \\
\hline $\mathrm{F}(2)-\mathrm{C}(1)-\mathrm{F}(3)$ & $107.2(1)$ & $108.0(1)$ \\
\hline $\mathrm{F}(1)-\mathrm{C}(1)-\mathrm{F}(3)$ & $107.4(1)$ & $107.6(1)$ \\
\hline $\mathrm{F}(2)-\mathrm{C}(1)-\mathrm{C}(2)$ & $110.8(1)$ & $111.7(1)$ \\
\hline $\mathrm{F}(1)-\mathrm{C}(1)-\mathrm{C}(2)$ & $112.2(1)$ & $112.1(1)$ \\
\hline $\mathrm{F}(3)-\mathrm{C}(1)-\mathrm{C}(2)$ & $111.4(1)$ & $109.7(1)$ \\
\hline $\mathrm{O}(3)-\mathrm{C}(2)-\mathrm{O}(1)$ & $104.9(1)$ & $101.6(1)$ \\
\hline
\end{tabular}




\begin{tabular}{|c|c|c|}
\hline $\mathrm{O}(3)-\mathrm{C}(2)-\mathrm{C}(15)$ & $116.5(1)$ & $112.3(1)$ \\
\hline $\mathrm{O}(1)-\mathrm{C}(2)-\mathrm{C}(15)$ & $113.4(1)$ & $115.4(1)$ \\
\hline $\mathrm{O}(3)-\mathrm{C}(2)-\mathrm{C}(1)$ & $103.5(1)$ & $109.3(1)$ \\
\hline $\mathrm{O}(1)-\mathrm{C}(2)-\mathrm{C}(1)$ & $106.5(1)$ & $105.1(1)$ \\
\hline $\mathrm{C}(15)-\mathrm{C}(2)-\mathrm{C}(1)$ & $111.0(1)$ & $112.3(1)$ \\
\hline $\mathrm{F}(5)-\mathrm{C}(3)-\mathrm{F}(4)$ & $107.5(1)$ & $107.6(1)$ \\
\hline $\mathrm{F}(5)-\mathrm{C}(3)-\mathrm{F}(6)$ & $107.2(1)$ & $107.9(1)$ \\
\hline $\mathrm{F}(4)-\mathrm{C}(3)-\mathrm{F}(6)$ & $106.9(1)$ & $106.8(1)$ \\
\hline $\mathrm{F}(5)-\mathrm{C}(3)-\mathrm{C}(4)$ & $111.6(1)$ & $112.8(1)$ \\
\hline $\mathrm{F}(4)-\mathrm{C}(3)-\mathrm{C}(4)$ & $111.9(1)$ & $110.6(1)$ \\
\hline $\mathrm{F}(6)-\mathrm{C}(3)-\mathrm{C}(4)$ & $111.4(1)$ & $110.9(1)$ \\
\hline $\mathrm{O}(3)-\mathrm{C}(4)-\mathrm{O}(5)$ & $104.9(1)$ & $104.6(1)$ \\
\hline $\mathrm{O}(3)-\mathrm{C}(4)-\mathrm{C}(13)$ & $116.6(1)$ & $115.7(1)$ \\
\hline $\mathrm{O}(5)-\mathrm{C}(4)-\mathrm{C}(13)$ & $113.3(1)$ & $113.6(1)$ \\
\hline $\mathrm{O}(3)-\mathrm{C}(4)-\mathrm{C}(3)$ & $103.7(1)$ & $103.6(1)$ \\
\hline $\mathrm{O}(5)-\mathrm{C}(4)-\mathrm{C}(3)$ & $106.8(1)$ & $107.6(1)$ \\
\hline $\mathrm{C}(13)-\mathrm{C}(4)-\mathrm{C}(3)$ & $110.7(1)$ & $110.9(1)$ \\
\hline $\mathrm{C}(7)-\mathrm{C}(6)-\mathrm{C}(11)$ & $121.8(2)$ & $121.7(2)$ \\
\hline $\mathrm{C}(7)-\mathrm{C}(6)-\mathrm{O}(5)$ & $117.0(1)$ & $117.5(1)$ \\
\hline $\mathrm{C}(11)-\mathrm{C}(6)-\mathrm{O}(5)$ & $121.1(1)$ & $120.7(1)$ \\
\hline $\mathrm{C}(6)-\mathrm{C}(7)-\mathrm{C}(8)$ & $118.9(2)$ & $118.7(2)$ \\
\hline $\mathrm{C}(9)-\mathrm{C}(8)-\mathrm{C}(7)$ & $120.7(2)$ & $121.0(2)$ \\
\hline $\mathrm{C}(10)-\mathrm{C}(9)-\mathrm{C}(8)$ & $120.0(2)$ & $120.1(2)$ \\
\hline $\mathrm{C}(9)-\mathrm{C}(10)-\mathrm{C}(11)$ & $120.7(2)$ & $120.6(2)$ \\
\hline $\mathrm{C}(6)-\mathrm{C}(11)-\mathrm{C}(10)$ & $117.9(2)$ & $117.8(1)$ \\
\hline $\mathrm{C}(6)-\mathrm{C}(11)-\mathrm{C}(12)$ & $117.4(1)$ & $117.5(1)$ \\
\hline $\mathrm{C}(10)-\mathrm{C}(11)-\mathrm{C}(12)$ & $124.4(2)$ & $124.5(1)$ \\
\hline $\mathrm{C}(13)-\mathrm{C}(12)-\mathrm{C}(11)$ & $121.6(1)$ & $121.9(1)$ \\
\hline $\mathrm{C}(12)-\mathrm{C}(13)-\mathrm{C}(14)$ & $120.8(1)$ & $123.1(1)$ \\
\hline $\mathrm{C}(12)-\mathrm{C}(13)-\mathrm{C}(4)$ & $118.1(1)$ & $118.3(1)$ \\
\hline $\mathrm{C}(14)-\mathrm{C}(13)-\mathrm{C}(4)$ & $120.2(1)$ & $118.5(1)$ \\
\hline $\mathrm{O}(7)-\mathrm{C}(14)-\mathrm{C}(15)$ & $124.3(1)$ & $123.0(1)$ \\
\hline $\mathrm{O}(7)-\mathrm{C}(14)-\mathrm{C}(13)$ & $123.2(1)$ & $121.7(1)$ \\
\hline $\mathrm{C}(15)-\mathrm{C}(14)-\mathrm{C}(13)$ & $111.4(1)$ & $115.2(1)$ \\
\hline $\mathrm{C}(16)-\mathrm{C}(15)-\mathrm{C}(14)$ & $120.4(1)$ & $123.5(1)$ \\
\hline $\mathrm{C}(16)-\mathrm{C}(15)-\mathrm{C}(2)$ & $119.1(1)$ & $120.0(1)$ \\
\hline $\mathrm{C}(14)-\mathrm{C}(15)-\mathrm{C}(2)$ & $119.8(1)$ & $116.5(1)$ \\
\hline $\mathrm{C}(15)-\mathrm{C}(16)-\mathrm{C}(17)$ & $121.4(1)$ & $121.5(1)$ \\
\hline $\mathrm{C}(22)-\mathrm{C}(17)-\mathrm{C}(18)$ & $118.4(1)$ & $118.2(2)$ \\
\hline $\mathrm{C}(22)-\mathrm{C}(17)-\mathrm{C}(16)$ & $117.6(1)$ & $118.2(1)$ \\
\hline $\mathrm{C}(18)-\mathrm{C}(17)-\mathrm{C}(16)$ & $123.6(1)$ & $123.5(2)$ \\
\hline $\mathrm{C}(19)-\mathrm{C}(18)-\mathrm{C}(17)$ & $120.3(2)$ & $120.1(2)$ \\
\hline $\mathrm{C}(18)-\mathrm{C}(19)-\mathrm{C}(20)$ & $119.7(2)$ & $120.1(2)$ \\
\hline $\mathrm{C}(21)-\mathrm{C}(21)-\mathrm{C}(21)-\mathrm{C}(22)-\mathrm{O}(1)$ & $121.5(2)$ & $121.0(2)$ \\
\hline $\mathrm{O}(1)-\mathrm{C}(22)-\mathrm{C}(17)$ & $117.6(2)$ & $118.3(2)$ \\
\hline & $121.5(1)$ & $116.2(1)$ \\
\hline
\end{tabular}


Table S2. Selected torsional angles $\left(^{\circ}\right)$

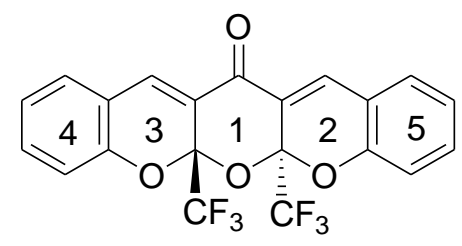

trans-6a

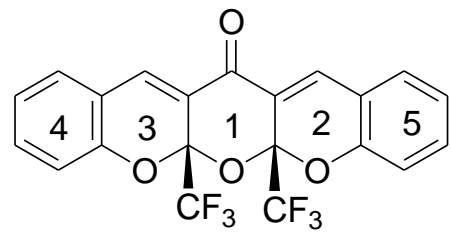

cis-6a

\begin{tabular}{|c|c|c|}
\hline Cycles & cis-6a & trans-6a \\
\hline $1-2$ & 141.4 & 152.3 \\
\hline $1-3$ & 142.0 & 150.2 \\
\hline $1-4$ & 144.9 & 153.7 \\
\hline $1-5$ & 142.5 & 154.6 \\
\hline $2-3$ & 104.9 & 165.8 \\
\hline $2-4$ & 109.3 & 170.9 \\
\hline $2-5$ & 176.4 & 177.8 \\
\hline $3-4$ & 173.3 & 174.3 \\
\hline $3-5$ & 106.8 & 165.5 \\
\hline $4-5$ & 111.5 & 171.1 \\
\hline
\end{tabular}

Table S3. The deviations of the atoms of the central pyrone ring from the least-squares plane (Á)

\begin{tabular}{|c|c|c|}
\hline Atoms & cis-6a & trans-6a \\
\hline $\mathrm{O}(3)$ & -0.08 & 0.11 \\
\hline $\mathrm{C}(2)$ & -0.005 & -0.29 \\
\hline $\mathrm{C}(4)$ & 0.02 & 0.15 \\
\hline $\mathrm{C}(13)$ & 0.13 & -0.22 \\
\hline $\mathrm{C}(14)$ & -0.21 & 0.04 \\
\hline $\mathrm{C}(15)$ & 0.15 & 0.21 \\
\hline $\mathrm{O}(7)$ & -0.55 & 0.09 \\
\hline
\end{tabular}




\section{Experimental Section}

${ }^{1} \mathrm{H},{ }^{19} \mathrm{~F}$ and ${ }^{13} \mathrm{C}$ NMR spectra were recorded at 400, 376 and $100 \mathrm{MHz}$, respectively; chemical shifts are given in $\delta \mathrm{ppm}$ downfield from tetramethylsilane and hexafluorobenzene as internal standards. IR spectra were measured in $\mathrm{KBr}$ or Nujol. Elemental analyses were performed on a microanalyzer. Melting points are uncorrected. The starting polyhaloalkyl-containing chromones, pyrones and furanones were prepared according to the described procedure. ${ }^{27-29}$

9-Chloro-5a-(difluoromethyl)-5a H,11H-chromeno[2,3-b]chromen-11-one (2b): mp 143-144 ${ }^{\circ} \mathrm{C}$; IR (KBr) 1671, 1617, 1602, 1566, 1466, $1423 \mathrm{~cm}^{-1} ;{ }^{1} \mathrm{H}$ NMR $\left(400 \mathrm{MHz}, \mathrm{CDCl}_{3}\right) \delta 5.77(\mathrm{t}, 1 \mathrm{H}, J$ $=54.7 \mathrm{~Hz}), 7.06-7.12(\mathrm{~m}, 3 \mathrm{H}), 7.37-7.45(\mathrm{~m}, 2 \mathrm{H}), 7.51(\mathrm{dd}, 1 \mathrm{H}, J=8.8,2.7 \mathrm{~Hz}), 7.92(\mathrm{~s}, 1 \mathrm{H}), 7.95$ $(\mathrm{d}, 1 \mathrm{H}, J=2.7 \mathrm{~Hz}) ;{ }^{19} \mathrm{~F}$ NMR $\left(376 \mathrm{MHz}, \mathrm{CDCl}_{3} / \mathrm{C}_{6} \mathrm{~F}_{6}\right) \delta 27.19(\mathrm{dd}, 1 \mathrm{~F}, J=291.5,54.9 \mathrm{~Hz}), 29.38$ (dd, $1 \mathrm{~F}, J=291.5,54.6 \mathrm{~Hz}$ ). Anal. Calcd for $\mathrm{C}_{17} \mathrm{H}_{9} \mathrm{ClF}_{2} \mathrm{O}_{3}: \mathrm{C}, 61.01 ; \mathrm{H}, 2.71$. Found: C, 60.93; H, 2.88 .

5a-(Difluoromethyl)-5aH,11H-chromeno[2,3-b]chromen-11-one (2c): mp $126-127 \quad{ }^{\circ} \mathrm{C}$; IR $(\mathrm{KBr}) 1666,1610,1569,1461 \mathrm{~cm}^{-1} ;{ }^{1} \mathrm{H} \mathrm{NMR}\left(400 \mathrm{MHz}, \mathrm{CDCl}_{3}\right) \delta 5.78(\mathrm{t}, 1 \mathrm{H}, J=54.7 \mathrm{~Hz})$, 7.06-7.17 (m, 4H), 7.37-7.43 (m, 2H), 7.58 (ddd, 1H, J = 8.4, 7.3, $1.7 \mathrm{~Hz}), 7.90(\mathrm{~s}, 1 \mathrm{H}), 8.00$ (dd, $1 \mathrm{H}, J=7.8,1.7 \mathrm{~Hz}) ;{ }^{19} \mathrm{~F} \mathrm{NMR}\left(376 \mathrm{MHz}, \mathrm{CDCl}_{3} / \mathrm{C}_{6} \mathrm{~F}_{6}\right) \delta 27.11(\mathrm{dd}, 1 \mathrm{~F}, J=290.4,54.8 \mathrm{~Hz}), 29.07$ (dd, $1 \mathrm{~F}, J=290.4,54.7 \mathrm{~Hz}$ ). Anal. Calcd for $\mathrm{C}_{17} \mathrm{H}_{10} \mathrm{~F}_{2} \mathrm{O}_{3}$ : C, 68.00; H, 3.36. Found: C, 68.07; H, 3.34.

5a-(Difluoromethyl)-9-methyl-5a $H, 11 H$-chromeno[2,3-b]chromen-11-one (2d): mp 132-133 ${ }^{\circ} \mathrm{C}$; IR (KBr) 1673, 1611, 1569, 1488, 1459, $1422 \mathrm{~cm}^{-1} ;{ }^{1} \mathrm{H}$ NMR (400 MHz, $\left.\mathrm{CDCl}_{3}\right) \delta 2.35$ (s, 3H), $5.77(\mathrm{t}, 1 \mathrm{H} J=54.7 \mathrm{~Hz}), 7.00-7.10(\mathrm{~m}, 3 \mathrm{H}), 7.36-7.43(\mathrm{~m}, 3 \mathrm{H}), 7.79$ (br.d, 1H, $J=1.7 \mathrm{~Hz}), 7.90$ (s, $1 \mathrm{H}) ;{ }^{19} \mathrm{~F}$ NMR $\left(376 \mathrm{MHz}, \mathrm{CDCl}_{3} / \mathrm{C}_{6} \mathrm{~F}_{6}\right) \delta 27.16(\mathrm{dd}, 1 \mathrm{~F}, J=290.1,54.8 \mathrm{~Hz}), 29.08(\mathrm{dd}, 1 \mathrm{~F}, J=$ 290.1, 54.7 Hz). Anal. Calcd for $\mathrm{C}_{18} \mathrm{H}_{12} \mathrm{~F}_{2} \mathrm{O}_{3}$ : C, 68.79; H, 3.85. Found: C, 68.60; H, 3.86.

2-Bromo-5a-(difluoromethyl)-9-nitro-5a $H, 11 H$-chromeno[2,3-b]chromen-11-one $\quad(2 \mathrm{e}): \quad \mathrm{mp}$ 201-202 ${ }^{\circ} \mathrm{C}$; IR (KBr) 1677, 1618, 1589, 1557, 1534, 1472, 1442, $1337 \mathrm{~cm}^{-1} ;{ }^{1} \mathrm{H}$ NMR (400 MHz, 
$\left.\mathrm{CDCl}_{3}\right) \delta 5.81(\mathrm{t}, 1 \mathrm{H}, J=54.7 \mathrm{~Hz}), 7.00-7.03(\mathrm{~m}, 1 \mathrm{H}), 7.27(\mathrm{~d}, 1 \mathrm{H}, J=9.1 \mathrm{~Hz}), 7.53-7.57(\mathrm{~m}, 2 \mathrm{H})$, $7.90(\mathrm{~s}, 1 \mathrm{H}), 8.44(\mathrm{dd}, 1 \mathrm{H}, J=9.1,2.8 \mathrm{~Hz}), 8.89(\mathrm{~d}, 1 \mathrm{H}, J=2.8 \mathrm{~Hz}) ;{ }^{19} \mathrm{~F}$ NMR $(376 \mathrm{MHz}$, $\left.\mathrm{CDCl}_{3} / \mathrm{C}_{6} \mathrm{~F}_{6}\right) \delta 27.29(\mathrm{dd}, 1 \mathrm{~F}, J=294.2,54.9 \mathrm{~Hz}), 29.84(\mathrm{dd}, 1 \mathrm{~F}, J=294.2,54.5 \mathrm{~Hz})$. Anal. Calcd for $\mathrm{C}_{17} \mathrm{H}_{8} \mathrm{BrF}_{2} \mathrm{NO}_{5}: \mathrm{C}, 48.14 ; \mathrm{H}, 1.90 ; \mathrm{N}, 3.30$. Found: C, 48.11; H, 1.97; N, 3.46.

2-Bromo-9-chloro-5a-(difluoromethyl)-5aH,11H-chromeno[2,3-b]chromen-11-one (2f): $\mathrm{mp}$ 159-160 ${ }^{\circ} \mathrm{C}$; IR (KBr) 1677, 1620, 1601, 1560, 1465, $1430 \mathrm{~cm}^{-1} ;{ }^{1} \mathrm{H}$ NMR (400 MHz, $\left.\mathrm{CDCl}_{3}\right) \delta 5.77$ $(\mathrm{t}, 1 \mathrm{H}, J=54.7 \mathrm{~Hz}), 6.97-7.00(\mathrm{~m}, 1 \mathrm{H}), 7.08(\mathrm{~d}, 1 \mathrm{H}, J=8.8 \mathrm{~Hz}), 7.48-7.54(\mathrm{~m}, 3 \mathrm{H}), 7.83(\mathrm{~s}, 1 \mathrm{H})$, $7.95(\mathrm{~d}, 1 \mathrm{H}, J=2.6 \mathrm{~Hz}) ;{ }^{19} \mathrm{~F} \mathrm{NMR}\left(376 \mathrm{MHz}, \mathrm{CDCl}_{3} / \mathrm{C}_{6} \mathrm{~F}_{6}\right) \delta 27.54(\mathrm{dd}, 1 \mathrm{~F}, J=292.7,54.8 \mathrm{~Hz})$, 29.70 (dd, $1 \mathrm{~F}, J=292.7,54.7 \mathrm{~Hz}$ ). Anal. Calcd for $\mathrm{C}_{17} \mathrm{H}_{8} \mathrm{BrClF}_{2} \mathrm{O}_{3}: \mathrm{C}, 49.37 ; \mathrm{H}, 1.95$. Found: C, 49.38; H, 1.74 .

9-Chloro-5a-(difluoromethyl)-2-methoxy-5a $H, 11 H$-chromeno[2,3-b]chromen-11-one (2g): mp $174-175{ }^{\circ} \mathrm{C}$; IR (KBr) 1672, 1623, 1573, 1489, 1468, $1420 \mathrm{~cm}^{-1} ;{ }^{1} \mathrm{H}$ NMR (400 MHz, $\left.\mathrm{CDCl}_{3}\right) \delta 3.81$ (s, 3H), $5.75(\mathrm{t}, 1 \mathrm{H}, J=54.7 \mathrm{~Hz}), 6.87(\mathrm{~d}, 1 \mathrm{H}, J=2.8 \mathrm{~Hz}), 6.98(\mathrm{dd}, 1 \mathrm{H}, J=8.9,2.8 \mathrm{~Hz}), 7.02(\mathrm{~d}$, $1 \mathrm{H}, J=8.9 \mathrm{~Hz}), 7.07(\mathrm{~d}, 1 \mathrm{H}, J=8.8 \mathrm{~Hz}), 7.51(\mathrm{dd}, 1 \mathrm{H}, J=8.8,2.7 \mathrm{~Hz}), 7.88(\mathrm{~s}, 1 \mathrm{H}), 7.95(\mathrm{~d}, 1 \mathrm{H}, J$ $=2.7 \mathrm{~Hz}) ;{ }^{19} \mathrm{~F}$ NMR $\left(376 \mathrm{MHz}, \mathrm{CDCl}_{3} / \mathrm{C}_{6} \mathrm{~F}_{6}\right) \delta 27.29(\mathrm{dd}, 1 \mathrm{~F}, J=291.4,54.9 \mathrm{~Hz}), 29.38(\mathrm{dd}, 1 \mathrm{~F}, J=$ 291.4, 54.5 Hz). Anal. Calcd for $\mathrm{C}_{18} \mathrm{H}_{11} \mathrm{ClF}_{2} \mathrm{O}_{4}$ : C, 59.28; H, 3.04. Found: C, 59.21; H, 3.00.

5a-(Difluoromethyl)-2-methoxy-9-methyl-5a $H, 11 H$-chromeno[2,3-b]chromen-11-one (2h): mp 164-165 ${ }^{\circ} \mathrm{C}$; IR (KBr) 1672, 1619, 1574, 1489, 1453, $1422 \mathrm{~cm}^{-1} ;{ }^{1} \mathrm{H}$ NMR (400 MHz, $\left.\mathrm{CDCl}_{3}\right) \delta 2.35$ (s, 3H), $3.81(\mathrm{~s}, 3 \mathrm{H}), 5.75(\mathrm{t}, 1 \mathrm{H}, J=54.7 \mathrm{~Hz}), 6.86(\mathrm{~d}, 1 \mathrm{H}, J=2.9 \mathrm{~Hz}), 6.96(\mathrm{dd}, 1 \mathrm{H}, J=8.9,2.9$ Hz), $7.01(\mathrm{~d}, 1 \mathrm{H}, J=8.4 \mathrm{~Hz}), 7.02(\mathrm{~d}, 1 \mathrm{H}, J=8.9 \mathrm{~Hz}), 7.38$ (br.dd, 1H, $J=8.4,2.0 \mathrm{~Hz}), 7.78$ (br.d, $1 \mathrm{H}, J=2.0 \mathrm{~Hz}), 7.85(\mathrm{~s}, 1 \mathrm{H}) ;{ }^{19} \mathrm{~F}$ NMR $\left(376 \mathrm{MHz}, \mathrm{CDCl}_{3} / \mathrm{C}_{6} \mathrm{~F}_{6}\right) \delta 27.27(\mathrm{dd}, 1 \mathrm{~F}, J=290.1,54.8$ $\mathrm{Hz}$ ), 29.10 (dd, $1 \mathrm{~F}, J=290.1,54.6 \mathrm{~Hz}$ ). Anal. Calcd for $\mathrm{C}_{19} \mathrm{H}_{14} \mathrm{~F}_{2} \mathrm{O}_{4}$ : C, 66.28; H, 4.10. Found: C, 66.30; H, 3.96.

9-Nitro-5a-(trifluoromethyl)-5a $\boldsymbol{H , 1 1 H}$-chromeno[2,3-b]chromen-11-one (2i): $\mathrm{mp}$ 185-186 ${ }^{\circ} \mathrm{C}$; IR (KBr) 1683, 1622, 1606, 1592, 1569, 1527, 1472, 1440, $1351 \mathrm{~cm}^{-1} ;{ }^{1} \mathrm{H}$ NMR (400 MHz, CDCl $)$ 
$\delta 7.15-7.20(\mathrm{~m}, 2 \mathrm{H}), 7.32(\mathrm{~d}, 1 \mathrm{H}, J=9.1 \mathrm{~Hz}), 7.45-7.54(\mathrm{~m}, 2 \mathrm{H}), 8.05(\mathrm{~s}, 1 \mathrm{H}), 8.46(\mathrm{dd}, 1 \mathrm{H}, J=9.1$,

$2.8 \mathrm{~Hz}), 8.91(\mathrm{~d}, 1 \mathrm{H}, J=2.8 \mathrm{~Hz}) ;{ }^{19} \mathrm{~F} \mathrm{NMR}\left(376 \mathrm{MHz}, \mathrm{CDCl}_{3} / \mathrm{C}_{6} \mathrm{~F}_{6}\right) \delta 75.82$ (s). Anal. Calcd for $\mathrm{C}_{17} \mathrm{H}_{8} \mathrm{~F}_{3} \mathrm{NO}_{5}$ : C, 56.21; H, 2.22; N, 3.86. Found: C, 56.27; H, 2.44; N, 3.88.

9-Methyl-5a-(trifluoromethyl)-5a $\boldsymbol{H , 1 1 H}$-chromeno[2,3-b]chromen-11-one (2k): mp 120-121 ${ }^{\circ} \mathrm{C}$; IR (KBr) 1678, 1624, 1608, 1570, 1487, 1459, $1423 \mathrm{~cm}^{-1} ;{ }^{1} \mathrm{H}$ NMR (400 MHz, $\left.\mathrm{CDCl}_{3}\right) \delta 2.37$ (s, 3H), 7.05-7.16 (m, 3H), 7.39-7.47 (m, 3H), 7.80 (br.d, 1H, J=1.8 Hz), 7.95 (s, 1H); ${ }^{19} \mathrm{~F}$ NMR (376 $\left.\mathrm{MHz}, \mathrm{CDCl}_{3} / \mathrm{C}_{6} \mathrm{~F}_{6}\right) \delta 76.08$ (s). Anal. Calcd for $\mathrm{C}_{18} \mathrm{H}_{11} \mathrm{~F}_{3} \mathrm{O}_{3}$ : C, 65.07; H, 3.34. Found: C, 64.95; H, 3.39.

2-Bromo-9-nitro-5a-(trifluoromethyl)-5a $H, 11 H$-chromeno[2,3-b]chromen-11-one $\quad(2 \mathrm{l}): \quad \mathrm{mp}$ 213-214 ${ }^{\circ} \mathrm{C}$; IR (KBr) 1677, 1625, 1589, 1560, 1534, 1472, 1443, $1351 \mathrm{~cm}^{-1} ;{ }^{1} \mathrm{H}$ NMR (400 MHz, $\left.\mathrm{CDCl}_{3}\right) \delta 7.06-7.10(\mathrm{~m}, 1 \mathrm{H}), 7.33(\mathrm{~d}, 1 \mathrm{H}, J=9.1 \mathrm{~Hz}), 7.58-7.63(\mathrm{~m}, 2 \mathrm{H}), 7.97(\mathrm{~s}, 1 \mathrm{H}), 8.48(\mathrm{dd}, 1 \mathrm{H}$, $J=9.1,2.8 \mathrm{~Hz}), 8.90(\mathrm{~d}, 1 \mathrm{H}, J=2.8 \mathrm{~Hz}) ;{ }^{19} \mathrm{~F} \mathrm{NMR}\left(376 \mathrm{MHz}, \mathrm{CDCl}_{3} / \mathrm{C}_{6} \mathrm{~F}_{6}\right) \delta 75.99$ (s). Anal. Calcd for $\mathrm{C}_{17} \mathrm{H}_{7} \mathrm{BrF}_{3} \mathrm{NO}_{5}$ : C, 46.18; H, 1.60; N, 3.17. Found: C, 46.20; H, 1.42; N, 3.04.

2-Bromo-5a-(trifluoromethyl)-5aH,11H-chromeno[2,3-b]chromen-11-one (2m): mp 196-197 ${ }^{\circ} \mathrm{C}$; IR (KBr) 1673, 1615, 1558, $1462 \mathrm{~cm}^{-1} ;{ }^{1} \mathrm{H}$ NMR (400 MHz, $\left.\mathrm{CDCl}_{3}\right) \delta$ 7.03-7.07 (m, 1H), 7.15$7.22(\mathrm{~m}, 2 \mathrm{H}), 7.52-7.57(\mathrm{~m}, 2 \mathrm{H}), 7.63(\mathrm{ddd}, 1 \mathrm{H}, J=8.5,7.0,1.6 \mathrm{~Hz}), 7.88(\mathrm{~s}, 1 \mathrm{H}), 8.02(\mathrm{dd}, 1 \mathrm{H}, J=$ 7.8, $1.6 \mathrm{~Hz}) ;{ }^{19} \mathrm{~F}$ NMR $\left(376 \mathrm{MHz}, \mathrm{CDCl}_{3} / \mathrm{C}_{6} \mathrm{~F}_{6}\right) \delta 76.12$ (s). Anal. Calcd for $\mathrm{C}_{17} \mathrm{H}_{8} \mathrm{BrF}_{3} \mathrm{O}_{3}$ : C, 51.41; H, 2.03. Found: C, 51.42; H, 2.12.

2-Bromo-9-methyl-5a-(trifluoromethyl)-5aH,11H-chromeno[2,3-b]chromen-11-one (2n): mp 198-199 ${ }^{\circ} \mathrm{C}$; IR (KBr) 1675, 1625, 1561, 1488, 1475, $1428 \mathrm{~cm}^{-1} ;{ }^{1} \mathrm{H}$ NMR (400 MHz, $\left.\mathrm{CDCl}_{3}\right) \delta 2.37$ (s, 3H), 7.03-7.07 (m, 2H), 7.41-7.53 (m, 3H), 7.80 (br.s, 1H), 7.86 (s, 1H). ${ }^{19} \mathrm{~F} \mathrm{NMR} \mathrm{(376} \mathrm{MHz,}$ $\left.\mathrm{CDCl}_{3} / \mathrm{C}_{6} \mathrm{~F}_{6}\right) \delta 76.27$ (s). Anal. Calcd for $\mathrm{C}_{18} \mathrm{H}_{10} \mathrm{BrF}_{3} \mathrm{O}_{3}$ : C, 52.58; H, 2.45. Found: C, 52.53; H, 2.26. 2-Methoxy-9-nitro-5a-(trifluoromethyl)-5a $H, 11 H$-chromeno[2,3-b]chromen-11-one (2o): mp $181-182{ }^{\circ} \mathrm{C}$; IR (KBr) 1674, 1623, 1575, 1527, 1490, 1471, 1440, $1347 \mathrm{~cm}^{-1} ;{ }^{1} \mathrm{H}$ NMR (400 MHz, $\left.\mathrm{CDCl}_{3}\right) \delta 3.83(\mathrm{~s}, 3 \mathrm{H}), 6.93(\mathrm{~d}, 1 \mathrm{H}, J=2.8 \mathrm{~Hz}), 7.06(\mathrm{dd}, 1 \mathrm{H}, J=9.0,2.8 \mathrm{~Hz}), 7.11(\mathrm{~d}, 1 \mathrm{H}, J=9.0$ 
Hz), $7.32(\mathrm{~d}, 1 \mathrm{H}, J=9.1 \mathrm{~Hz}), 8.01(\mathrm{~s}, 1 \mathrm{H}), 8.46(\mathrm{dd}, 1 \mathrm{H}, J=9.1,2.8 \mathrm{~Hz}), 8.90(\mathrm{~d}, 1 \mathrm{H}, J=2.8 \mathrm{~Hz})$;

${ }^{19} \mathrm{~F}$ NMR $\left(376 \mathrm{MHz}, \mathrm{CDCl}_{3} / \mathrm{C}_{6} \mathrm{~F}_{6}\right) \delta 75.88$ (s). Anal. Calcd for $\mathrm{C}_{18} \mathrm{H}_{10} \mathrm{~F}_{3} \mathrm{NO}_{6}: \mathrm{C}, 54.97 ; \mathrm{H}, 2.56 ; \mathrm{N}$, 3.56. Found: C, 54.98; H, 2.60; N, 3.55.

2-Methoxy-5a-(trifluoromethyl)-5a $H, 11 H$-chromeno[2,3-b]chromen-11-one (2p): mp 170-171 ${ }^{\circ} \mathrm{C}$; IR (KBr) 1670, 1617, 1570, 1490, $1462 \mathrm{~cm}^{-1} ;{ }^{1} \mathrm{H}$ NMR (400 MHz, $\left.\mathrm{CDCl}_{3}\right) \delta 3.82(\mathrm{~s}, 3 \mathrm{H}), 6.90$ $(\mathrm{d}, 1 \mathrm{H}, J=2.9 \mathrm{~Hz}), 7.00(\mathrm{dd}, 1 \mathrm{H}, J=8.9,2.9 \mathrm{~Hz}), 7.08(\mathrm{~d}, 1 \mathrm{H}, J=8.9 \mathrm{~Hz}), 7.15-7.20(\mathrm{~m}, 2 \mathrm{H}), 7.60$ (ddd, $1 \mathrm{H}, J=8.4,7.3,1.7 \mathrm{~Hz}), 7.92(\mathrm{~s}, 1 \mathrm{H}), 8.01(\mathrm{dd}, 1 \mathrm{H}, J=7.8,1.7 \mathrm{~Hz}) ;{ }^{19} \mathrm{~F}$ NMR $(376 \mathrm{MHz}$, $\left.\mathrm{CDCl}_{3} / \mathrm{C}_{6} \mathrm{~F}_{6}\right) \delta 76.06$ (s). Anal. Calcd for $\mathrm{C}_{18} \mathrm{H}_{11} \mathrm{~F}_{3} \mathrm{O}_{4}$ : C, 62.08; H, 3.18. Found: $\mathrm{C}, 61.93 ; \mathrm{H}, 3.11$.

2-Methoxy-9-methyl-5a-(trifluoromethyl)-5a $H, 11 H$-chromeno[2,3-b]chromen-11-one $\quad(2 q)$ : mp 194-195 ${ }^{\circ} \mathrm{C}$; IR (KBr) 1671, 1619, 1573, 1488, 1463, $1418 \mathrm{~cm}^{-1} ;{ }^{1} \mathrm{H}$ NMR $\left(400 \mathrm{MHz}, \mathrm{CDCl}_{3}\right) \delta$ $2.36(\mathrm{~s}, 3 \mathrm{H}), 3.82(\mathrm{~s}, 3 \mathrm{H}), 6.89(\mathrm{~d}, 1 \mathrm{H}, J=2.9 \mathrm{~Hz}), 6.99(\mathrm{dd}, 1 \mathrm{H}, J=8.9,2.8 \mathrm{~Hz}), 7.04-7.09(\mathrm{~m}$, 2H), 7.40 (br.dd, $1 \mathrm{H}, J=8.5,2.0 \mathrm{~Hz}$ ), 7.80 (br.s, 1H), 7.91 (s, 1H); ${ }^{19} \mathrm{~F}$ NMR (376 MHz, $\left.\mathrm{CDCl}_{3} / \mathrm{C}_{6} \mathrm{~F}_{6}\right) \delta 76.20$ (s). Anal. Calcd for $\mathrm{C}_{19} \mathrm{H}_{13} \mathrm{~F}_{3} \mathrm{O}_{4}$ : C, 62.99; H, 3.62. Found: $\mathrm{C}, 63.06 ; \mathrm{H}, 3.63$.

5a-(1,1,2,2-Tetrafluoroethyl)-5a $H, 11 H$-chromeno[2,3-b]chromen-11-one (2s): mp 119-120 ${ }^{\circ} \mathrm{C}$; IR (KBr) 1679, 1630, 1609, 1572, 1487, $1465 \mathrm{~cm}^{-1} ;{ }^{1} \mathrm{H}$ NMR $\left(400 \mathrm{MHz}, \mathrm{CDCl}_{3}\right) \delta 6.23(\mathrm{tt}, 1 \mathrm{H}, J=$ 52.4, 6.0 Hz), 7.06-7.21 (m, 4H), 7.40-7.46 (m, 2H), $7.60(\mathrm{ddd}, 1 \mathrm{H}, J=8.5,7.2,1.7 \mathrm{~Hz}), 7.96(\mathrm{~s}$, $1 \mathrm{H}), 8.02(\mathrm{dd}, 1 \mathrm{H}, J=7.8,1.7 \mathrm{~Hz}) ;{ }^{19} \mathrm{~F}$ NMR $\left(376 \mathrm{MHz}, \mathrm{CDCl}_{3} / \mathrm{C}_{6} \mathrm{~F}_{6}\right) \delta 25.21$ (dddd, $1 \mathrm{~F}, J=304.4$, 52.6, 8.7, $5.0 \mathrm{~Hz}$ ), 26.13 (dddd, 1F, $J=304.4,52.6,8.7,5.0 \mathrm{~Hz}), 31.24(\mathrm{dm}, 1 \mathrm{~F}, J=280.4 \mathrm{~Hz}), 33.62$ (ddt, $1 \mathrm{~F}, J=280.4,8.7,5.2 \mathrm{~Hz}$ ). Anal. Calcd for $\mathrm{C}_{18} \mathrm{H}_{10} \mathrm{~F}_{4} \mathrm{O}_{3}$ : C, 61.72; H, 2.88. Found: C, 61.59; $\mathrm{H}$, 2.63 .

9-Chloro-5a-(perfluoroethyl)-5aH,11H-chromeno[2,3-b]chromen-11-one (2t): $\mathrm{mp} 150-151{ }^{\circ} \mathrm{C}$; IR (KBr) 1676, 1624, 1608, 1569, 1470, $1424 \mathrm{~cm}^{-1} ;{ }^{1} \mathrm{H}$ NMR (400 MHz, $\left.\mathrm{CDCl}_{3}\right) \delta$ 7.07-7.17 (m, 3H), 7.41-7.49 (m, 2H), $7.54(\mathrm{dd}, 1 \mathrm{H}, J=8.8,2.7 \mathrm{~Hz}), 7.98(\mathrm{~d}, 1 \mathrm{H}, J=2.7 \mathrm{~Hz}), 8.02(\mathrm{~s}, 1 \mathrm{H}) ;{ }^{19} \mathrm{~F}$ NMR (376 MHz, $\left.\mathrm{CDCl}_{3} / \mathrm{C}_{6} \mathrm{~F}_{6}\right) \delta 35.65(\mathrm{~d}, 1 \mathrm{~F}, J=288.4 \mathrm{~Hz}), 37.50(\mathrm{~d}, 1 \mathrm{~F}, J=288.4 \mathrm{~Hz}), 82.52(\mathrm{~s}$, 3F). Anal. Calcd for $\mathrm{C}_{18} \mathrm{H}_{8} \mathrm{ClF}_{5} \mathrm{O}_{3}$ : C, 53.69; H, 2.00. Found: C, 53.45; H, 1.96 . 
2-Bromo-9-chloro-5a-(perfluoroethyl)-5a $H, 11 H$-chromeno[2,3-b]chromen-11-one $\quad(2 \mathrm{u}): \mathrm{mp}$ $166-167{ }^{\circ} \mathrm{C}$; IR (KBr) 1678, 1624, 1606, 1560, 1469, $1430 \mathrm{~cm}^{-1} ;{ }^{1} \mathrm{H}$ NMR $\left(400 \mathrm{MHz}, \mathrm{CDCl}_{3}\right) \delta$ 6.99-7.03 (m, 1H), $7.09(\mathrm{~d}, 1 \mathrm{H}, J=8.8 \mathrm{~Hz}), 7.53-7.58(\mathrm{~m}, 3 \mathrm{H}), 7.93(\mathrm{~s}, 1 \mathrm{H}), 7.97(\mathrm{~d}, 1 \mathrm{H}, J=2.6$ $\mathrm{Hz}) ;{ }^{19} \mathrm{~F}$ NMR $\left(376 \mathrm{MHz}, \mathrm{CDCl}_{3} / \mathrm{C}_{6} \mathrm{~F}_{6}\right) \delta 35.88(\mathrm{~d}, 1 \mathrm{~F}, J=288.9 \mathrm{~Hz}), 37.71(\mathrm{~d}, 1 \mathrm{~F}, J=288.9 \mathrm{~Hz})$, 82.53 (s, 3F). Anal. Calcd for $\mathrm{C}_{18} \mathrm{H}_{7} \mathrm{BrClF}_{5} \mathrm{O}_{3}$ : C, 44.89; H, 1.47. Found: C, 44.70; H, 1.26.

9-Chloro-5a-(trichloromethyl)-5a H,11H-chromeno[2,3-b]chromen-11-one (2v): mp 144-145 ${ }^{\circ} \mathrm{C}$; IR (KBr) 1676, 1620, 1605, 1569, 1468, $1422 \mathrm{~cm}^{-1} ;{ }^{1} \mathrm{H}$ NMR (400 MHz, $\left.\mathrm{CDCl}_{3}\right) \delta 7.09-7.19$ (m, 3H), 7.42-7.54 (m, 3H), $7.96(\mathrm{~d}, 1 \mathrm{H}, J=2.7 \mathrm{~Hz}), 8.07(\mathrm{~s}, 1 \mathrm{H})$. Anal. Calcd for $\mathrm{C}_{17} \mathrm{H}_{8} \mathrm{Cl}_{4} \mathrm{O}_{3}: \mathrm{C}$, 50.79; H, 2.01. Found: C, 50.65; H, 2.00.

5a-(Trichloromethyl)-5a $H, 11 H$-chromeno[2,3-b]chromen-11-one (2w): mp $165-166{ }^{\circ} \mathrm{C}$; IR (KBr) 1675, 1623, 1607, 1569, $1463 \mathrm{~cm}^{-1} ;{ }^{1} \mathrm{H}$ NMR (400 MHz, $\left.\mathrm{CDCl}_{3}\right) \delta$ 7.00-7.19 (m, 4H), 7.41$7.48(\mathrm{~m}, 2 \mathrm{H}), 7.58(\mathrm{ddd}, 1 \mathrm{H}, J=8.5,7.3,1.7 \mathrm{~Hz}), 8.00(\mathrm{dd}, 1 \mathrm{H}, J=8.0,1.7 \mathrm{~Hz}), 8.04(\mathrm{~s}, 1 \mathrm{H})$. Anal. Calcd for $\mathrm{C}_{17} \mathrm{H}_{9} \mathrm{Cl}_{3} \mathrm{O}_{3}:$ C, 55.54; $\mathrm{H}, 2.47$. Found: $\mathrm{C}, 55.31 ; \mathrm{H}, 2.37$.

9-Chloro-2-methoxy-5a-(trichloromethyl)-5a $H, 11 H$-chromeno[2,3-b]chromen-11-one $\quad(2 x)$ : mp 205-206 ${ }^{\circ} \mathrm{C}$; IR (KBr) 1677, 1625, 1606, 1577, 1489, 1467, $1419 \mathrm{~cm}^{-1} ;{ }^{1} \mathrm{H}$ NMR (400 MHz, $\left.\mathrm{CDCl}_{3}\right) \delta 3.82(\mathrm{~s}, 3 \mathrm{H}), 6.91(\mathrm{~d}, 1 \mathrm{H}, J=2.9 \mathrm{~Hz}), 7.04(\mathrm{dd}, 1 \mathrm{H}, J=8.9,2.9 \mathrm{~Hz}), 7.09-7.12(\mathrm{~m}, 2 \mathrm{H})$, $7.51(\mathrm{dd}, 1 \mathrm{H}, J=8.8,2.6 \mathrm{~Hz}), 7.95(\mathrm{~d}, 1 \mathrm{H}, J=2.6 \mathrm{~Hz}), 8.02(\mathrm{~s}, 1 \mathrm{H})$. Anal. Calcd for $\mathrm{C}_{18} \mathrm{H}_{10} \mathrm{Cl}_{4} \mathrm{O}_{4}: \mathrm{C}$, 50.04; H, 2.33. Found: C, 49.91; H, 2.22.

2-Methoxy-5a-(trichloromethyl)-5aH,11H-chromeno[2,3-b]chromen-11-one (2y): mp 164-165 ${ }^{\circ} \mathrm{C}$; IR (KBr) 1676, 1624, 1578, 1490, $1462 \mathrm{~cm}^{-1}$; ${ }^{1} \mathrm{H}$ NMR (400 MHz, $\left.\mathrm{CDCl}_{3}\right) \delta 3.82(\mathrm{~s}, 3 \mathrm{H}), 6.90$ $(\mathrm{d}, 1 \mathrm{H}, J=2.9 \mathrm{~Hz}), 7.02(\mathrm{dd}, 1 \mathrm{H}, J=8.9,2.9 \mathrm{~Hz}), 7.10(\mathrm{~d}, 1 \mathrm{H}, J=8.9 \mathrm{~Hz}), 7.12-7.17(\mathrm{~m}, 2 \mathrm{H}), 7.58$ (ddd, 1H, $J=8.5,7.3,1.7 \mathrm{~Hz}), 7.99(\mathrm{dd}, 1 \mathrm{H}, J=7.8,1.7 \mathrm{~Hz}), 8.00$ (s, 1H). Found: C, 54.27; H, 2.68. Anal. Calcd for $\mathrm{C}_{18} \mathrm{H}_{11} \mathrm{Cl}_{3} \mathrm{O}_{4}$ : C, 54.37; H, 2.79.

2,2-Dimethyl-10a-(trifluoromethyl)-2,3-dihydro-4H,10a $H$-pyrano[2,3-b]chromen-4-one (3a): mp 137-138 ${ }^{\circ} \mathrm{C}$; IR (KBr) 1698, 1619, 1606, 1569, 1482, $1458 \mathrm{~cm}^{-1} ;{ }^{1} \mathrm{H}$ NMR $\left(400 \mathrm{MHz}, \mathrm{CDCl}_{3}\right) \delta$ 
$1.45(\mathrm{~s}, 3 \mathrm{H}), 1.51(\mathrm{~s}, 3 \mathrm{H}), 2.53(\mathrm{~d}, 1 \mathrm{H}, J=17.6 \mathrm{~Hz}), 2.75(\mathrm{~d}, 1 \mathrm{H}, J=17.6 \mathrm{~Hz}), 7.02-7.07(\mathrm{~m}, 2 \mathrm{H})$, $7.34(\mathrm{dd}, 1 \mathrm{H}, J=7.8,1.7 \mathrm{~Hz}), 7.38(\mathrm{ddd}, 1 \mathrm{H}, J=8.3,7.4,1.7 \mathrm{~Hz}), 7.76(\mathrm{~s}, 1 \mathrm{H}) ;{ }^{19} \mathrm{~F}$ NMR $(376 \mathrm{MHz}$, $\left.\mathrm{CDCl}_{3} / \mathrm{C}_{6} \mathrm{~F}_{6}\right) \delta 74.50$ (s). Anal. Calcd for $\mathrm{C}_{15} \mathrm{H}_{13} \mathrm{~F}_{3} \mathrm{O}_{3}: \mathrm{C}, 60.41 ; \mathrm{H}, 4.39$. Found: $\mathrm{C}, 60.59 ; \mathrm{H}, 4.28$.

\section{7-Bromo-2,2-dimethyl-10a-(trifluoromethyl)-2,3-dihydro-4 $H, 10 \mathrm{a} H$-pyrano[2,3-b]chromen-4-}

one (3b): mp 118-119 ${ }^{\circ} \mathrm{C}$; IR (KBr) 1700, 1618, 1558, $1470 \mathrm{~cm}^{-1} ;{ }^{1} \mathrm{H}$ NMR (400 MHz, $\left.\mathrm{CDCl}_{3}\right) \delta$ $1.43(\mathrm{~s}, 3 \mathrm{H}), 1.51(\mathrm{~s}, 3 \mathrm{H}), 2.55(\mathrm{~d}, 1 \mathrm{H}, J=17.7 \mathrm{~Hz}), 2.75(\mathrm{~d}, 1 \mathrm{H}, J=17.7 \mathrm{~Hz}), 6.94(\mathrm{~m}, 1 \mathrm{H})$, 7.44-7.48 (m, 2H), $7.66(\mathrm{~s}, 1 \mathrm{H}) ;{ }^{19} \mathrm{~F}$ NMR (376 MHz, $\left.\mathrm{CDCl}_{3} / \mathrm{C}_{6} \mathrm{~F}_{6}\right) \delta 74.64$ (s). Anal. Calcd for $\mathrm{C}_{15} \mathrm{H}_{12} \mathrm{BrF}_{3} \mathrm{O}_{3}$ : C, 47.77; H, 3.21. Found: C, 47.94; H, 3.19.

10a-(Difluoromethyl)-2,2-dimethyl-2,3-dihydro-4H,10a $H$-pyrano[2,3-b]chromen-4-one $\quad(3 d)$ : mp 85-86 ${ }^{\circ} \mathrm{C}$; IR (KBr) 1694, 1619, 1603, 1568, 1480, $1456 \mathrm{~cm}^{-1} ;{ }^{1} \mathrm{H}$ NMR (400 MHz, $\left.\mathrm{CDCl}_{3}\right) \delta$ $1.46(\mathrm{~s}, 3 \mathrm{H}), 1.48(\mathrm{~s}, 3 \mathrm{H}), 2.49$ (d, 1H, $J=17.5 \mathrm{~Hz}), 2.72$ (br.d, $1 \mathrm{H}, J=17.5 \mathrm{~Hz}), 5.61(\mathrm{t}, 1 \mathrm{H}, J=$ $55.4 \mathrm{~Hz}), 6.98-7.03(\mathrm{~m}, 2 \mathrm{H}), 7.30-7.37(\mathrm{~m}, 2 \mathrm{H}), 7.71(\mathrm{~s}, 1 \mathrm{H}) ;{ }^{19} \mathrm{~F}$ NMR $\left(376 \mathrm{MHz}, \mathrm{CDCl}_{3} / \mathrm{C}_{6} \mathrm{~F}_{6}\right) \delta$ $25.12(\mathrm{ddd}, 1 \mathrm{~F}, J=282.4,55.3,1.3 \mathrm{~Hz}), 27.81(\mathrm{dd}, 1 \mathrm{~F}, J=282.4,55.6 \mathrm{~Hz})$. Anal. Calcd for $\mathrm{C}_{15} \mathrm{H}_{14} \mathrm{~F}_{2} \mathrm{O}_{3}$ : C, 64.28; H, 5.03. Found: C, 64.02; H, 4.88.

9,9-Dimethyl-7a-(trifluoromethyl)-9,10-dihydro-7a $H, 11 H$-benzo[f]pyrano[2,3-b]chromen-11one (3e): $\mathrm{mp} 183-184{ }^{\circ} \mathrm{C}$; IR (KBr) $1689,1609,1559,1458 \mathrm{~cm}^{-1} ;{ }^{1} \mathrm{H}$ NMR (400 MHz, $\left.\mathrm{CDCl}_{3}\right) \delta$ $1.47(\mathrm{~s}, 3 \mathrm{H}), 1.54(\mathrm{~s}, 3 \mathrm{H}), 2.58(\mathrm{~d}, 1 \mathrm{H}, J=17.4 \mathrm{~Hz}), 2.79(\mathrm{~d}, 1 \mathrm{H}, J=17.4 \mathrm{~Hz}), 7.25(\mathrm{~d}, 1 \mathrm{H}, J=8.9$ Hz), $7.47(\mathrm{ddd}, 1 \mathrm{H}, J=8.1,7.0,1.0 \mathrm{~Hz}), 7.63(\mathrm{ddd}, 1 \mathrm{H}, J=8.3,7.0,1.3 \mathrm{~Hz}), 7.82(\mathrm{~d}, 1 \mathrm{H}, J=8.1$ $\mathrm{Hz}), 7.91(\mathrm{~d}, 1 \mathrm{H}, J=8.9 \mathrm{~Hz}), 8.12(\mathrm{~d}, 1 \mathrm{H}, J=8.3 \mathrm{~Hz}), 8.51(\mathrm{~s}, 1 \mathrm{H}) ;{ }^{19} \mathrm{~F} \mathrm{NMR}(376 \mathrm{MHz}$, $\left.\mathrm{CDCl}_{3} / \mathrm{C}_{6} \mathrm{~F}_{6}\right) \delta 74.06(\mathrm{~s})$. Anal. Calcd for $\mathrm{C}_{19} \mathrm{H}_{15} \mathrm{~F}_{3} \mathrm{O}_{3}$ : C, 65.52; H, 4.34. Found: C, 65.23; H, 4.30.

9a-(Difluoromethyl)-2,2-dimethyl-9aH-furo[2,3-b]chromen-3(2H)-one (3f): $\mathrm{mp} 76-77{ }^{\circ} \mathrm{C}$; IR $(\mathrm{KBr}) 1713,1629,1606,1565,1477,1456 \mathrm{~cm}^{-1} ;{ }^{1} \mathrm{H}$ NMR $\left(400 \mathrm{MHz}, \mathrm{CDCl}_{3}\right) \delta 1.43(\mathrm{~s}, 3 \mathrm{H}), 1.50(\mathrm{~s}$, 3H), $5.71(\mathrm{t}, 1 \mathrm{H}, J=55.0 \mathrm{~Hz}), 7.04-7.09(\mathrm{~m}, 2 \mathrm{H}), 7.37-7.42(\mathrm{~m}, 2 \mathrm{H}), 7.56(\mathrm{~s}, 1 \mathrm{H}) ;{ }^{19} \mathrm{~F}$ NMR $(376$ $\left.\mathrm{MHz}, \mathrm{CDCl}_{3} / \mathrm{C}_{6} \mathrm{~F}_{6}\right) \delta 24.86(\mathrm{dd}, 1 \mathrm{~F}, J=287.6,54.9 \mathrm{~Hz}), 28.93(\mathrm{dd}, 1 \mathrm{~F}, J=287.6,55.1 \mathrm{~Hz})$. Anal. Calcd for $\mathrm{C}_{14} \mathrm{H}_{12} \mathrm{~F}_{2} \mathrm{O}_{3}$ : C, 63.16; H, 4.54. Found: C, 63.28; H, 4.39. 

140-141 ${ }^{\circ} \mathrm{C}$; IR (KBr) 1726, 1637, 1605, 1566, $1452 \mathrm{~cm}^{-1} ;{ }^{1} \mathrm{H}$ NMR (400 MHz, $\left.\mathrm{CDCl}_{3}\right) \delta 1.3-2.1$ $(\mathrm{m}, 10 \mathrm{H}), 7.06(\mathrm{td}, 1 \mathrm{H}, J=7.5,1.0 \mathrm{~Hz}), 7.15(\mathrm{~d}, 1 \mathrm{H}, J=8.2 \mathrm{~Hz}), 7.38-7.45(\mathrm{~m}, 2 \mathrm{H}), 7.62(\mathrm{~s}, 1 \mathrm{H})$; ${ }^{13} \mathrm{C} \mathrm{NMR}\left(100 \mathrm{MHz}, \mathrm{CDCl}_{3}\right) \delta 21.1,21.4,24.7,31.0,36.3,87.8,103.3,104.2,116.1,119.3,121.3$, 122.8, 130.4, 133.8, 134.4, 154.7, 199.5. Anal. Calcd for $\mathrm{C}_{17} \mathrm{H}_{15} \mathrm{Cl}_{3} \mathrm{O}_{3}$ : C, 54.64; $\mathrm{H}, 4.05$. Found: $\mathrm{C}$, $54.65 ; \mathrm{H}, 3.76$.

trans-5a,6a-Bis(difluoromethyl)-5a $H, 6 a H, 13 H$-chromeno[3',2' :5,6]pyrano[2,3-b]chromen-13one (trans-6b): yield 73\%; mp 212-213 ${ }^{\circ} \mathrm{C}$; IR (Nujol) 1679, 1628, 1605, $1569 \mathrm{~cm}^{-1}$; ${ }^{1} \mathrm{H}$ NMR (400

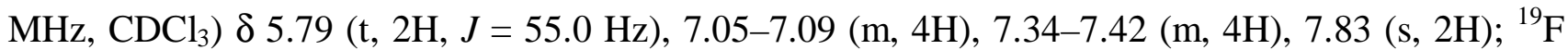
NMR (376 MHz, $\left.\mathrm{CDCl}_{3} / \mathrm{C}_{6} \mathrm{~F}_{6}\right) \delta 27.13(\mathrm{dd}, 2 \mathrm{~F}, J=286.1,55.0 \mathrm{~Hz}), 28.66(\mathrm{dd}, 2 \mathrm{~F}, J=286.1,55.0$ $\mathrm{Hz}) ;{ }^{13} \mathrm{C} \mathrm{NMR}\left(100 \mathrm{MHz}, \mathrm{CDCl}_{3}\right) \delta 98.7(\mathrm{t}, J=28.0 \mathrm{~Hz}), 113.1(\mathrm{t}, J=254.0 \mathrm{~Hz}), 115.9,118.1$, $119.4(\mathrm{~d}, J=0.7 \mathrm{~Hz}), 123.1,130.5,133.9,136.1,152.5(\mathrm{~d}, J=1.0 \mathrm{~Hz}), 180.0$. Anal. Calcd for $\mathrm{C}_{21} \mathrm{H}_{12} \mathrm{~F}_{4} \mathrm{O}_{4}: \mathrm{C}, 62.38 ; \mathrm{H}, 2.99 ; \mathrm{F}, 18.80$. Found: C, 62.41; H, 2.93; F, 18.77.

cis-5a,6a-Bis(difluoromethyl)-5aH,6a $H, 13 H$-chromeno[3',2':5,6]pyrano[2,3-b]chromen-13one (cis-6b): yield 53\%; mp 166-167 ${ }^{\circ} \mathrm{C}$; IR (Nujol) 1679, 1618, 1605, $1567 \mathrm{~cm}^{-1} ;{ }^{1} \mathrm{H}$ NMR (400

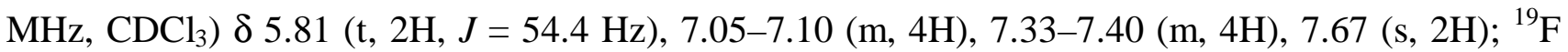
NMR $\left(376 \mathrm{MHz}, \mathrm{CDCl}_{3} / \mathrm{C}_{6} \mathrm{~F}_{6}\right) \delta 26.98(\mathrm{ddm}, 2 \mathrm{~F}, J=285.0,54.2 \mathrm{~Hz}), 28.20(\mathrm{ddm}, 2 \mathrm{~F}, J=285.0$, 54.2 Hz). Anal. Calcd for $\mathrm{C}_{21} \mathrm{H}_{12} \mathrm{~F}_{4} \mathrm{O}_{4}$ : C, 62.38; H, 2.99; F, 18.80. Found: C, 62.60; H, 2.91; F, 18.87.

\section{trans-5a,6a-Bis(1,1,2,2-tetrafluoroethyl)-5a $H, 6 a H, 13 H$-chromeno[3',2':5,6]pyrano[2,3-b]-}

chromen-13-one (trans-6c): yield 68\%; mp 186-187 ${ }^{\circ} \mathrm{C}$; IR (Nujol) 1674, 1624, 1607, $1569 \mathrm{~cm}^{-1}$; ${ }^{1} \mathrm{H}$ NMR (400 MHz, $\left.\mathrm{CDCl}_{3}\right) \delta 6.28(\mathrm{tdd}, 2 \mathrm{H}, J=52.7,9.8,2.5 \mathrm{~Hz}), 7.04-7.12(\mathrm{~m}, 4 \mathrm{H}), 7.38-7.44$ $(\mathrm{m}, 4 \mathrm{H}), 7.91(\mathrm{~s}, 2 \mathrm{H}) ;{ }^{19} \mathrm{~F}$ NMR $\left(376 \mathrm{MHz}, \mathrm{CDCl}_{3} / \mathrm{C}_{6} \mathrm{~F}_{6}\right) \delta 23.28$ (dddd, 2F, $J=305.0,52.6,10.5$, $8.0 \mathrm{~Hz}), 27.77(\mathrm{ddd}, 2 \mathrm{~F}, J=305.0,52.4,11.3 \mathrm{~Hz}), 28.40(\mathrm{dm}, 2 \mathrm{~F}, J=274.8 \mathrm{~Hz}), 33.10(\mathrm{ddd}, 2 \mathrm{~F}, J=$ 
274.8, 10.6, 2.5 Hz). Anal. Calcd for $\mathrm{C}_{23} \mathrm{H}_{12} \mathrm{~F}_{8} \mathrm{O}_{4}$ : C, 54.77; H, 2.40; F, 30.14. Found: C, 54.93; H, $2.56 ; \mathrm{F}, 30.01$.

trans-2,10-Dibromo-5a,6a-bis(trifluoromethyl)-5a $H, 6 a H, 13 H$-chromeno[3',2' :5,6]pyrano[2,3b]chromen-13-one (trans-6d): yield 58\%; mp >240 ${ }^{\circ} \mathrm{C}$ (subl.); IR (Nujol) 1678, 1621, 1599, 1559 $\mathrm{cm}^{-1} ;{ }^{1} \mathrm{H}$ NMR $\left(400 \mathrm{MHz}, \mathrm{CDCl}_{3}\right) \delta$ 7.03-7.05 (m, 2H), 7.52-7.56 (m, 4H), $7.83(\mathrm{~s}, 2 \mathrm{H}) ;{ }^{19} \mathrm{~F}$ NMR (376 MHz, $\left.\mathrm{CDCl}_{3} / \mathrm{C}_{6} \mathrm{~F}_{6}\right) \delta 75.78$ (s). Anal. Calcd for $\mathrm{C}_{21} \mathrm{H}_{8} \mathrm{Br}_{2} \mathrm{~F}_{6} \mathrm{O}_{4}$ : C, 42.17; H, 1.35; F, 19.06 . Found: C, 42.21; H, 1.29; F, 19.14.

cis-2,10-Dibromo-5a,6a-bis(trifluoromethyl)-5a $H, 6 a H, 13 H$-chromeno[3',2':5,6]pyrano[2,3b]chromen-13-one (cis-6d): yield 30\%; mp 230-232 ${ }^{\circ} \mathrm{C}$ (subl.); IR (Nujol) 1679, 1615, 1602, 1555 $\mathrm{cm}^{-1} ;{ }^{1} \mathrm{H}$ NMR $\left(400 \mathrm{MHz}, \mathrm{CDCl}_{3}\right) \delta$ 7.02-7.04 (m, 2H), 7.49-7.52 (m, 4H), 7.56 (s, 2H); ${ }^{19} \mathrm{~F}$ NMR (376 MHz, $\left.\mathrm{CDCl}_{3} / \mathrm{C}_{6} \mathrm{~F}_{6}\right) \delta 76.48$ (s). Anal. Calcd for $\mathrm{C}_{21} \mathrm{H}_{8} \mathrm{Br}_{2} \mathrm{~F}_{6} \mathrm{O}_{4}$ : C, 42.17; H, 1.35; F, 19.06 . Found: C, 42.28; H, 1.14; F, 18.97.

trans-2,10-Dibromo-5a,6a-bis(difluoromethyl)-5a $H, 6 a H, 13 H$-chromeno[3',2' :5,6]pyrano[2,3b]chromen-13-one (trans-6e): yield 82\%; mp >240 ${ }^{\circ} \mathrm{C}$ (subl.); IR (Nujol) 1677, 1618, 1605, 1555 $\mathrm{cm}^{-1} ;{ }^{1} \mathrm{H}$ NMR $\left(400 \mathrm{MHz}, \mathrm{CDCl}_{3}\right) \delta 5.77(\mathrm{t}, 2 \mathrm{H}, J=54.9 \mathrm{~Hz}), 6.94-6.96(\mathrm{~m}, 2 \mathrm{H}), 7.48-7.50(\mathrm{~m}$, 4H), $7.74(\mathrm{~s}, 2 \mathrm{H}) ;{ }^{19} \mathrm{~F}$ NMR $\left(376 \mathrm{MHz}, \mathrm{CDCl}_{3} / \mathrm{C}_{6} \mathrm{~F}_{6}\right) \delta 27.22(\mathrm{dd}, 2 \mathrm{~F}, J=287.6,54.9 \mathrm{~Hz}), 29.02(\mathrm{dd}$, 2F, $J=287.6,54.9 \mathrm{~Hz}$ ). Anal. Calcd for $\mathrm{C}_{21} \mathrm{H}_{10} \mathrm{Br}_{2} \mathrm{~F}_{4} \mathrm{O}_{4}: \mathrm{C}, 44.87 ; \mathrm{H}, 1.79 ; \mathrm{F}, 13.52$. Found: C, 44.96; H, 1.63; F, 13.58 .

trans-5a-(Difluoromethyl)-6a-(trifluoromethyl)-5a $H, 6 a H, 13 H$-chromeno[3',2' :5,6]pyrano[2,3b]chromen-13-one (trans-6f): yield 70\%; mp 198-199 ${ }^{\circ} \mathrm{C}$; IR (Nujol) 1690, 1634, 1606, $1570 \mathrm{~cm}^{-1}$; ${ }^{1} \mathrm{H}$ NMR $\left(400 \mathrm{MHz}, \mathrm{CDCl}_{3}\right) \delta 5.81(\mathrm{t}, 1 \mathrm{H}, J=55.0 \mathrm{~Hz}), 7.06-7.12(\mathrm{~m}, 4 \mathrm{H}), 7.35-7.45(\mathrm{~m}, 4 \mathrm{H}), 7.86$ $(\mathrm{s}, 2 \mathrm{H}) ;{ }^{19} \mathrm{~F}$ NMR $\left(376 \mathrm{MHz}, \mathrm{CDCl}_{3} / \mathrm{C}_{6} \mathrm{~F}_{6}\right) \delta 27.16(\mathrm{dd}, 1 \mathrm{~F}, J=286.7,55.0 \mathrm{~Hz}), 28.95(\mathrm{dd}, 1 \mathrm{~F}, J=$ 286.7, 55.0 Hz), 75.62 (s, 3F). Found: C, 59.61; H, 2.66; F, 22.36. Anal. Calcd for $\mathrm{C}_{21} \mathrm{H}_{11} \mathrm{~F}_{5} \mathrm{O}_{4}$ : C, $59.73 ; \mathrm{H}, 2.63 ; \mathrm{F}, 22.49$. 


\section{2,10a-Bis(difluoromethyl)-2-hydroxy-2,3-dihydro-4H,10a $H$-pyrano[2,3-b]chromen-4-one (8).}

A solution of salicylaldehyde $(1.0 \mathrm{mmol})$ and piperidine $(2.0 \mathrm{mmol})$ in anhydrous benzene $(10 \mathrm{~mL})$ in the presence of a catalytic amount of $p$-toluene sulfonic acid (5 mol \%) was refluxed for $2 \mathrm{~h}$. The reaction mixture was concentrated and, after addition of $p$-TsOH $(1.0 \mathrm{mmol})$ and 2,6bis(difluoromethyl)-4H-pyran-4-one $(2.0 \mathrm{mmol})$, was heated under reflux for $4 \mathrm{~h}$. Then the resulting mixture was poured into $5 \%$ solution $\mathrm{HCl}(15 \mathrm{~mL})$ and stirred for $15 \mathrm{~min}$. After removal of the solvent, the crystalline material was isolated by filtration, dried, and recrystallized twice from $\mathrm{CCl}_{4}$ to give the title compound 8 as light-yellow powder. Yield $40 \%$; mp $146-148{ }^{\circ} \mathrm{C}$; IR (Nujol) 3276 , 1691, 1622, 1607, $1568 \mathrm{~cm}^{-1} ;{ }^{1} \mathrm{H}$ NMR (400 MHz, $\left.\mathrm{CDCl}_{3}\right) \delta 2.81(\mathrm{dd}, 1 \mathrm{H}, J=18.0 \mathrm{~Hz}), 2.83(\mathrm{dd}$, $1 \mathrm{H}, J=18.0 \mathrm{~Hz}), 3.58(\mathrm{t}, 1 \mathrm{H}, J=1.6 \mathrm{~Hz}), 5.69(\mathrm{t}, 1 \mathrm{H}, J=55.2 \mathrm{~Hz}), 5.76(\mathrm{t}, 1 \mathrm{H}, J=55.0 \mathrm{~Hz}), 6.98-$ $7.05(\mathrm{~m}, 2 \mathrm{H}), 7.32-7.36(\mathrm{~m}, 2 \mathrm{H}), 7.73(\mathrm{~s}, 1 \mathrm{H}) ;{ }^{19} \mathrm{~F} \mathrm{NMR}\left(376 \mathrm{MHz}, \mathrm{CDCl}_{3} / \mathrm{C}_{6} \mathrm{~F}_{6}\right) \delta 25.06(\mathrm{br} . \mathrm{dd}, 1 \mathrm{~F}$, $J=286.2,55.2 \mathrm{~Hz}), 27.41(\mathrm{dd}, 1 \mathrm{~F}, J=286.2,55.2 \mathrm{~Hz}), 25.45($ br.dd, $1 \mathrm{~F}, J=288.0, J=55.0 \mathrm{~Hz})$, $28.06\left(\mathrm{dd}, 1 \mathrm{~F}, J=288.0, J=55.0 \mathrm{~Hz}\right.$ ). Anal. Calcd for $\mathrm{C}_{14} \mathrm{H}_{10} \mathrm{~F}_{4} \mathrm{O}_{4}: \mathrm{C}, 52.84 ; \mathrm{H}, 3.17 ; \mathrm{F}, 23.88$. Found: C, 52.94; H, 3.23; F, 23.84.

Conversion of 8 to the monoadduct $7 \mathbf{b}$. A solution of 8 (95 $\mathrm{mg}, 0.3 \mathrm{mmol})$ in dry benzene (15 $\mathrm{mL})$ in the presence of molecular sieves $(3 \AA, 1 \mathrm{~g})$ and $p$-TsOH $(10 \mathrm{mg}, 0.06 \mathrm{mmol})$ was refluxed for $3 \mathrm{~h}$. The reaction mixture was evaporated under reduced pressure, and the resulting precipitate was purified by chromatography (silica gel, $\mathrm{CH}_{2} \mathrm{Cl}_{2}$ ). After removal of the solvent, the crystalline material was collected by filtration to give product $\mathbf{7 b}$ as light-yellow crystals, yield $58 \mathrm{mg}(64 \%)$, mp $131-132{ }^{\circ} \mathrm{C}$. 
Copy of ${ }^{1} \mathrm{H}$ NMR spectrum of $\mathbf{2 c}$

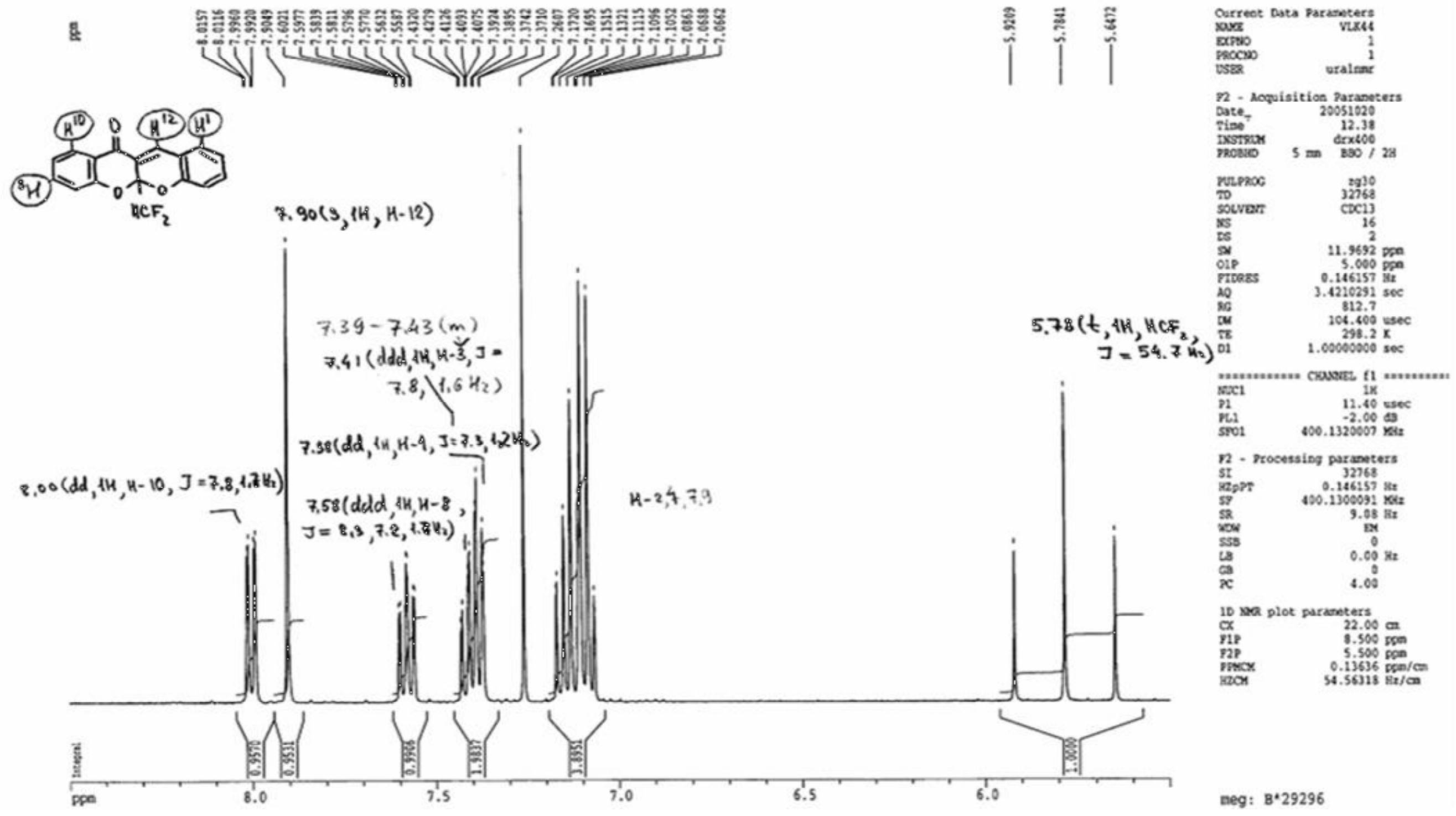

PDF created with FinePrint pdfFactory Pro trial version www.pdffactory.com 


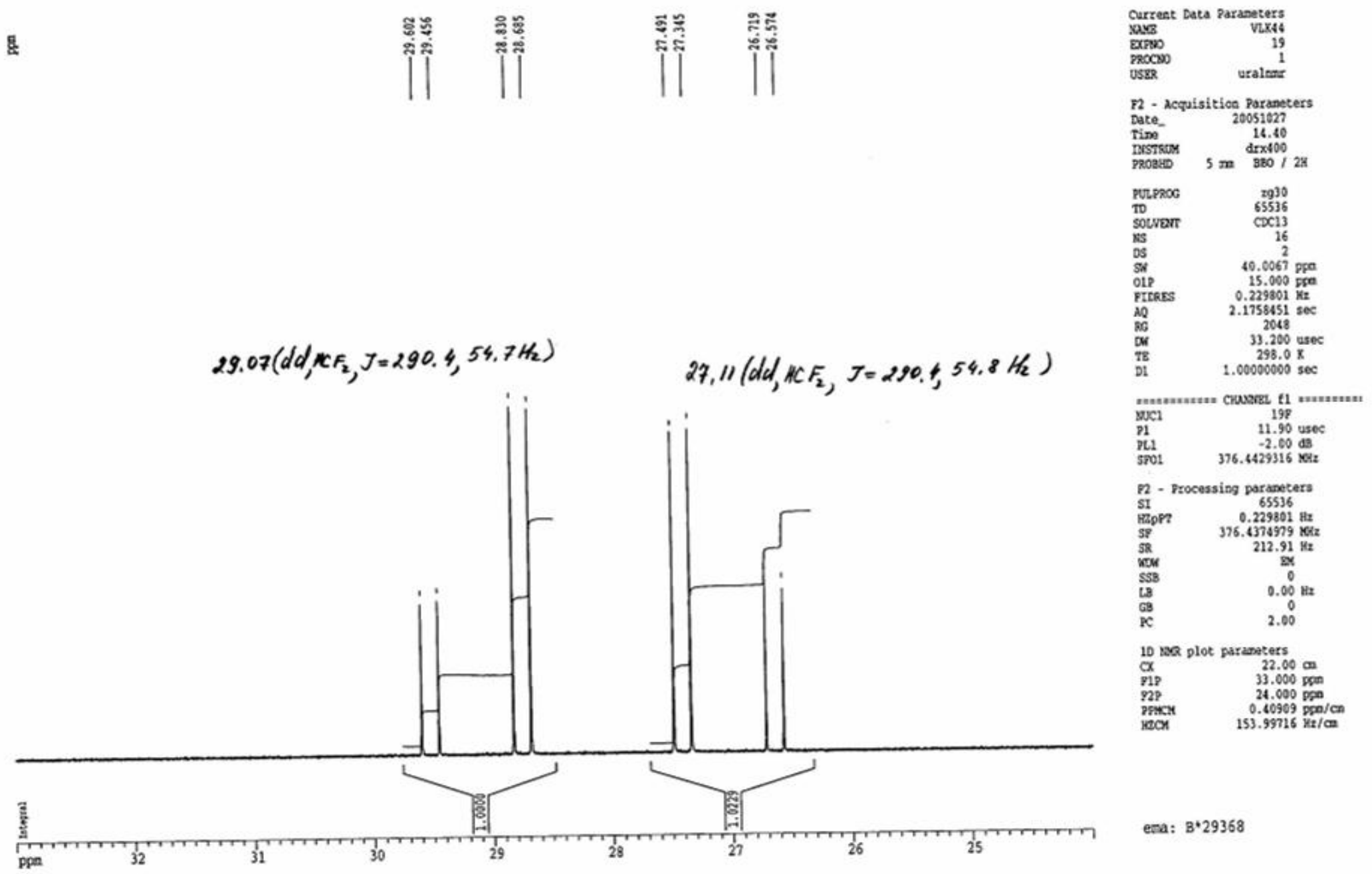


Copy of ${ }^{1} \mathrm{H}$ NMR spectrum of $\mathbf{2 e}$

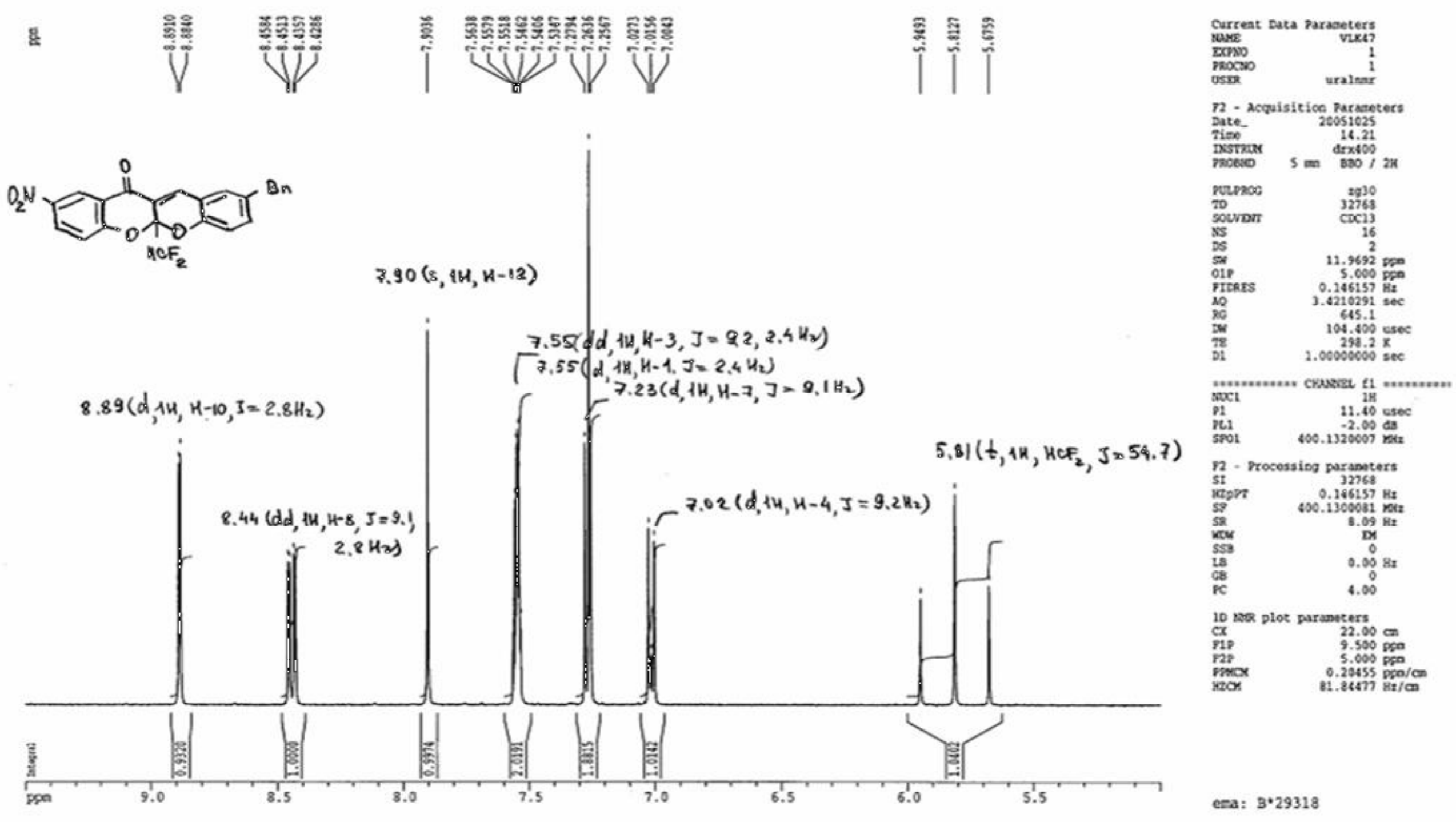


Copy of ${ }^{19}$ F NMR spectrum of $2 e$
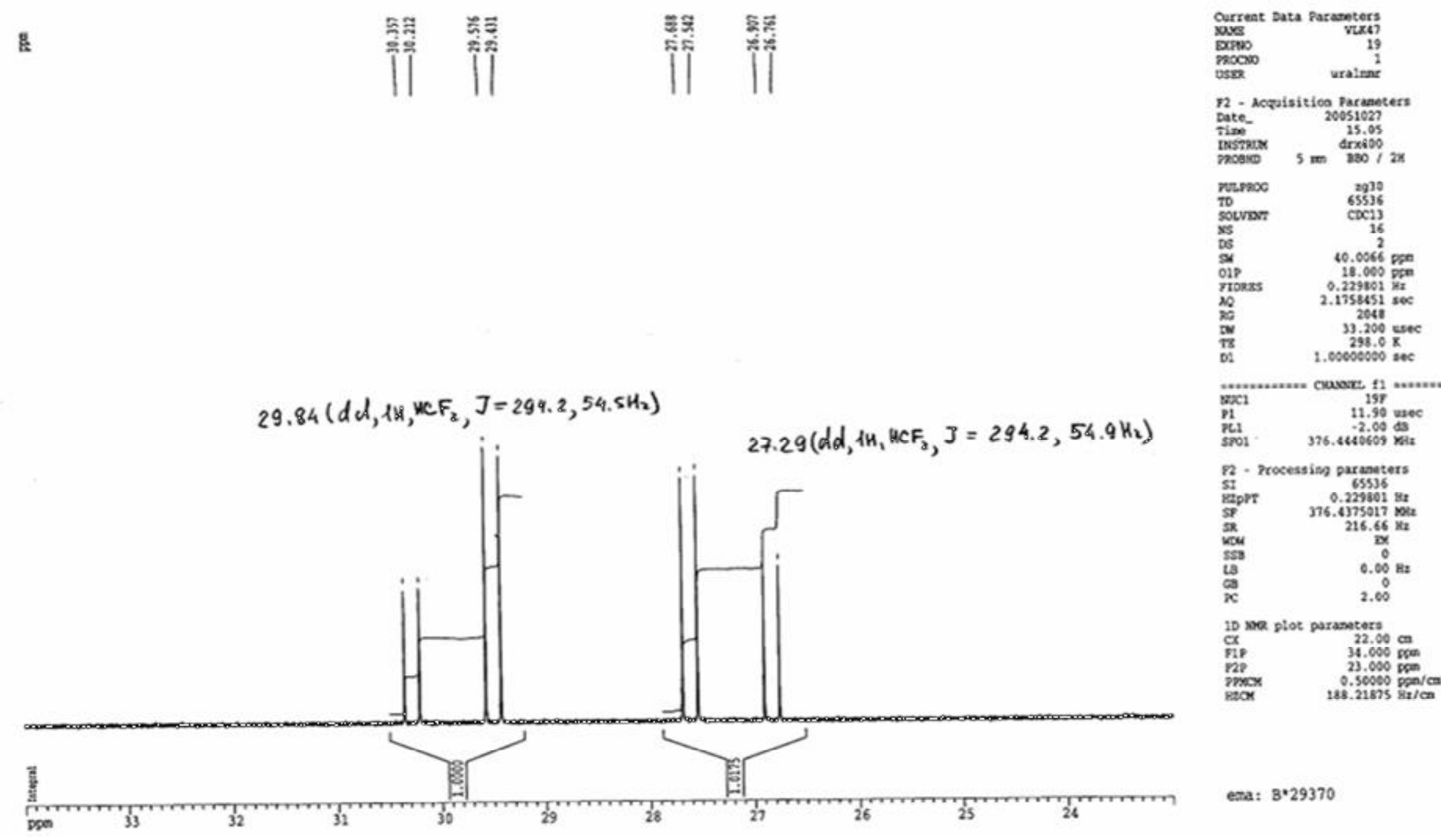

n2 - recessisn paranaters

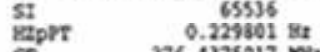

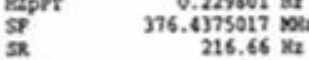

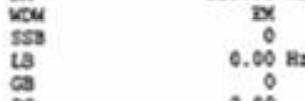

x 2.00

ID wo plot paraseters on

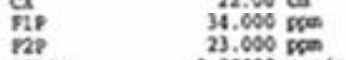

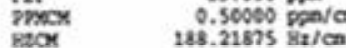

PDF created with FinePrint pdfFactory Pro trial version www.pdffactory.com 
Copy of ${ }^{1} \mathrm{H}$ NMR spectrum of $\mathbf{2 h}$

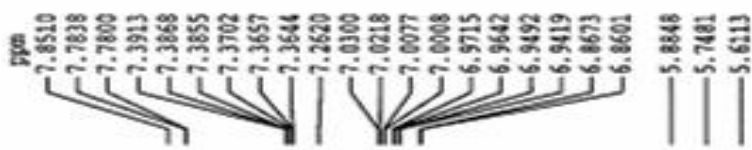
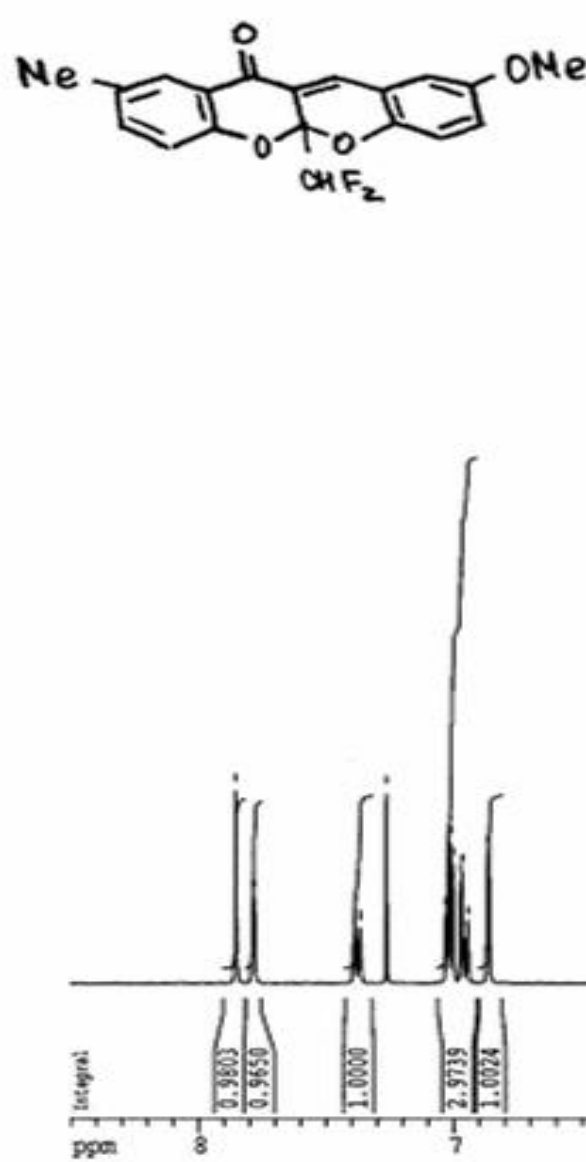

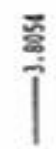

$\stackrel{\S}{\tilde{i}}$

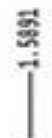

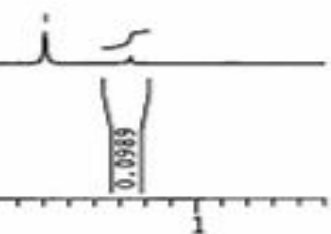

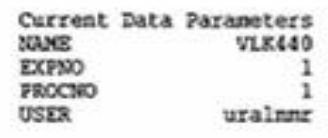

n2 - acquisiticico paraneters

Date- Acquisitico Paraneters

$\begin{array}{lr}\text { Timo } & 15.03 \\ \text { ISSTRus } & \text { drx400 }\end{array}$

$$
\mathrm{BaO} / 2 \mathrm{H}
$$

FUC.PROC 2930
32768

sotver

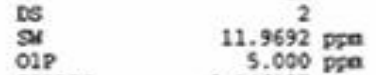

P10 $\quad \begin{aligned} & 5.000 \\ & \text { proass }\end{aligned}$

MQ $\quad 3.4210291 \mathrm{sec}$

Do $\quad 104.400$ usec

$298.0 \mathrm{~K}$

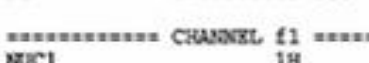

$\begin{array}{ll}\text { muc1 } & \text { in } \\ \text { P1 } & 11.40 \text { usec }\end{array}$

$\begin{array}{ll}\text { PL.1 } & \text { 2.2.00 dis } \\ \text { SFO1 } & 400.1320007 \text { vols }\end{array}$

72 - processing paradoters

SI 32768

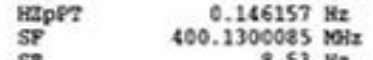

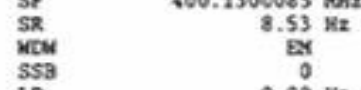

$S S B$
48
$G 8$

$\mathrm{TC}$

10 ine plot

$\mathrm{Cx}$
$\mathrm{Y1p}$
$\mathrm{y} 2 \mathrm{P}$

$22.00 \mathrm{~cm}$
$\mathrm{Fip}$

Prect $\quad 0.36364 \mathrm{pram} / \mathrm{cm}$

HAOS $145.50182 \mathrm{mz} / \mathrm{mon}$

ema: $3 * 29749$ 
Copy of ${ }^{1} \mathrm{H}$ NMR spectrum of $\mathbf{2 i}$
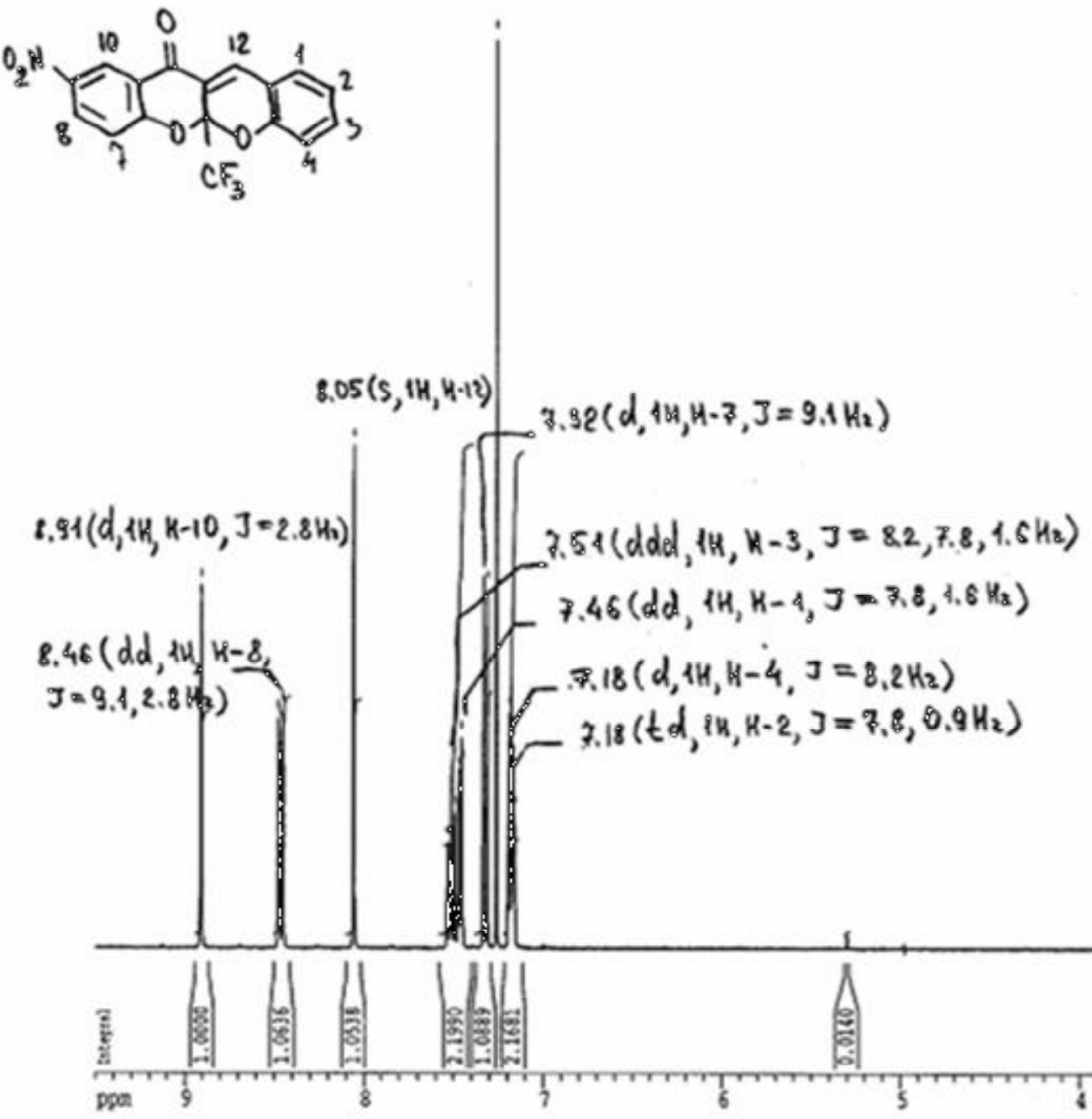

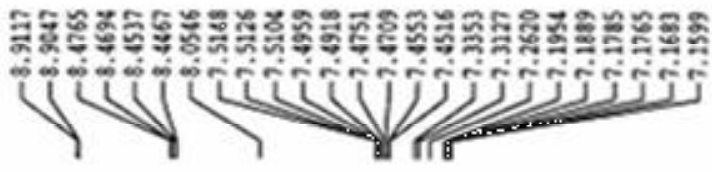

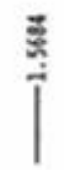

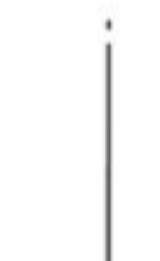$$
\text { . }
$$

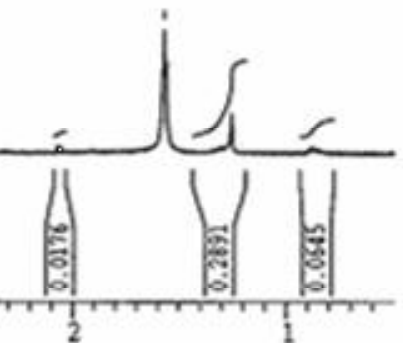

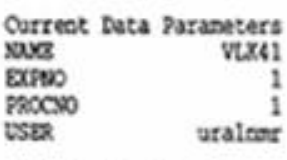

22 - Acquisition paraseters

Date20051018

mistrom nocevo $5 \mathrm{ma} 360 / 28$

Frrace $\quad 32769$

$\begin{array}{ll}\text { To } & 32768 \\ \text { souvan } & \text { caC13 }\end{array}$

is

DS
Otp
otp

olp $\quad 11.9692 \mathrm{pm}$

itwers $0.146157 \mathrm{kz}$ 3.4210291506 $812 . ?$

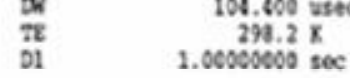

$\begin{array}{ll}\pi & 29.2 \mathrm{~K} \\ 01 & 1.00000000 \mathrm{sec}\end{array}$

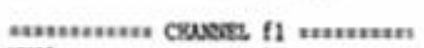

$n 1211.40$ usec

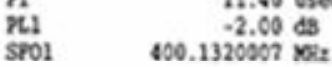

$n$ - Frocossing paradeters

SI 32768

Sy $0.166157 \mathrm{gt}$

SR $\quad 8.67 \%$

SSB

$\mathrm{LSB}$

ca

8.67 Xg

$0.00 \mathrm{ks}$

4.00

is an plot paraneters

a $\quad 22.00 \mathrm{~cm}$

290.960 ger

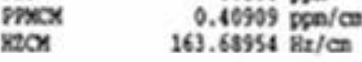

163.65954 $\mathrm{gz} / \mathrm{Cm}$ 

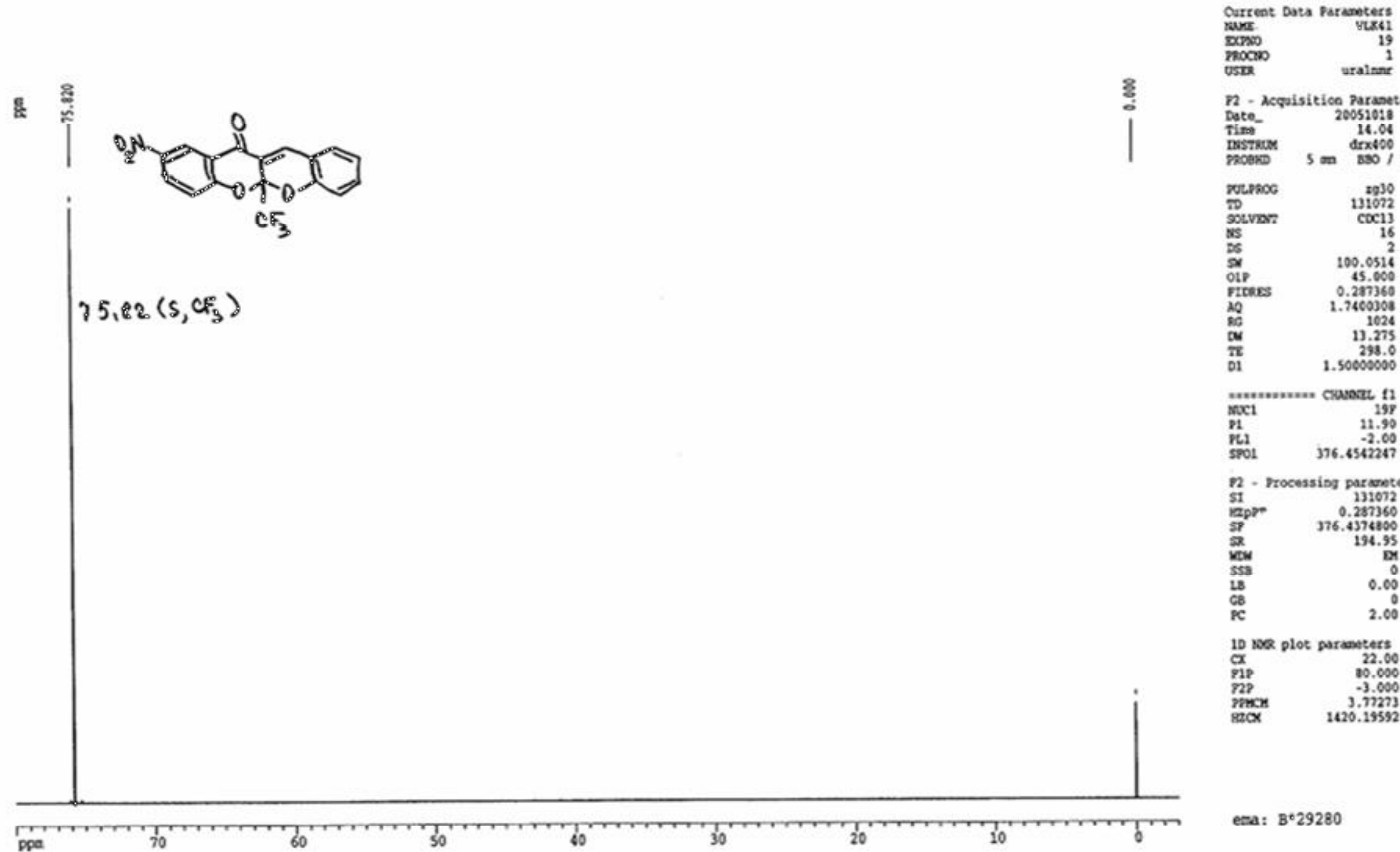
Copy of ${ }^{1} \mathrm{H}$ NMR spectrum of $\mathbf{2} \mathbf{j}$

\&
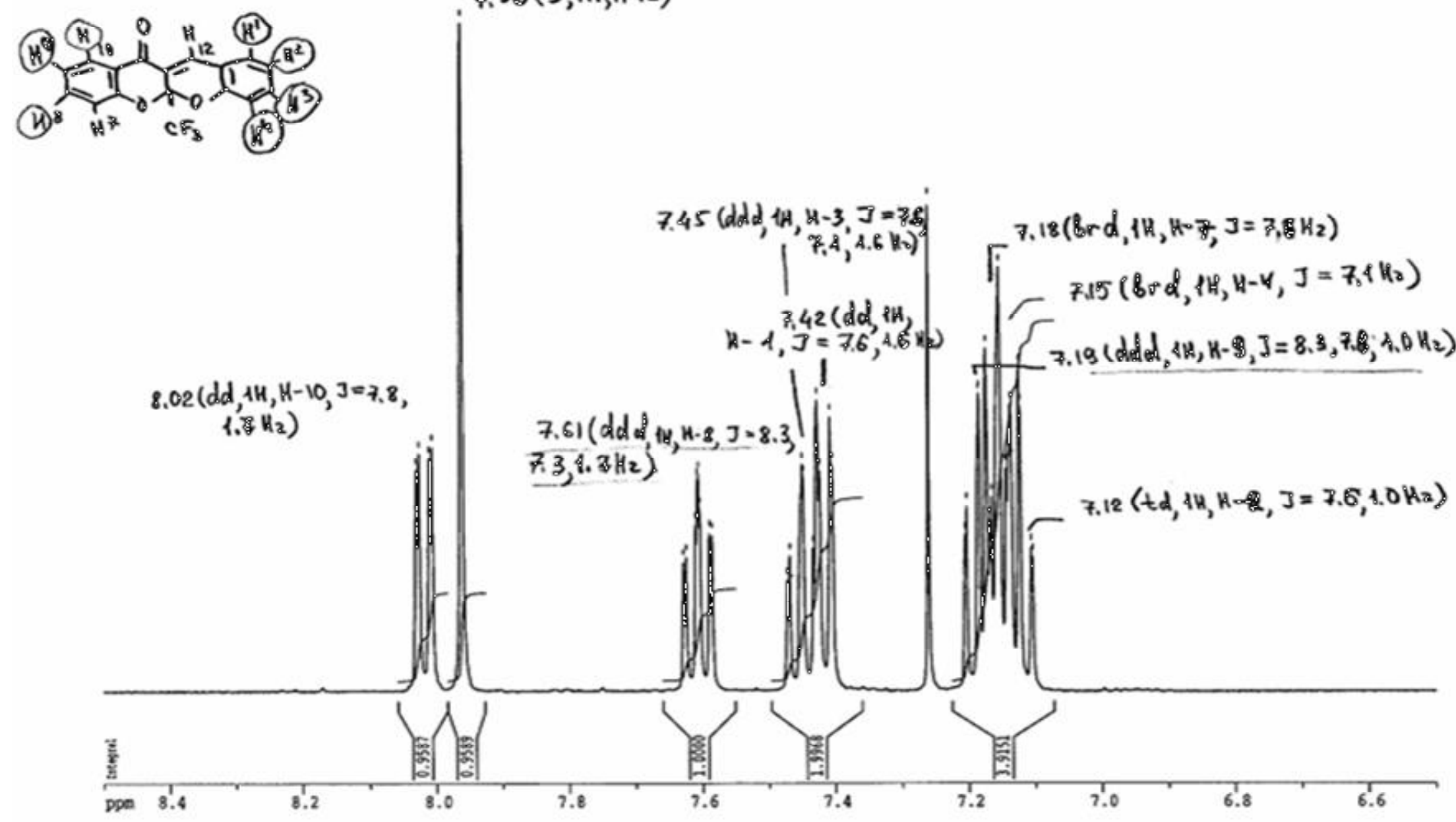

Curreat Data Paraseters

xure

pocan

$\frac{1}{1}$

$n$ - Mcquisitico paraneters

Date_ 20051020

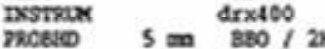

nrence

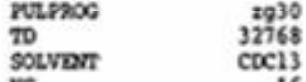

$\begin{array}{lr}\text { is } & 16 \\ \text { ts } & 2 \\ \text { sid } & 11.9599^{2}\end{array}$

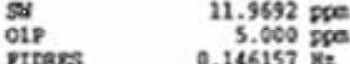

$\begin{array}{ll}0.146157 & \mathrm{Ka} \\ { }_{10} & 3.4210291 \mathrm{sec}\end{array}$

Di $\quad 1074.700$ usec

I8 $\quad 1.00000000 \mathrm{sec}$

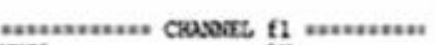

SWC1 13

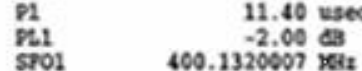

n- Frocessiog paraneters

S1 32768

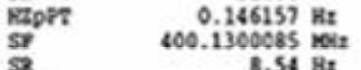

$\underset{x}{\mathrm{ce}}$

croters paraseters

$8.300 \mathrm{con}$

mor $0.09091 \mathrm{ppo} / \mathrm{co}$

ind

$36.37545 \mathrm{kz} / \mathrm{cm}$ 
量

$$
25,96\left(5, C F_{3}\right)
$$

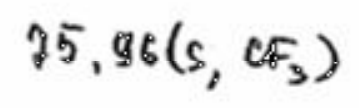

$n 2$ - Mequisition parameters

Tine- 14.29

Issiata droxion

ACBco $5 \mathrm{~min} 200 / 2 \mathrm{H}$

\section{FuL2006}

To
solven
xs

ss

is
of
oip

ntak

20

Do

DE

131072

$\mathrm{COCH}_{8}$

$89.7625 \mathrm{pme}$

0.00 .000 ppe

$0.257750 \mathrm{kz}$

$1.9399157 \mathrm{sec}$

.

11.800 usec
$298.0 \mathrm{~K}$

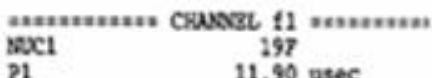

$\begin{array}{lr}71 & 11.90 \text { usec } \\ \pi 1 & -2.00 \mathrm{da}\end{array}$

$376.4523425 \mathrm{mot}$

n - Processibg parameters

SI 131072

kaph $\quad 0.257750 \mathrm{gz}$

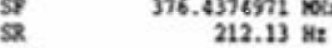

$\underset{\mathrm{NSB}}{\mathrm{NSO}} \quad$ DA

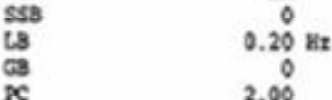

ploc paranecers

Q $22.00 \mathrm{Co}$
912
$80.000 \mathrm{pge}$

nip

$\operatorname{sincis}$

.7.77m3 $\mathrm{pga} / \mathrm{cm}$

act $1420.19604 \mathrm{kz} / \mathrm{Co}$

DF created with FinePrint pdfFactory Pro trial version www.pdffactory.com 
Copy of ${ }^{1} \mathrm{H}$ NMR spectrum of $\mathbf{2 0}$

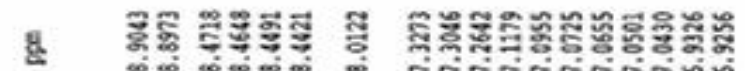
îj
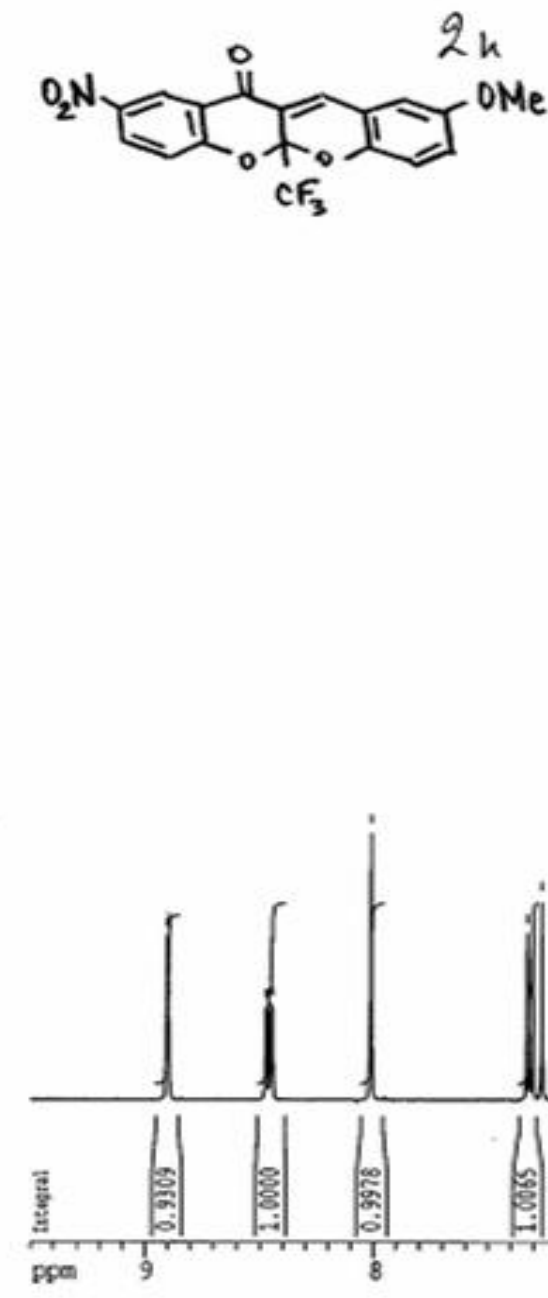
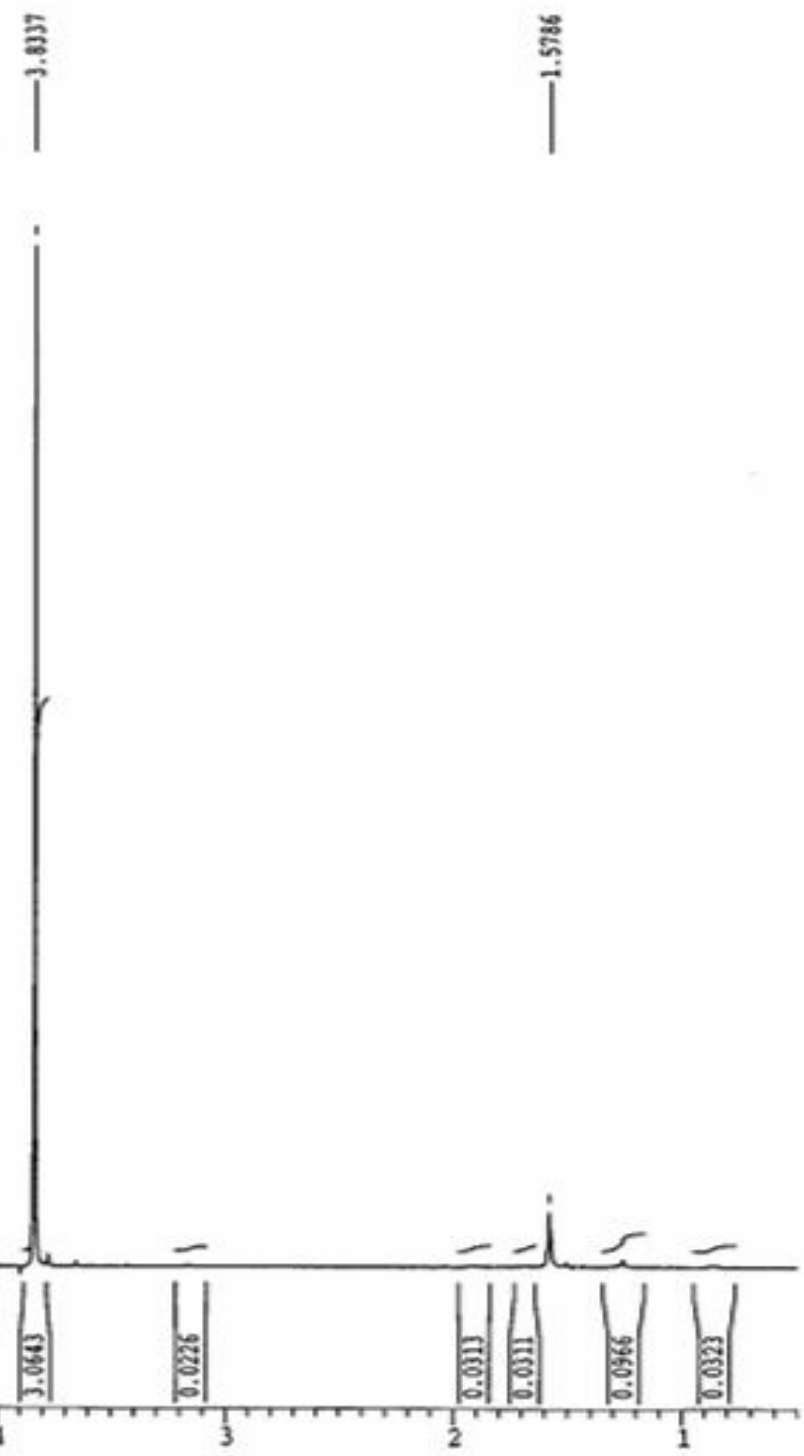

Curreat Data Parasoters

$\underset{3 x e s}{20 u s}$

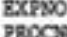

URER

$\frac{1}{1}$

72 - Acquisition Paraseters

Date_ 20051207

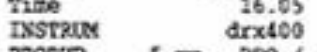

PRo6eto $5 \mathrm{~mm} 300 / 2 \mathrm{H}$

$\begin{array}{lr}\text { PULPROG } & 2930 \\ \text { TD } & 32768\end{array}$

$\begin{array}{lr}\text { sotvan }_{\text {us }} & \mathrm{COCl}_{13} \\ \text { os } & 16\end{array}$

Ot 11.9692 Dgen

$\begin{array}{ll}\text { O1P } & 5.000 \mathrm{pen} \\ \text { 7TDQR } & 0.145157 \mathrm{kz}\end{array}$

$\begin{array}{ll}\text { VTDRES } & 0.146157 \mathrm{~Hz} \\ \text { AO } & 3.4210291 \mathrm{sec}\end{array}$

A0 $\quad 3.4210291 \mathrm{sec}$

Th 104.400 use:

$\mathrm{TE}$
$\mathrm{D1}$$\quad 2.00000000 \mathrm{S0C}$

mununuse crusect $t 1$.

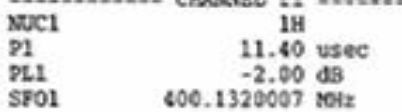

72 - Processiog paraneter:

SI 32768 .

SF $\quad 400.1300077 \mathrm{~Hz}$

SR $\quad 7.69 \mathrm{~Hz}$

SSB

다

$0.00 \mathrm{~Hz}$

id tor plot pardaeters

cx $22.00 \mathrm{cos}$

F1P
F2P

$0.500 \mathrm{prea}$

$\begin{array}{ll}\text { PPACX } & 0.40909 \mathrm{DSO} / \mathrm{Cn} \\ \text { HZCX } & 163.68954 \mathrm{~Hz} / \mathrm{Cn}\end{array}$ 
Copy of ${ }^{1} \mathrm{H}$ NMR spectrum of $\mathbf{2 r}$

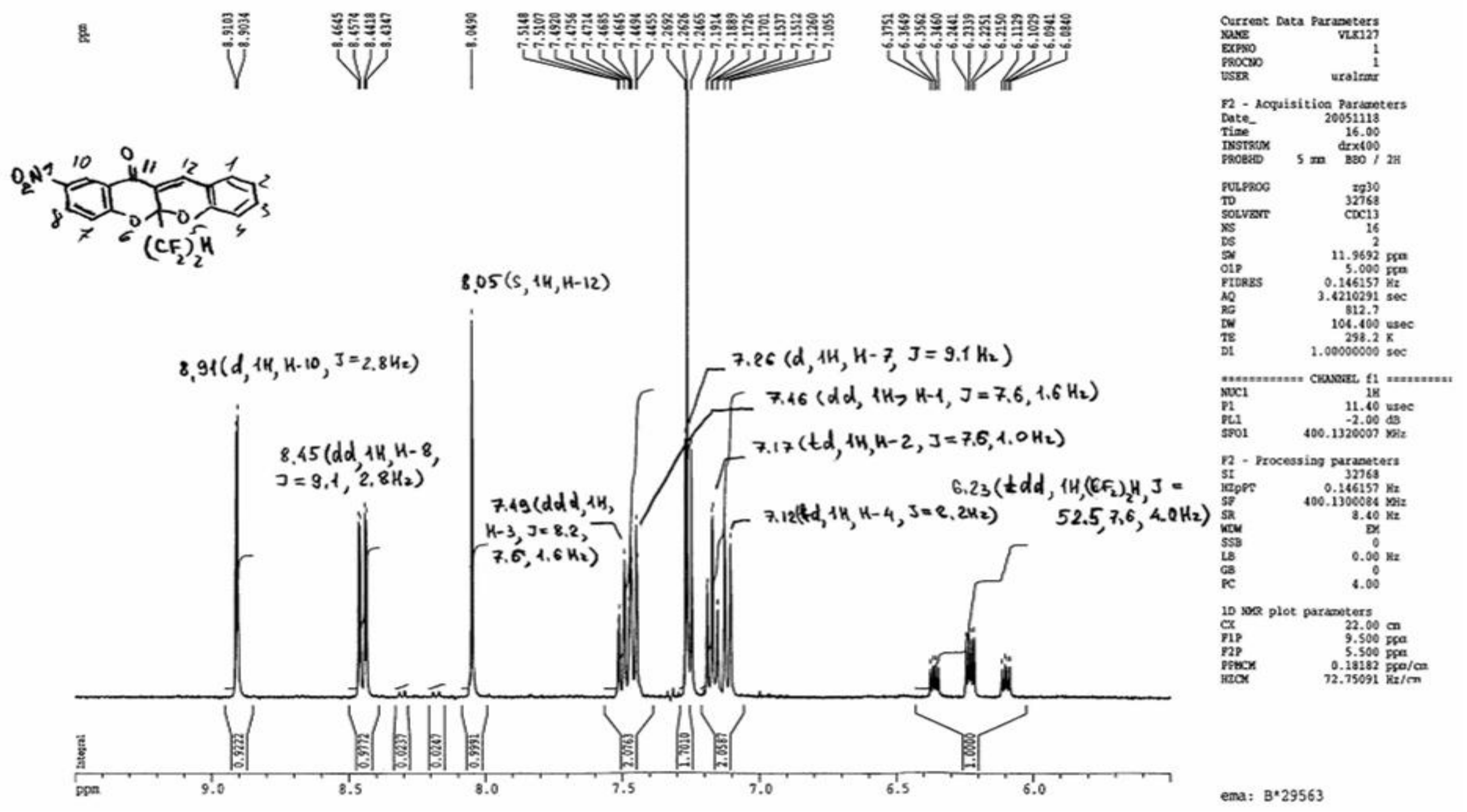


Copy of ${ }^{19}$ F NMR spectrum of $\mathbf{2 r}$
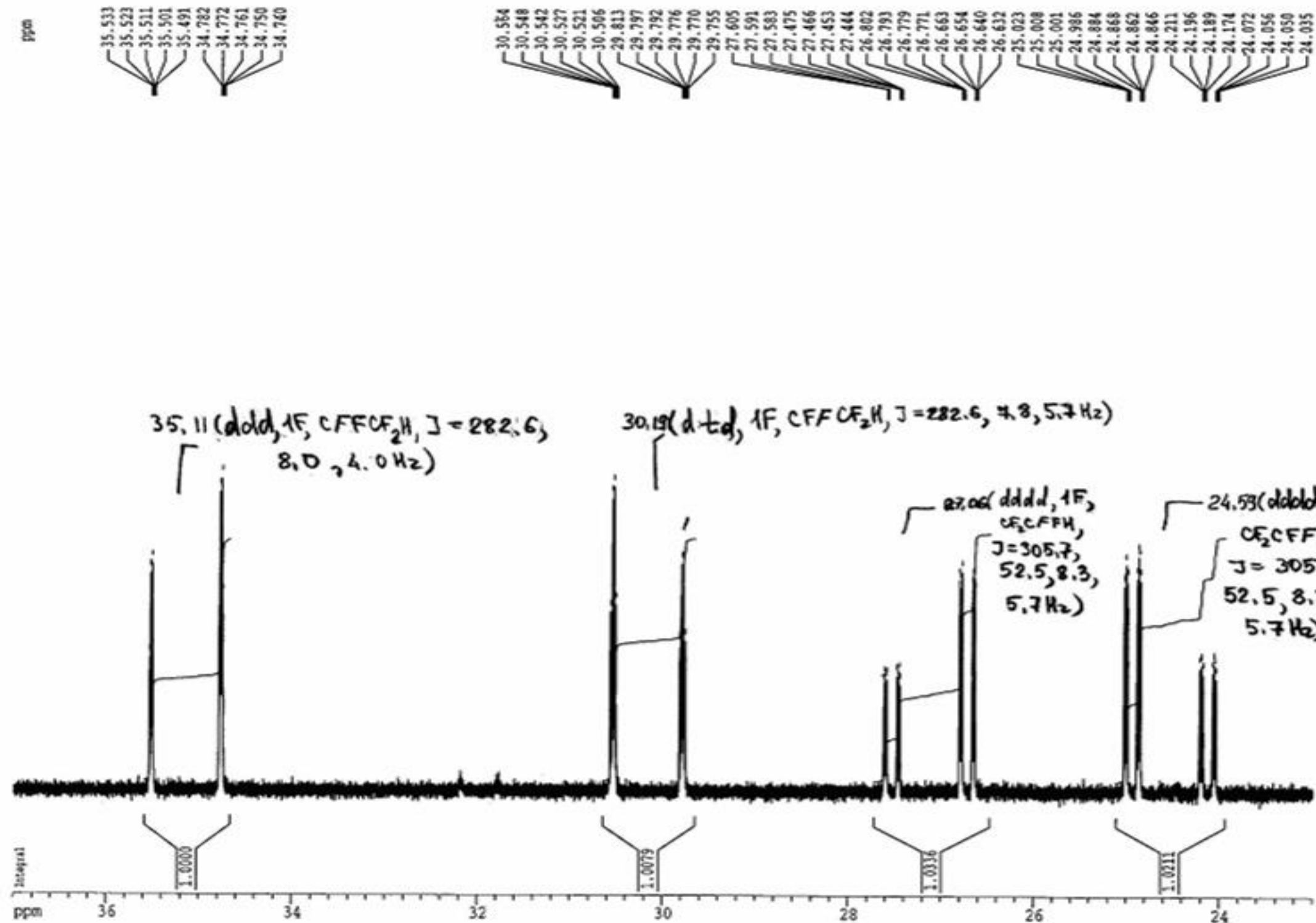

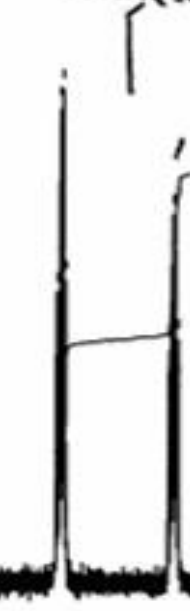

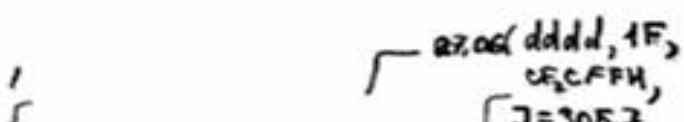
$J=305.7$,
$52.5,8.3$ $5.7 \mathrm{~Hz}_{2}$ ) $30.18(d t d$, , IF, CFFCF $\mathrm{H}, J=282.6,7.8,5.7 \mathrm{~Hz})$

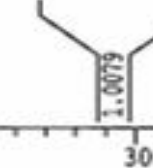

$\Gamma^{24.53(d d d d,}$, if $\left\{\begin{array}{l}C_{2} C F F H, \\ J=305,7, \\ 52,5,8,3,\end{array}\right.$ $5.7 \mathrm{He})$

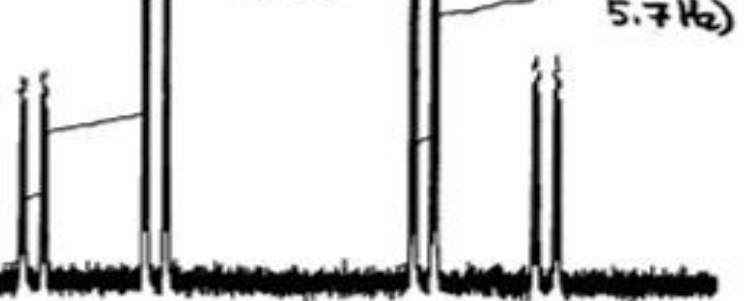

${ }^{10}$

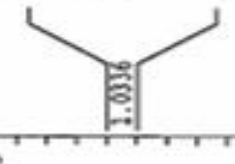

28
Current Data Paraneters

$\operatorname{saxg}$

$\operatorname{Paccos}$

USER

vixi27

urainerr

n2 - גcquisition paraneters

Date- 20051124

12.45
Tinstrax

m $380 / 2 \mathrm{H}$

Dutrance

socven:

is

OI?

FTtres

RQ

RG

Ts

2930
131072

Cocis$$
59.8295 \mathrm{pro}
$$

20.000 pro $0.171833 \mathrm{~Hz}$ 2.9098485 ste 22.200 usec $1.00000000 \mathrm{sec}$

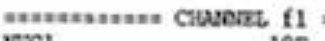

nocl $19 \%$

D1 11.90 usec

SP01 $\quad 376.4468137$ XEis

92 - Processing paraseters

SI - Processing paradeters

$\underset{S P}{0.171833} \mathrm{Kz}$

SR $201.26 \mathrm{~Hz}$

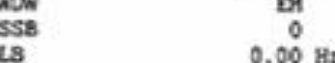

PC

$0.00 \mathrm{~Hz}$
2.00

10 BEQ plot paraceters

cx $\quad 22.00 \mathrm{~cm}$

$72 \% \quad 23.000 \mathrm{pon}$

PFAcr $\quad 0.63636 \mathrm{pgan} / \mathrm{cm}$

$239.55113 \mathrm{kz} / \mathrm{cm}$ 
Copy of ${ }^{1} \mathrm{H}$ NMR spectrum of $\mathbf{2 t}$

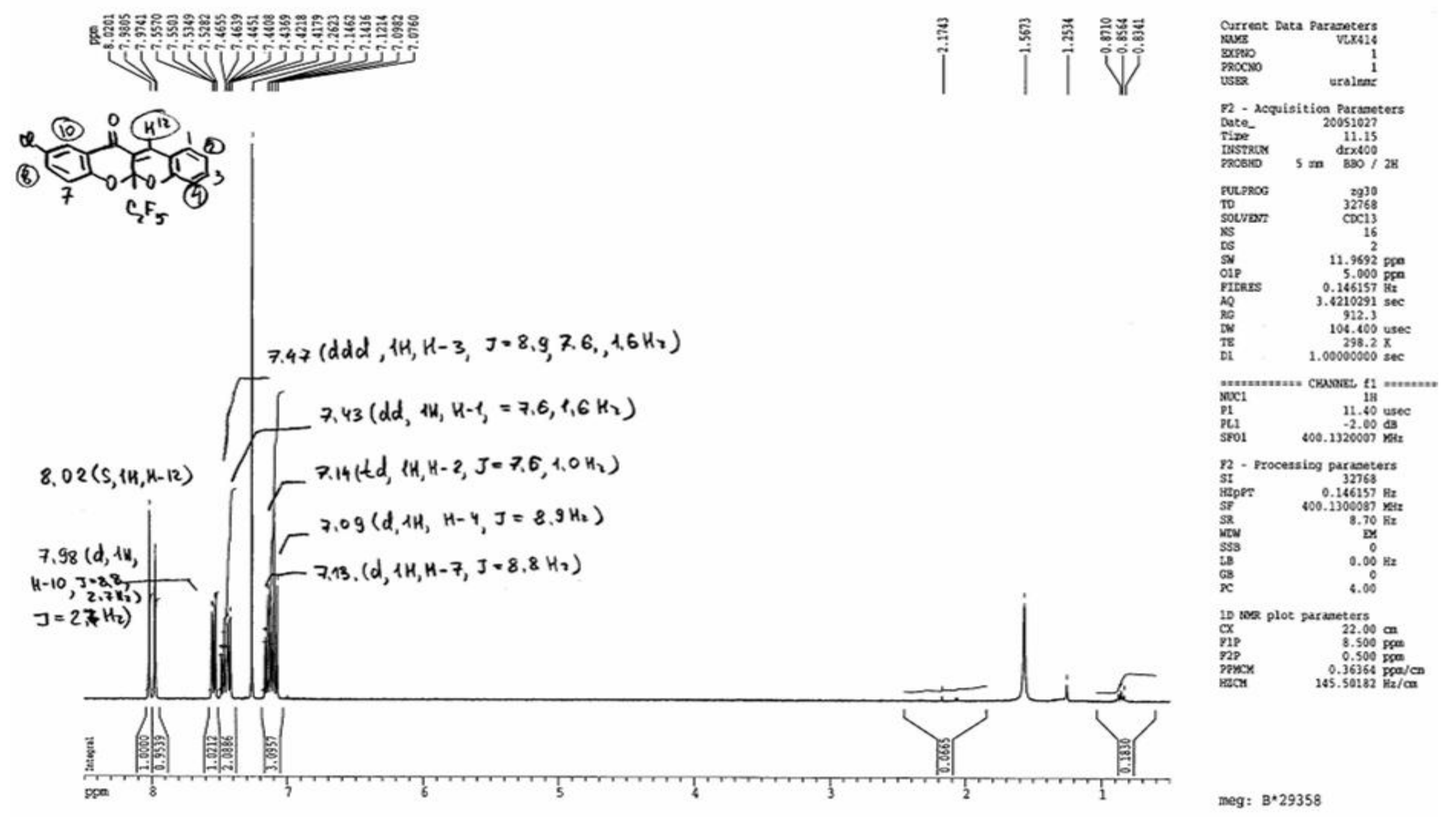




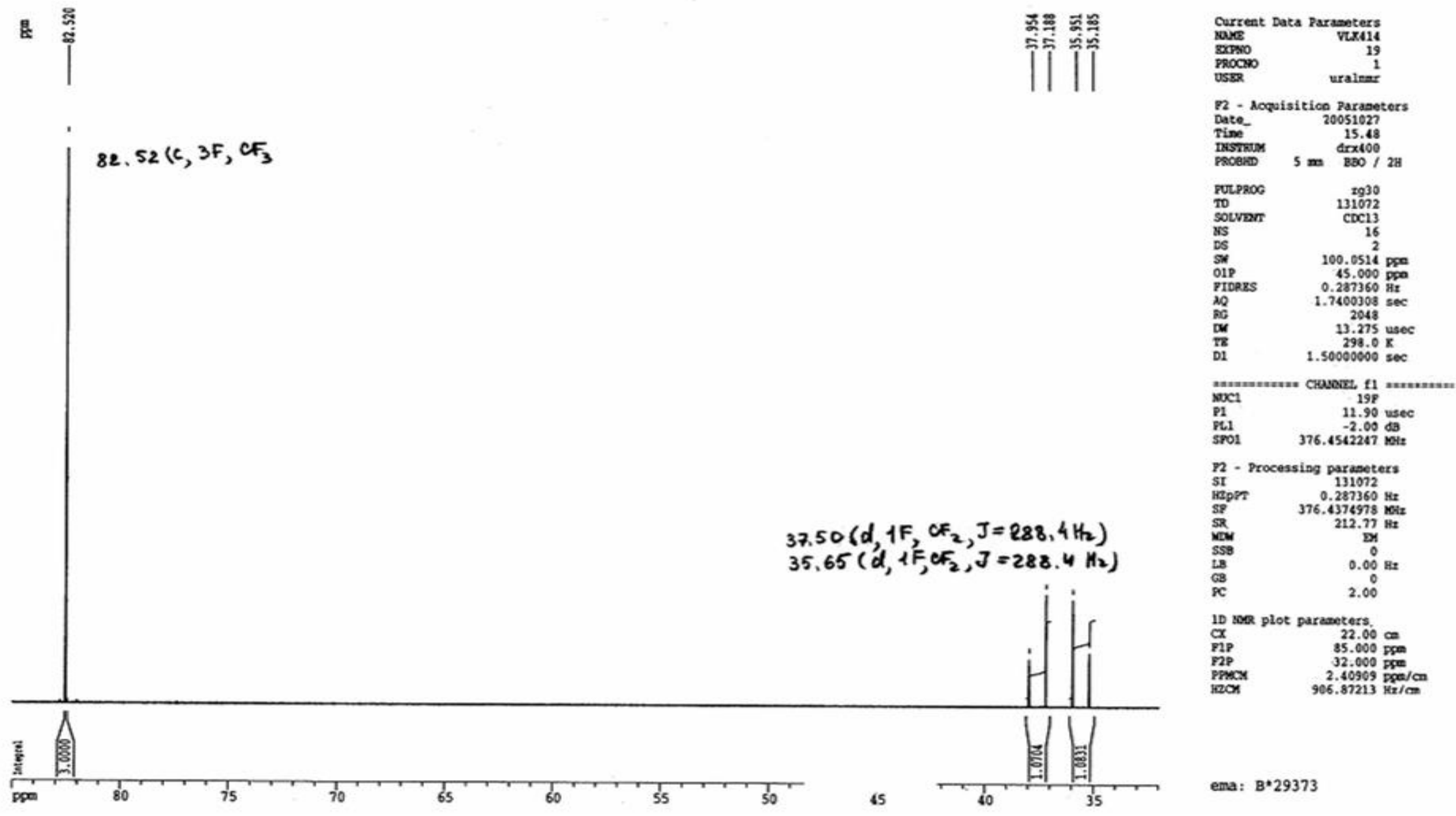




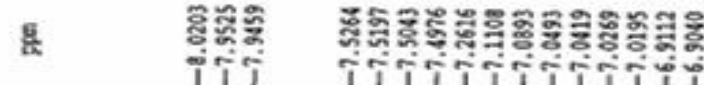

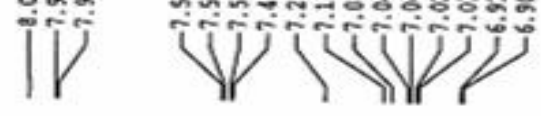

$v$
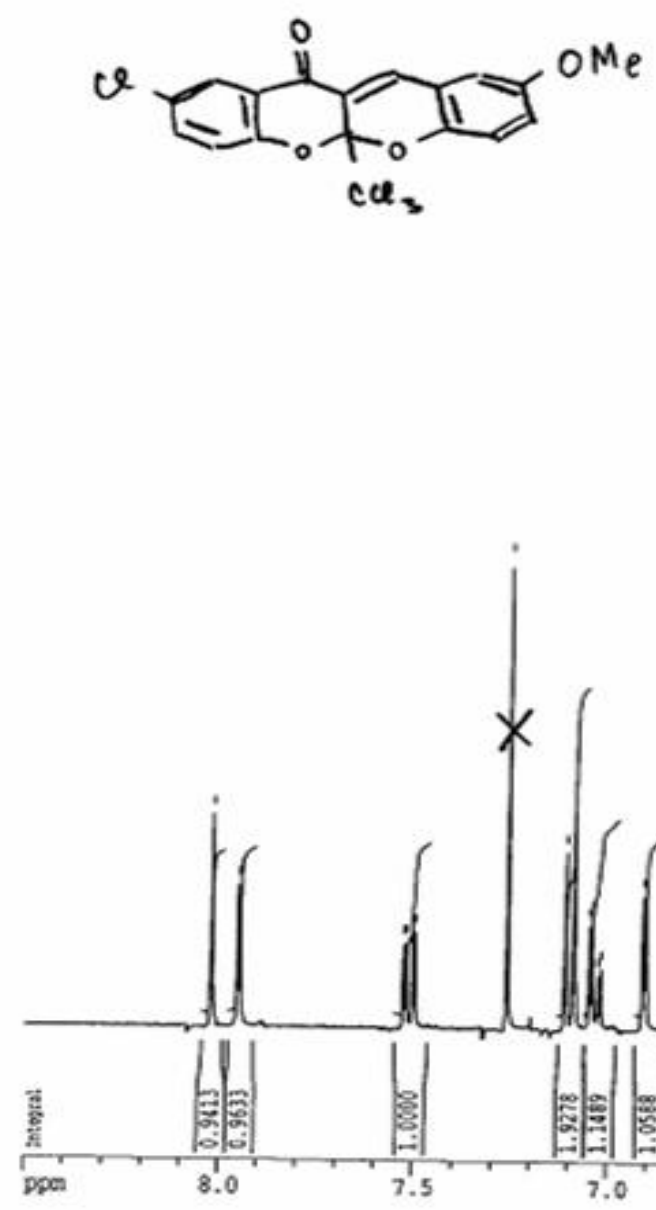
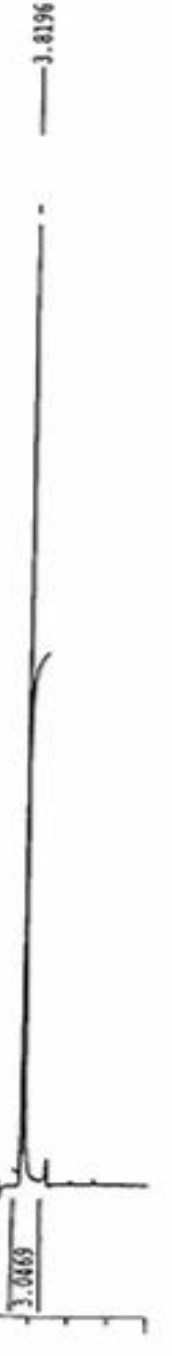

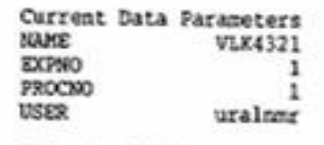

72 - Mcquisition paraseters

Date_- 20051130

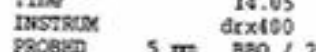

priproc

7D 30 trats

Sotvatr

DS

O1P

YroR

10
CW

Ts

$z g 30$

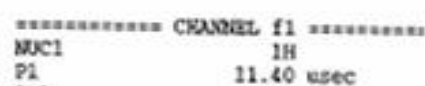

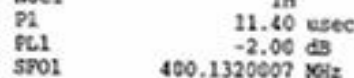

n2 - Processing parazeters

$\begin{array}{lll}\mathrm{SI} & 32768 \\ \mathrm{EP} & 0.146157 \mathrm{Ft} & \mathrm{Hz}\end{array}$

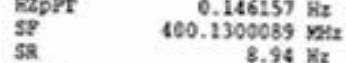

ister

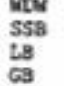

$8.94 \mathrm{Kz}$

54
$0.00 \mathrm{~Hz}$

4.00

10 WR plot paraheters

CX $\quad 22.00 \mathrm{~cm}$

Paper $3.500 \mathrm{pgen}$

Pancr $\quad 0.22727 \mathrm{pan} / \mathrm{cm}$ 
Copy of ${ }^{1} \mathrm{H}$ NMR spectrum of $\mathbf{3 c}$
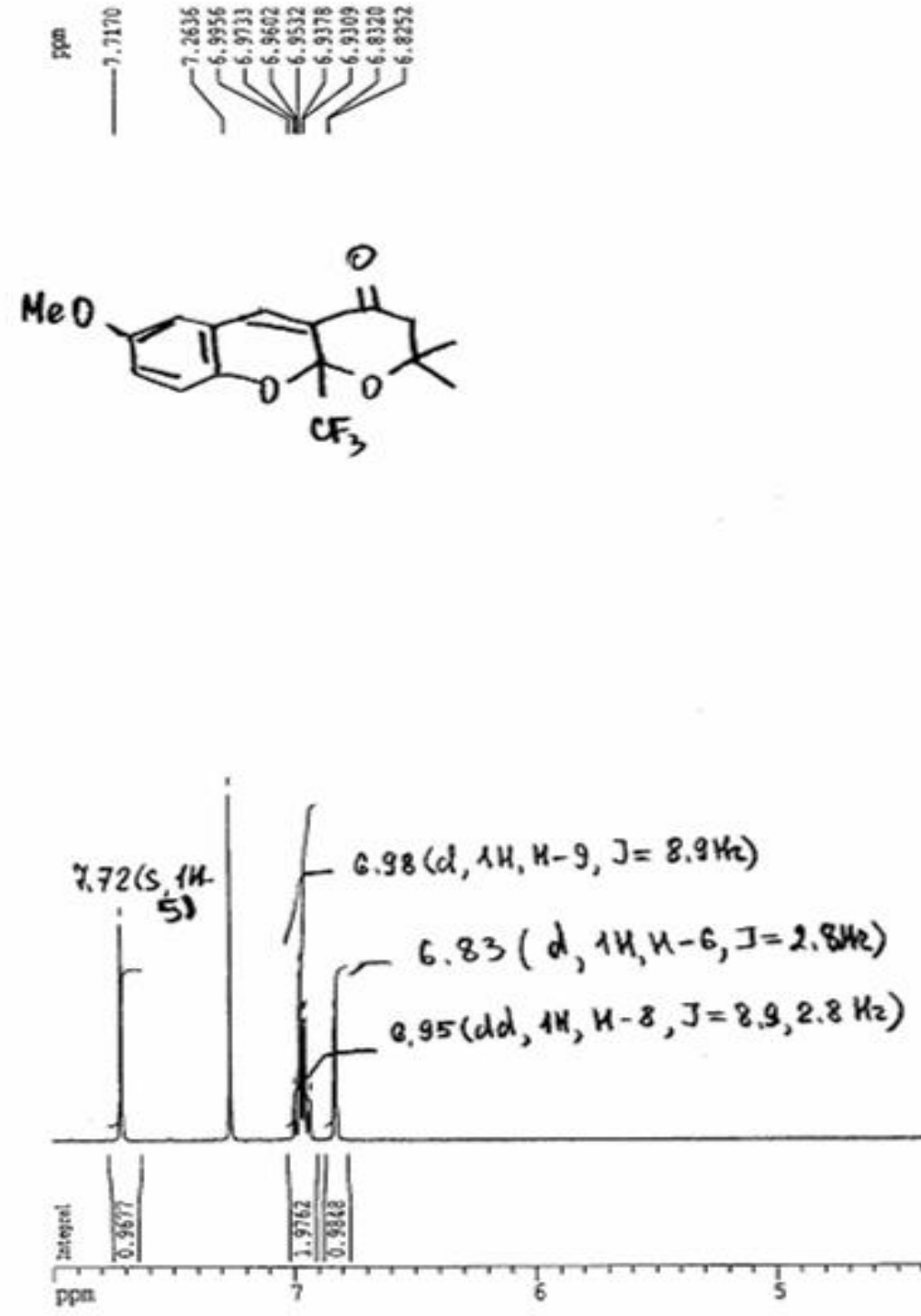

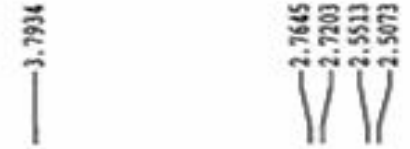

\section{$3.79(\mathrm{~S}, 3 \mathrm{H}, \mathrm{ONe})$}

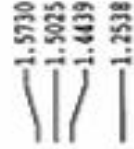

$2.53 \mathrm{Cd}, 1 \mathrm{H}, \mathrm{CH}$ $\left.J=17.6 \mathrm{H}_{2}\right)$ $2 . z_{4}(d, 14$, cul $k$, J=17.6Hz)

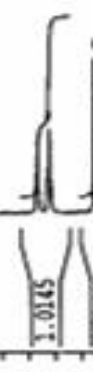

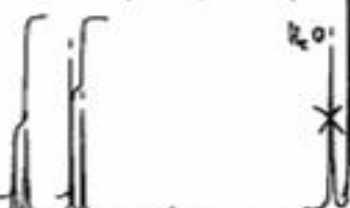

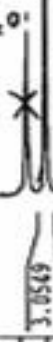

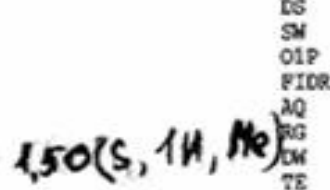

$1.4(5,3 H)$

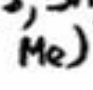

Me)

Di
Carrent Data Parageters

gxas

Proczos

(1)

72 - Acquisition Paramaters

Date: 20051123

7 ine 15.37

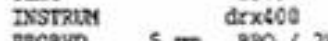

$850 / 2 \mathrm{H}$

PULPROC

Solvan

is

$10.0127 \mathrm{pgan}$ $0.122266 \mathrm{~Hz}$ $4.0896966 \mathrm{sec}$ 124.8120 utoe 124.800 ustoc $1.00000000 \mathrm{sec}^{\circ}$

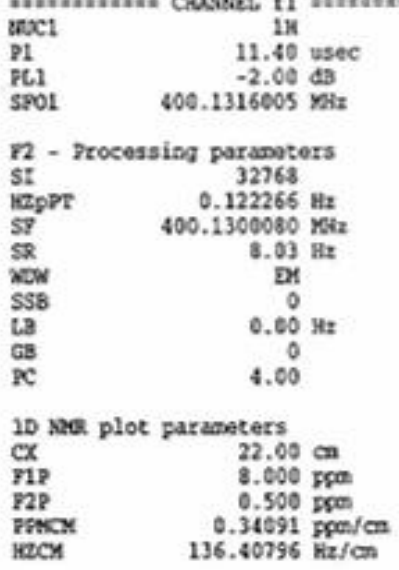

meg: B*29606 

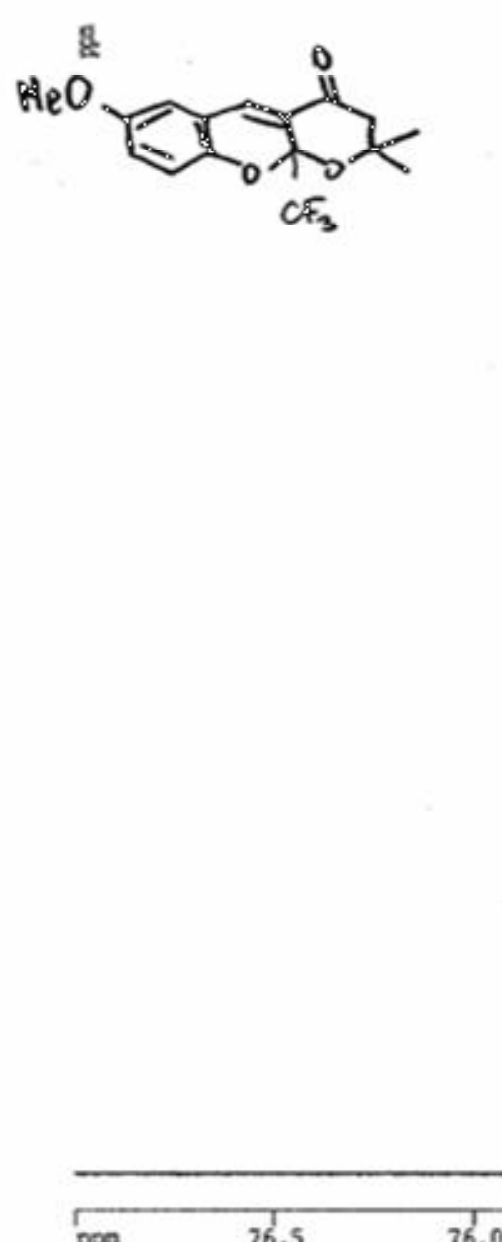

$\exists$

$74,64\left(5,81, \mathrm{CE}_{3}\right)$

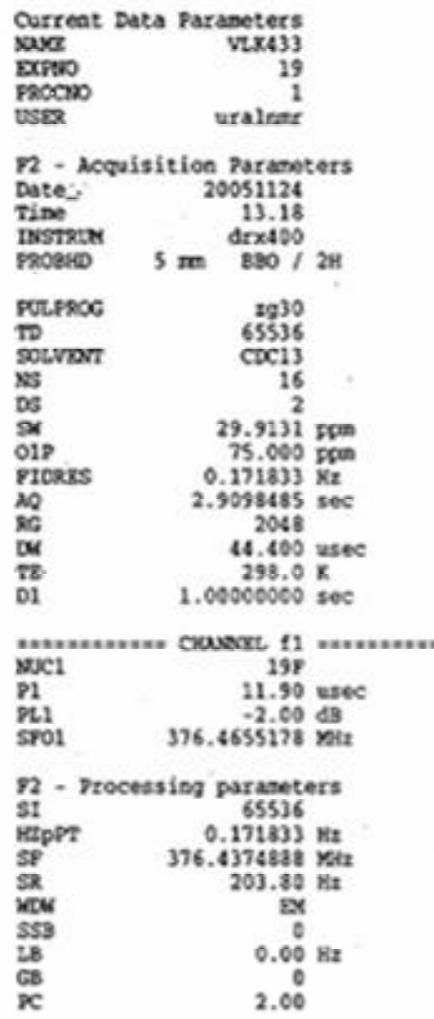

10 sa plot paranetet:

a $22.00 \mathrm{~cm}$

$728 \quad 72.000 \mathrm{pgec}$

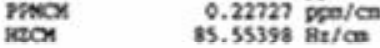


Copy of ${ }^{13} \mathrm{C}$ NMR spectrum of $\mathbf{3 c}$

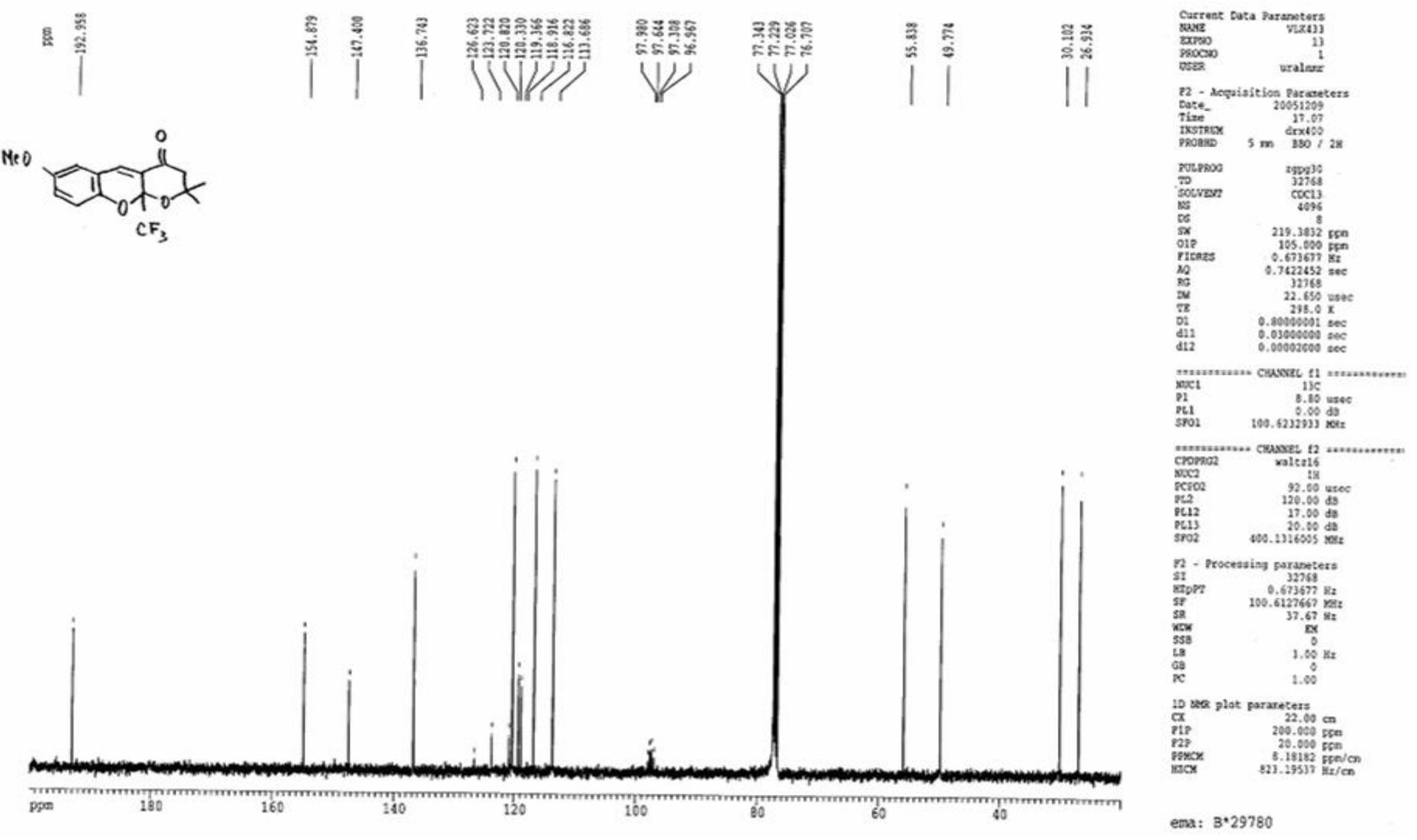

PDF created with FinePrint pdfFactory Pro trial version www.pdffactory.com 
Copy of ${ }^{1} \mathrm{H}$ NMR spectrum of $\mathbf{3 d}$

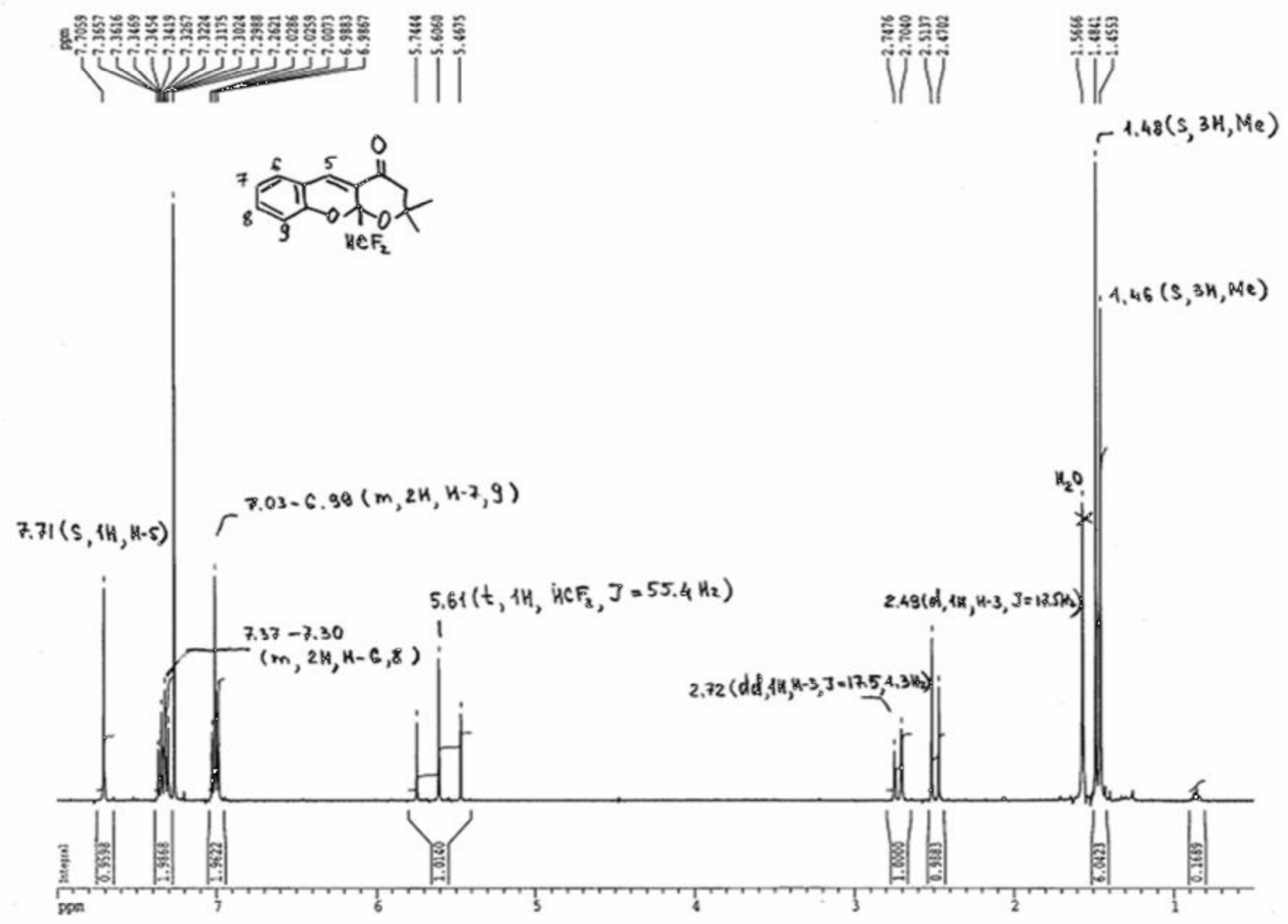


Copy of ${ }^{19}$ F NMR spectrum of $\mathbf{3 d}$

量

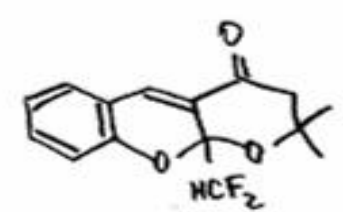

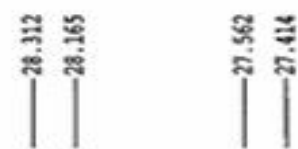

|

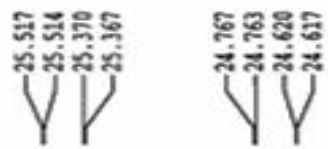

25,12 (ddd, $1 F, \mathrm{MF}_{2}, J=282,4,55,2,1.3 \mathrm{~Hz}$ )

$27.81\left(d d_{1} 1 F, H C F_{2}, J=282.4,55.6 \mathrm{~Hz}\right)$

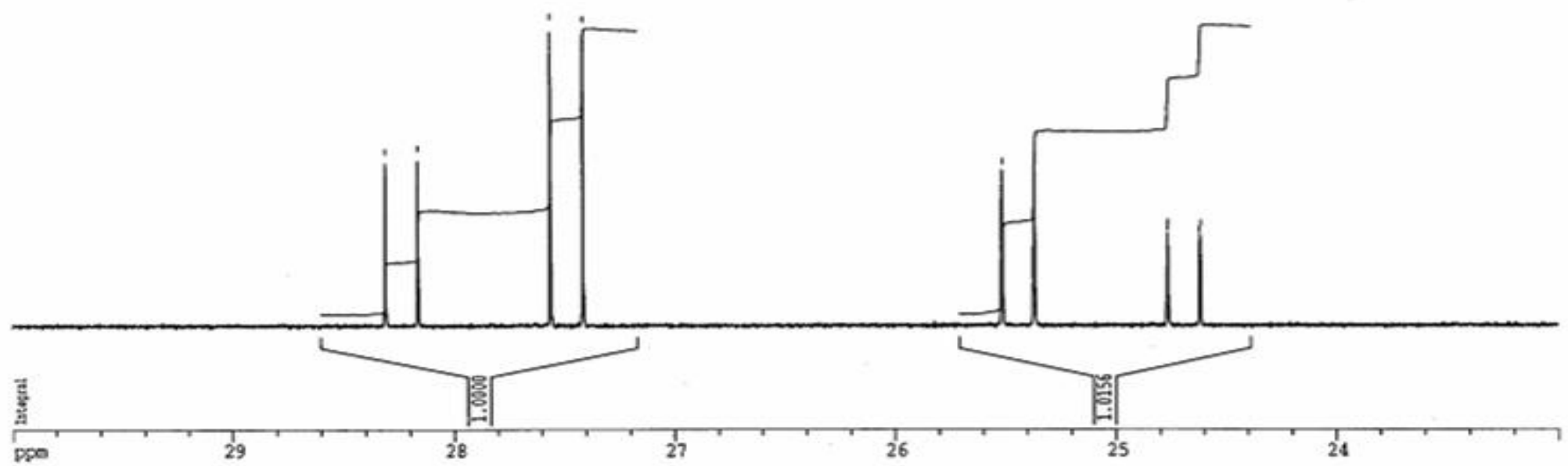

Current Data Parabeters

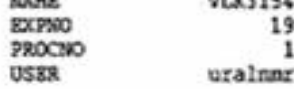

n2 - Acquisition paraneters
Date 20051018

Tioe- 14.17

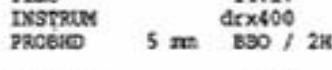

PULPROC

Soctran:

solvar
is
ss

OIP

FIros:

MQ

TIS

o1

sg30

$\mathrm{CDC13}$

$40.0067^{2}$

15.000 pro $0.229301 \mathrm{~Hz}$ 2.175851300 33.200 usec $1.00000000 \mathrm{sec}$

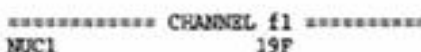

P1 $1199 \mathrm{usec}$

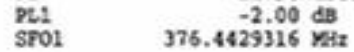

92 - Processing parameters

$\begin{array}{ll}\text { SI } & 65536 \mathrm{Ft} \\ \text { Hapt } & 0.229801 \mathrm{~Hz}\end{array}$

RapPT
SP

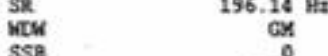

$\begin{array}{lc}\text { SSB } & 0.0 \\ \text { LS } & -0.50^{\circ} \mathrm{Hz} \\ G \mathrm{~B} & 0.25\end{array}$

2.00

10 BQR plot paraneters $22.00 \mathrm{co}$

F1P $\quad 30.000 \mathrm{pge}$

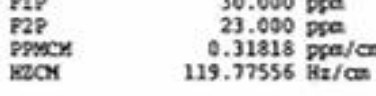

ema: $B * 29282$ 
Copy of ${ }^{1} \mathrm{H}$ NMR spectrum of $\mathbf{3 f}$
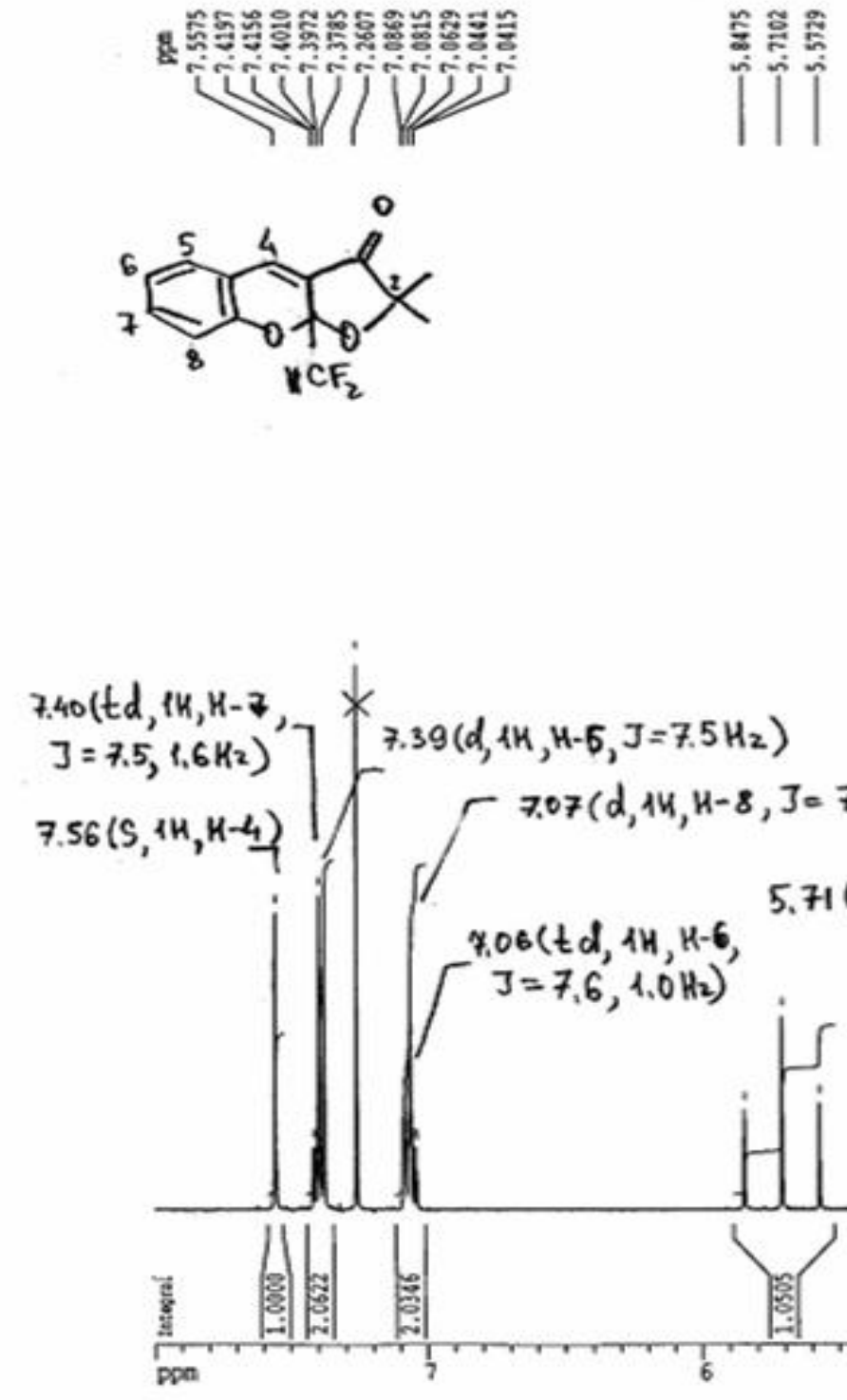

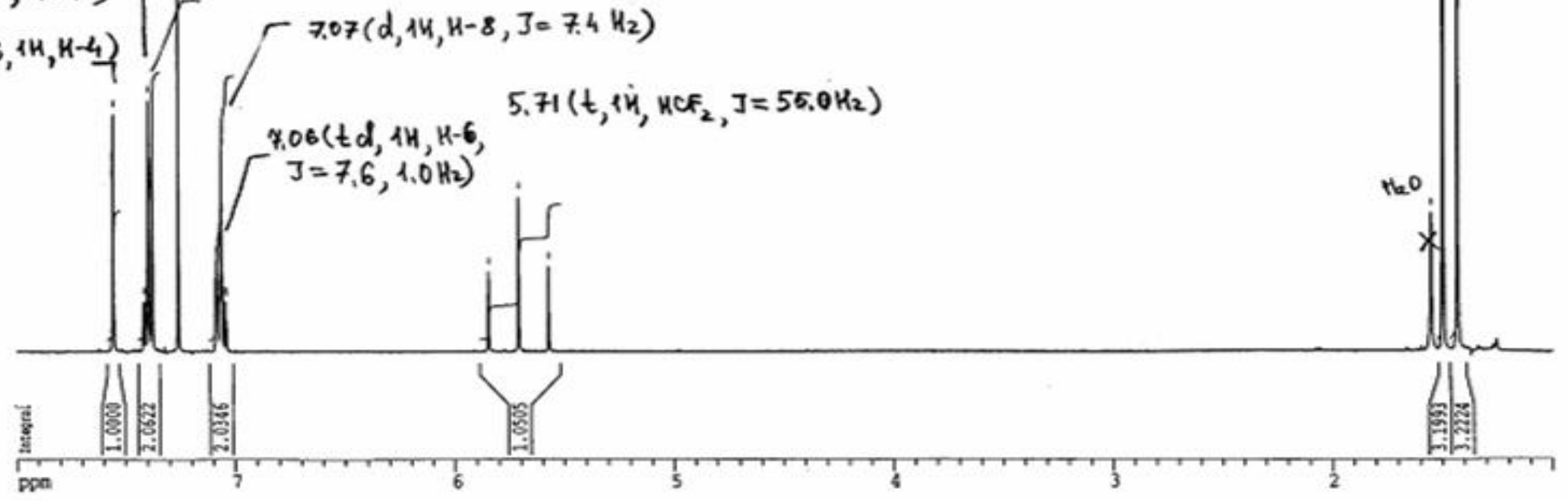

Curreat Data Paraneters

vavg vix319

Exraso

toses

uralous

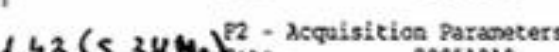

hate_ acquisition Parader 20051018

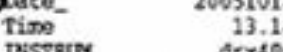

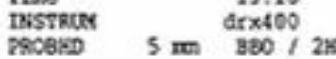

TULPROG $\quad$ sg30

32768
solvant
cocis

DS

\$1 $\quad 11.9692$ prom

PIDRES $\quad 0.26157 \mathrm{~Hz}$

2Q $\quad 3.4210291 \mathrm{sec}$

tra

104.400 usec

$298.2 \mathrm{x}$

D1 $1.00000000 \mathrm{sec}$

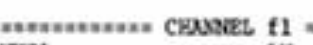

nxc1 11.40 usec

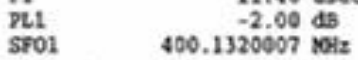

72 - processing paramoters

SI Tocessing parrostors

RIpP? $\quad 0.146157 \mathrm{kz}$

SF $\quad 400.1300093 \mathrm{xet}$

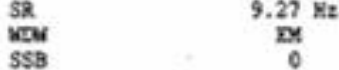

${ }_{\mathrm{LB}}^{S S B} \quad 0.00 \mathrm{~Hz}$

1D 12 p plot paraseters

cx $22.00 \mathrm{~cm}$

$18 \mathrm{pos}$

Frack $0.31813 \mathrm{pros} / \mathrm{Cm}$

ema: $\mathbf{8 * 2 9 2 7 6}$ 
Copy of ${ }^{19}$ F NMR spectrum of $\mathbf{3 f}$

岳

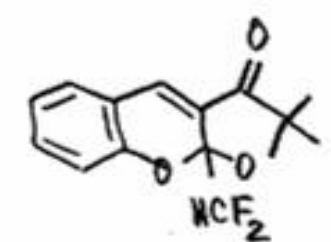

$28.93\left(d d, 1 F_{1} u \in E_{2}, J=287.6,55.1 \mathrm{~Hz}\right)$$$
\text { 를 }
$$
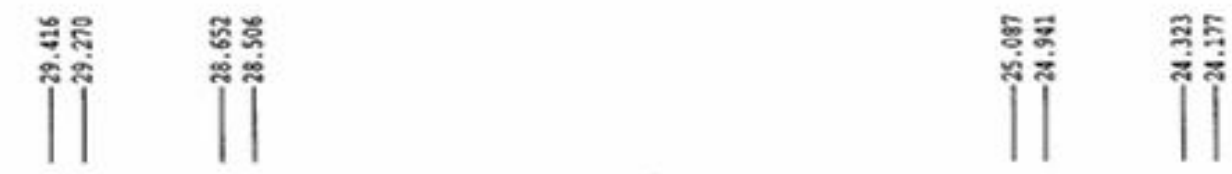

$\mathrm{NCF}_{2}$

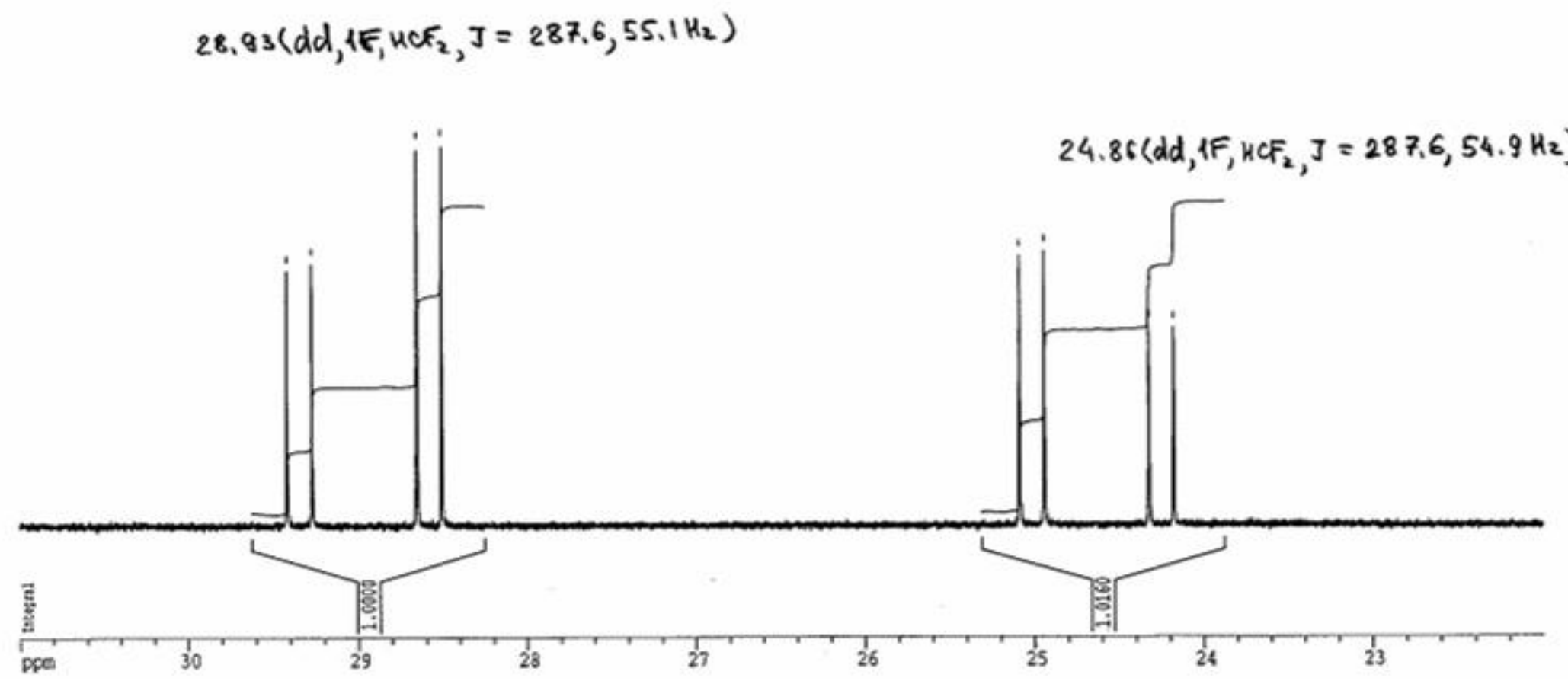

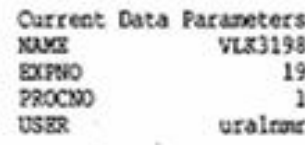

72 - Acyuisition Pazasoters

Date_ 20051018

TSSTRIM dex600

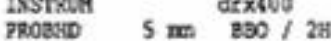

PULPRCC

TD

sotvern

is

24

OIP

Prores

89

tow

$\mathrm{TS}$
$\mathrm{D} 1$

metis

${ }_{21}$

Ri
spot

srot

2 - Processieg pareanoters

65536

gept $0.229801 \mathrm{dz}$

$376.4374302 \mathrm{Xt}$
$195.22 \mathrm{~Hz}$

KDW

$\mathrm{CSB}_{\mathrm{La}} \quad 0.00^{\circ} \mathrm{Hz}$

$\stackrel{2.00}{\oplus}$

ID teR plot paraseters

cx 22.000

$917 \quad 31.000 \mathrm{pgat}$

PIP $22.000 \mathrm{pme}$

rack $\quad 153.99715 \mathrm{kz} / \mathrm{cm}$

exa: B.2928 
Copy of ${ }^{1} \mathrm{H}$ NMR spectrum of trans-6a

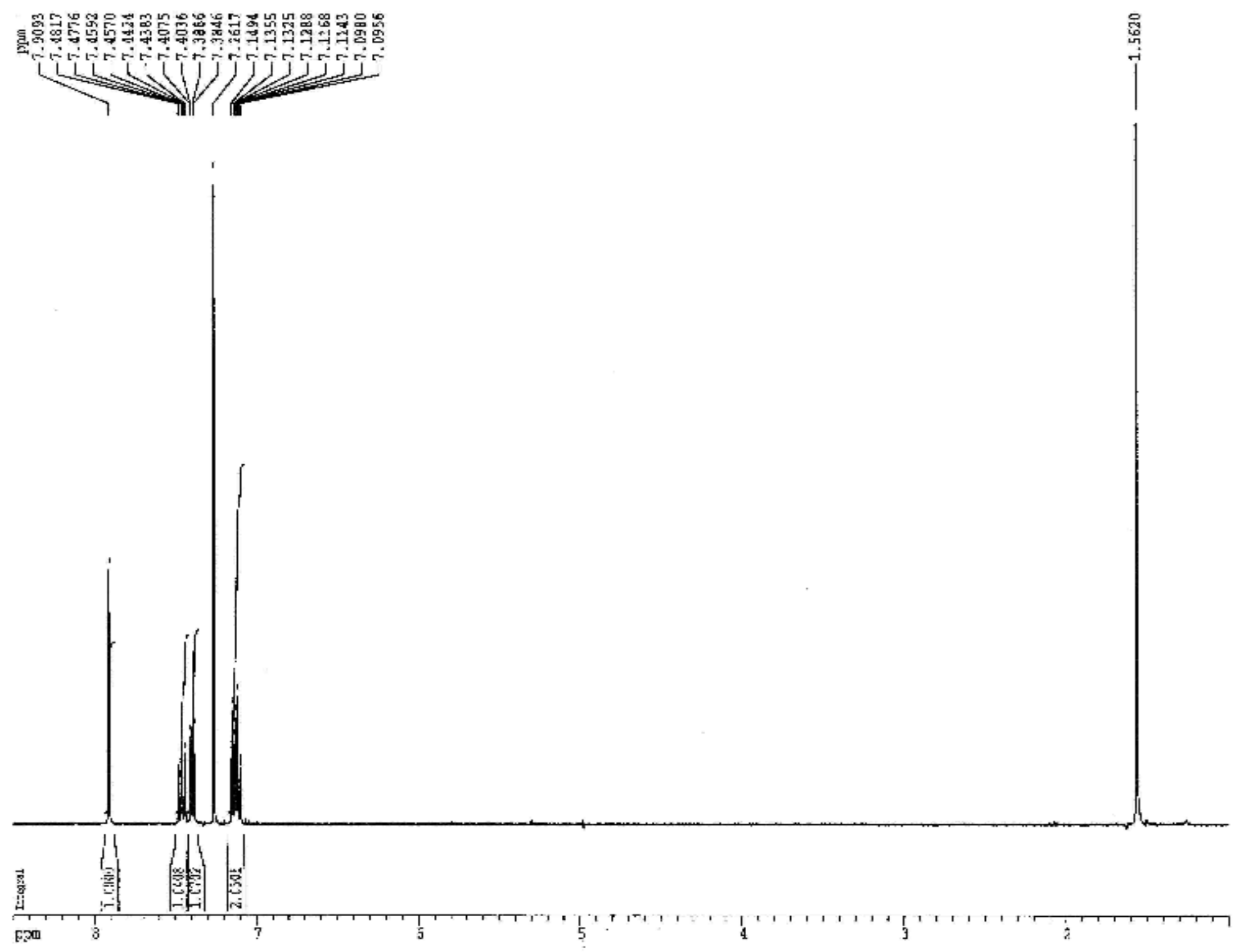

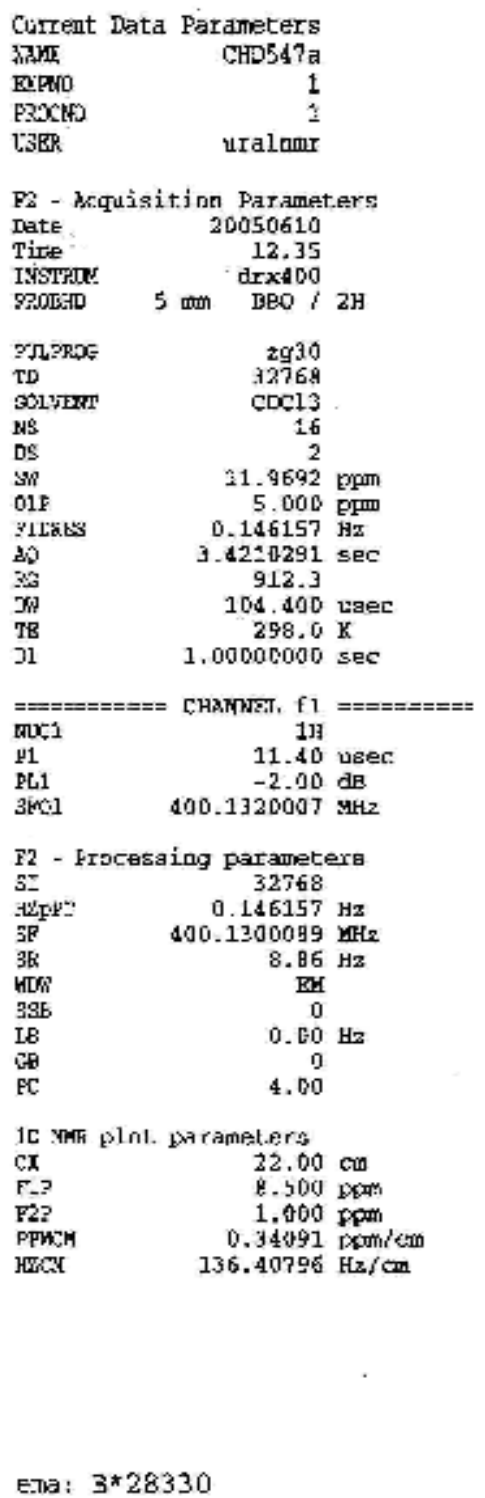


Copy of ${ }^{13} \mathrm{C}$ NMR spectrum of trans-6a

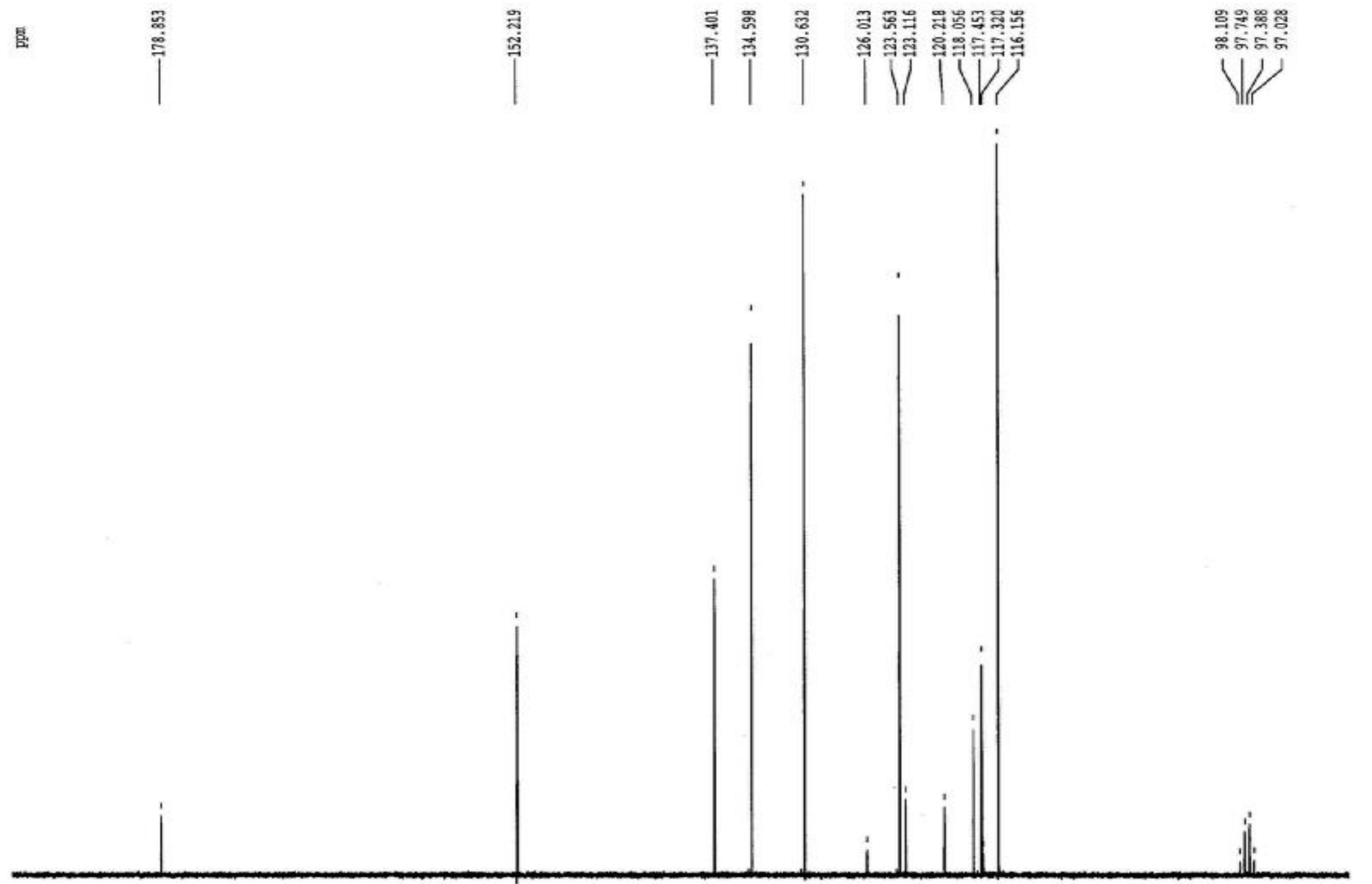

ppm

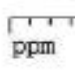

180

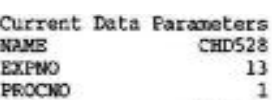

USER

F2 - Acçuisition Parameters

Date- 20050525

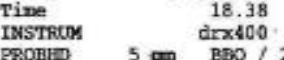

PULPROG
Tgpg 30
65536

$\begin{array}{ll}\text { TD } & 65536 \\ \text { SOLIVENT } & \text { CPCC13 } \\ \text { NS } & 18432 \\ \text { PS } & \text { S }\end{array}$

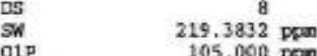

TERES $\quad 0.336839 \mathrm{~Hz}$

RQ $\quad 1.4844404 \mathrm{sec}$

22.550 use
$298.0 \mathrm{~K}$

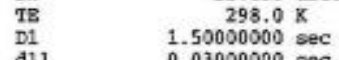

$\begin{array}{ll}\mathrm{D} 1 & 1.50000000 \mathrm{sec} \\ \mathrm{d} 11 & 0.03000000 \\ \mathrm{~d} 12 & 0.00002000\end{array}$

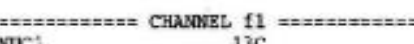

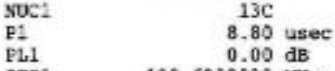

103.5232933

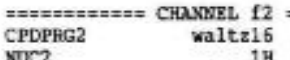

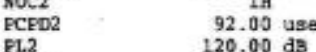

FL12 $\quad 120.00 \mathrm{~dB}$

PL13
SPO2

F2 - Processing paraneters

I $\quad 65536$

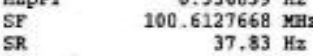

$\begin{array}{ll}S R & 37.83 \\ \text { WTM } & -9.5 \%\end{array}$

$\begin{array}{ll}\mathrm{SSB} & 0 \\ \mathrm{LB} & -0.50 \mathrm{~Hz} \\ \mathrm{~GB} & 0.25\end{array}$

ID RR plot paraneters

22.00 a

${ }_{22 \mathrm{P}}^{\mathrm{F}}$

PreMCX
HECK

$96.600 \mathrm{ppm}$ $4.54515 \mathrm{ppn} / \mathrm{cm}$
$457,33678 \mathrm{Ez} / \mathrm{cm}$

ema: $B^{\star} 2815$ 
Copy of ${ }^{1} \mathrm{H}$ NMR spectrum of $c i s-6 \mathbf{a}$

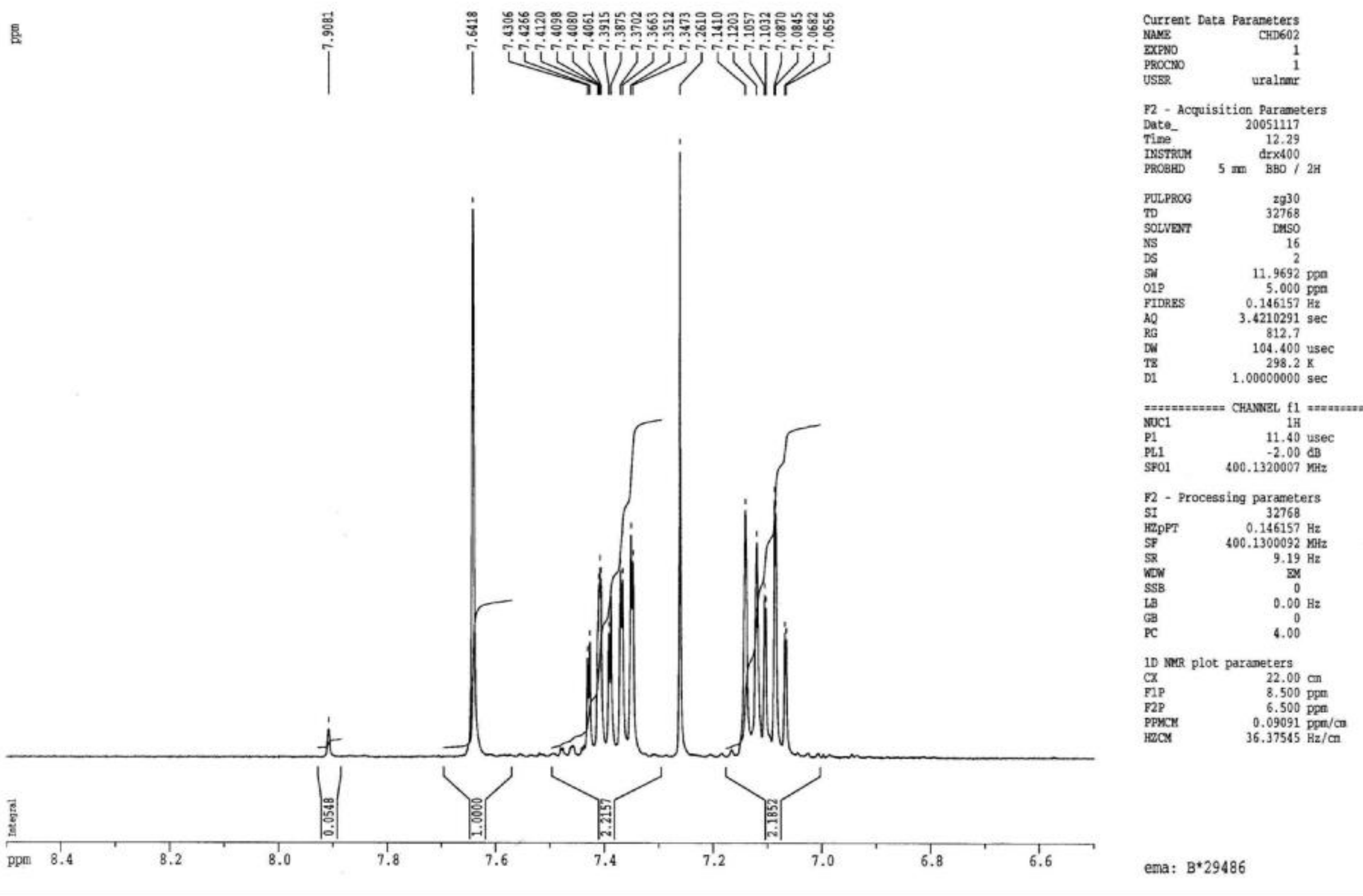




\section{Copy of ${ }^{1} \mathrm{H}$ NMR spectrum of $7 \mathbf{a}$}

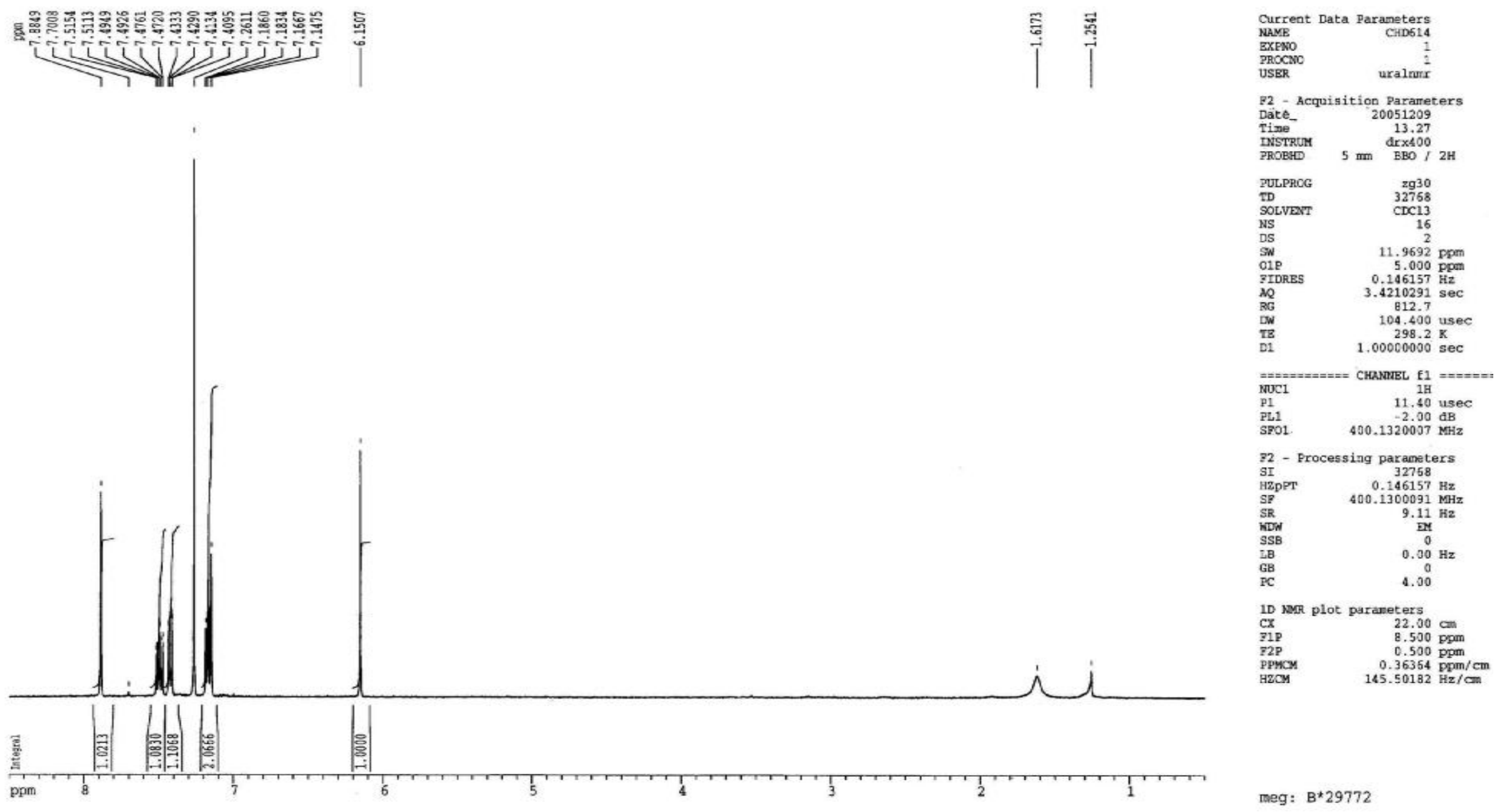


Copy of ${ }^{1} \mathrm{H}$ NMR spectrum of $7 \mathrm{c}$
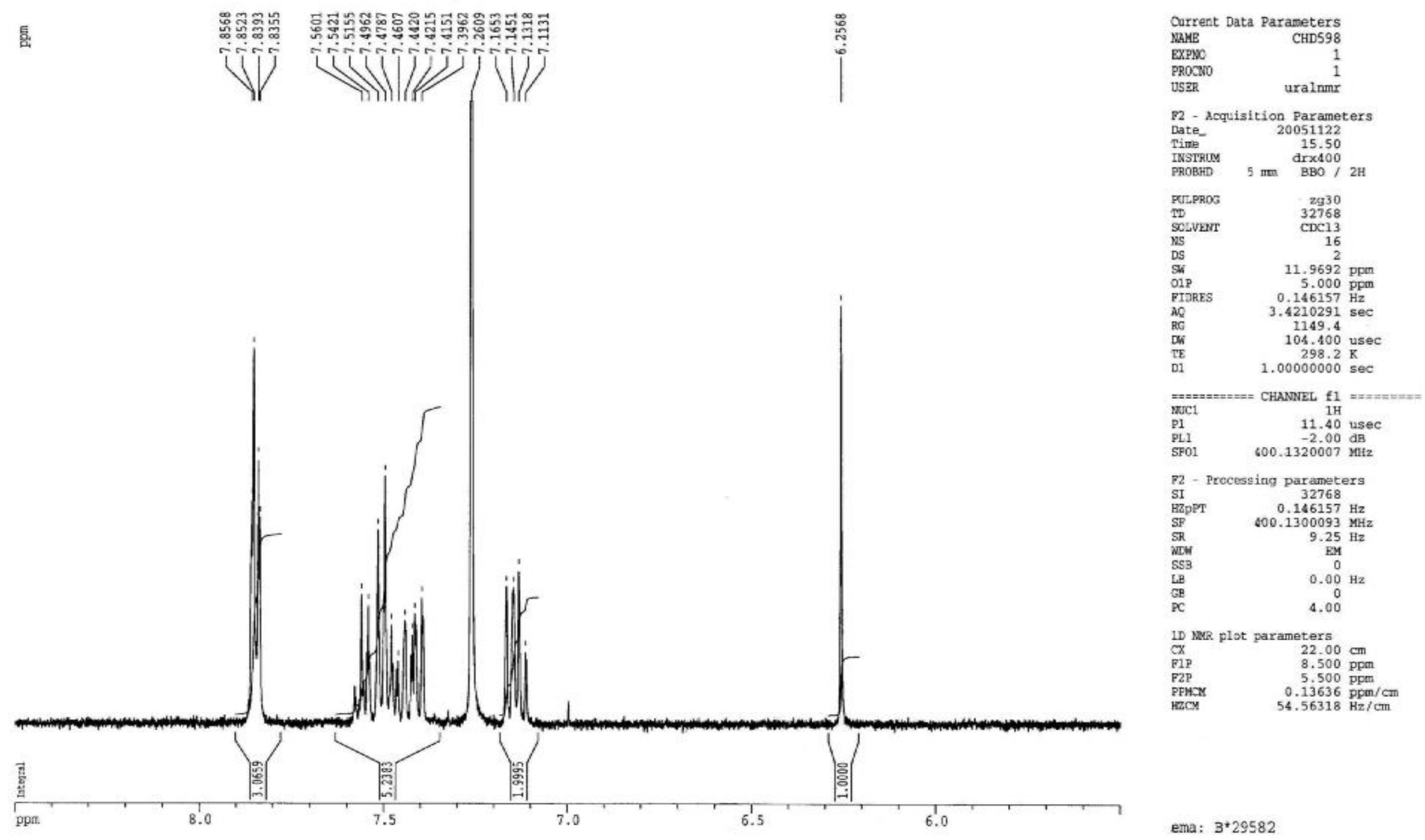